\title{
Cyclic Ureate Tantalum Catalyst for Preferential Hydroaminoalkylation with Aliphatic Amines: Mechanistic Insights into Substrate Controlled Reactivity
}

Pargol Daneshmand†, Sorin-Claudiu Roşca†, Rosalie Dalhoff $†$, Kejun Yin†, Rebecca C.

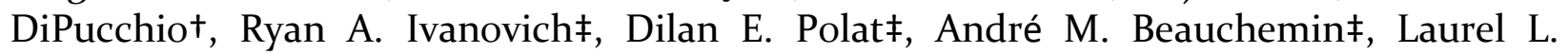
Schafer ${ }^{*}+$

+ Department of Chemistry, University of British Columbia, 2036 Main Mall, Vancouver, B.C, Canada, V6T 1 Z1.

¥ Centre for Catalysis Research and Innovation, Department of Chemistry and Biomolecular Sciences, University of Ottawa, 10 Marie-Curie, Ottawa, ON K1N 6N5, Canada.

S2. Instrumentation

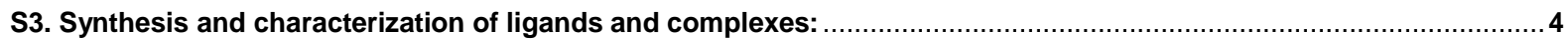

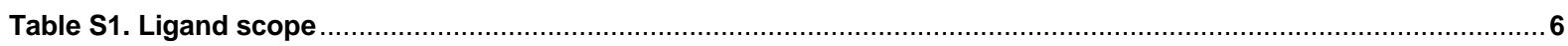

Table S2. Solid- $G$ parameters $G \mathrm{~L}$ and $G_{\text {space }}$ calculated for ureate tantalum catalysts .................................................. 7

Figure S1. Solid-G output for complex 1 (top) and 1a (bottom). Percentage of occupancy by ureate ligand shown in yellow.....7

Figure S2. Conversion vs time plot observed for the hydroaminoalkylation reaction of $\mathrm{Ta}\left(\mathrm{CH}_{2} \mathrm{SiMe}_{3}\right)_{3} \mathrm{Cl}{ }_{2}+\mathrm{L} 2 \mathrm{Na}$ (in situ) or $\mathbf{1}$ with $\mathrm{N}$ -

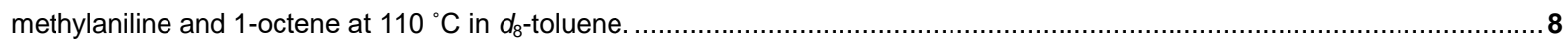

Figure S3. Comparison of $k_{\text {obs }}$ values observed for the hydroaminoalkylation reaction of $\mathrm{Ta}\left(\mathrm{CH}_{2} \mathrm{SiMe}_{3}\right)_{3} \mathrm{Cl}_{2}+\mathbf{L 2} \mathrm{Na}$ (in situ) or $\mathbf{1}$ with $\mathrm{N}$-methylaniline and 1 -octene at $110{ }^{\circ} \mathrm{C}$ in $d_{8}$-toluene.

Figure S4. Conversion vs time plot observed for $0.5 \mathrm{mmol} \mathrm{N}$-methylcyclohexylamine (diamonds), $0.5 \mathrm{mmol} N$-methylaniline (circles), $0.5 \mathrm{mmol} 1$-octene and $5 \mathrm{~mol} \% 1$ in $0.5 \mathrm{~mL}$ of $d_{8}$-toluene at $105{ }^{\circ} \mathrm{C}$.

9

Figure S5. Comparison of $k_{\mathrm{obs}}$ values observed for $0.5 \mathrm{mmol} \mathrm{N}$-methylcyclohexylamine (diamonds), $0.5 \mathrm{mmol} N$-methylaniline

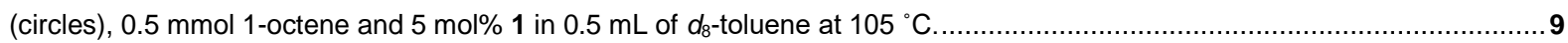

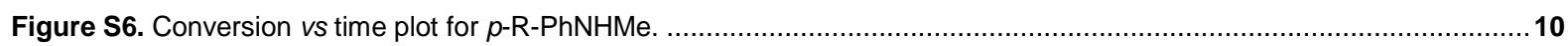

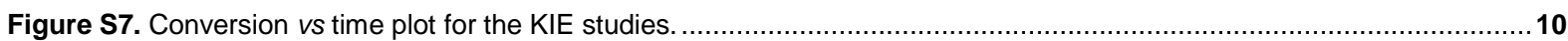

Figure S8. Hammett plot for the reaction of $p$-R-PhNHMe where $\mathrm{R}=\mathrm{OMe}, \mathrm{Me}, \mathrm{H}, \mathrm{F}, \mathrm{Cl}$ and $\mathrm{Br}$.....................................11

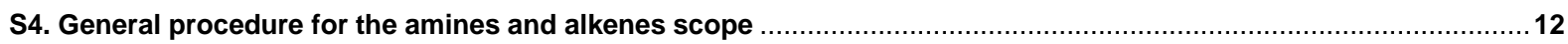

Figure S9. ${ }^{1} \mathrm{H}$ NMR spectrum $\left(300 \mathrm{MHz}, \mathrm{CDCl}_{3}, 298 \mathrm{~K}\right)$ of 1 -(tert-butyl)imidazolidin-2-one (L2H)........................................15

Figure $\mathbf{S 1 0 .}{ }^{13} \mathrm{C}$ NMR spectrum $\left(75 \mathrm{MHz}, \mathrm{CDCl}_{3}, 298 \mathrm{~K}\right)$ of 1-(tert-butyl)imidazolidin-2-one (L2H)......................................15

Figure S11. ${ }^{1} \mathrm{H}$ NMR spectrum $\left(300 \mathrm{MHz}, \mathrm{CDCl}_{3}, 298 \mathrm{~K}\right)$ of 1 -cyclohexylimidazolidin-2-one (L3H)..................................... 16

Figure S12. ${ }^{13} \mathrm{C}$ NMR spectrum $\left(100 \mathrm{MHz}, \mathrm{CDCl}_{3}, 298 \mathrm{~K}\right)$ of 1-cyclohexylimidazolidin-2-one (L3H)...................................16

Figure S13. ${ }^{1} \mathrm{H}$ NMR spectrum $\left(300 \mathrm{MHz}, \mathrm{CDCl}_{3}, 298 \mathrm{~K}\right)$ of 1-phenylimidazolidin-2-one $(\mathrm{L} 4 \mathrm{H})$. ........................................17

Figure $514 .{ }^{13} \mathrm{C}$ NMR spectrum $\left(75 \mathrm{MHz}, \mathrm{CDCl}_{3}, 298 \mathrm{~K}\right)$ of 1-phenylimidazolidin-2-one $(\mathbf{L} 4 \mathrm{H})$.........................................17

Figure S15. ${ }^{1} \mathrm{H}$ NMR spectrum $\left(400 \mathrm{MHz}, \mathrm{CDCl}_{3}, 298 \mathrm{~K}\right)$ of 4,4-dimethyl-1-phenylimidazolidin-2-one (L5H) .........................18

Figure $516 .{ }^{13} \mathrm{C}$ NMR spectrum $\left(75 \mathrm{MHz}, \mathrm{CDCl}_{3}, 298 \mathrm{~K}\right)$ of 4,4-dimethyl-1-phenylimidazolidin-2-one (L5H).........................18

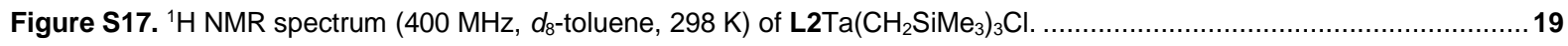

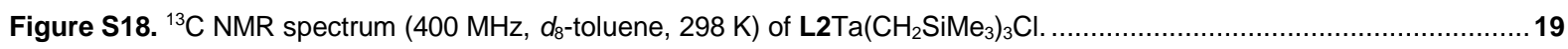

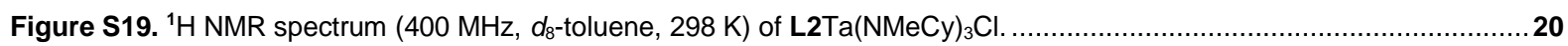

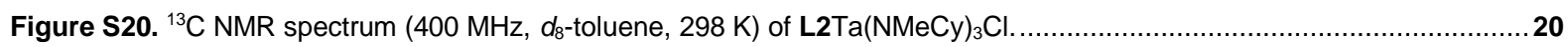

Figure S21. ${ }^{1} \mathrm{H}$ NMR spectrum $\left(400 \mathrm{MHz}, \mathrm{CDCl}_{3}, 298 \mathrm{~K}\right)$ of $\mathrm{N}$-(2-methyloctyl)cyclohexanamine (3) ...................................21

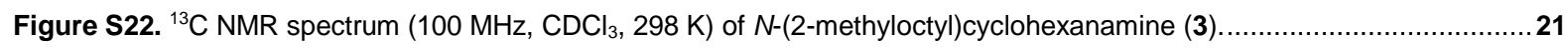

Figure S23. ${ }^{1} \mathrm{H}$ NMR spectrum $\left(400 \mathrm{MHz}, \mathrm{CDCl}_{3}, 298 \mathrm{~K}\right.$ ) of $\mathrm{N}$-(bicyclo[2.2.1] heptan-2-ylmethyl)cyclohexanamine (4)...........22

Figure S24. ${ }^{13} \mathrm{C}$ NMR spectrum $\left(100 \mathrm{MHz}, \mathrm{CDCl}_{3}, 298 \mathrm{~K}\right)$ of $\mathrm{N}$-(bicyclo[2.2.1]heptan-2-ylmethyl)cyclohexanamine (4) ...........22 


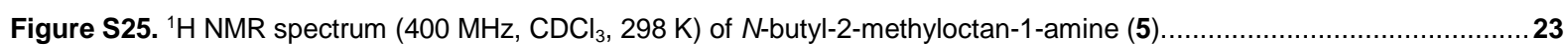

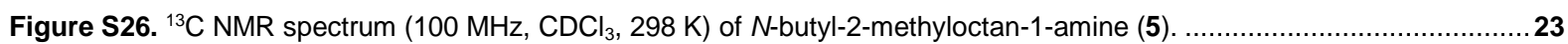

Figure S27. ${ }^{1} \mathrm{H}$ NMR spectrum $\left(400 \mathrm{MHz}, \mathrm{CDCl}_{3}, 298 \mathrm{~K}\right)$ of $\mathrm{N}$-bicyclo[2.2.1]heptan-2-ylmethyl)butan-1-amine (6).................24

Figure S28. ${ }^{13} \mathrm{C}$ NMR spectrum $\left(100 \mathrm{MHz}, \mathrm{CDCl}_{3}, 298 \mathrm{~K}\right)$ of $\mathrm{N}$-bicyclo[2.2.1] heptan-2-ylmethyl)butan-1-amine (6)................24

Figure S29. ${ }^{1} \mathrm{H}$ NMR spectrum $\left(400 \mathrm{MHz}, \mathrm{CDCl}_{3}, 298 \mathrm{~K}\right)$ of $\mathrm{N}$-(2-methyloct-7-en-1-yl)cyclohexanamine (7) ........................25

Figure S30. ${ }^{13} \mathrm{C}$ NMR spectrum $\left(100 \mathrm{MHz}, \mathrm{CDCl}_{3}, 298 \mathrm{~K}\right)$ of $\mathrm{N}$-(2-methyloct-7-en-1-yl)cyclohexanamine (7) .......................25

Figure S31. ${ }^{1} \mathrm{H}$ NMR spectrum $\left(400 \mathrm{MHz}, \mathrm{CDCl}_{3}, 298 \mathrm{~K}\right)$ of $\mathrm{N}$-bicyclo[2.2.1] hept-5-en-2-ylmethyl)cyclohexanamine (8).........26

Figure S32. ${ }^{13} \mathrm{C}$ NMR spectrum $\left(100 \mathrm{MHz}, \mathrm{CDCl}_{3}, 298 \mathrm{~K}\right.$ ) of $\mathrm{N}$-bicyclo[2.2.1] hept-5-en-2-ylmethyl)cyclohexanamine (8)........26

Figure S33. ${ }^{1} \mathrm{H}$ NMR spectrum $\left(400 \mathrm{MHz}, \mathrm{CDCl}_{3}, 298 \mathrm{~K}\right)$ of $N^{1}, N^{8}$-dicyclohexyl-2,7-dimethyloctane-1,8-diamine (9)..............27

Figure S34. ${ }^{13} \mathrm{C}$ NMR spectrum $\left(100 \mathrm{MHz}, \mathrm{CDCl}_{3}, 298 \mathrm{~K}\right)$ of $N^{1}, N^{8}$-dicyclohexyl-2,7-dimethyloctane-1,8-diamine (9).............27

Figure S35. ${ }^{1} \mathrm{H}$ NMR spectrum $\left(400 \mathrm{MHz}, \mathrm{CDCl}_{3}, 298 \mathrm{~K}\right)$ of $\mathrm{N}$-(2-phenylpropyl)cyclohexanamine (10).................................28

Figure S36. ${ }^{13} \mathrm{C}$ NMR spectrum $\left(100 \mathrm{MHz}, \mathrm{CDCl}_{3}, 298 \mathrm{~K}\right)$ of $\mathrm{N}$-(2-phenylpropyl)cyclohexanamine (10).............................28

Figure S37. ${ }^{1} \mathrm{H}$ NMR spectrum $\left(400 \mathrm{MHz}, \mathrm{CDCl}_{3}, 298 \mathrm{~K}\right)$ of $\mathrm{N}$-(2-(4-chlorophenyl)propyl)cyclohexanamine (11)...................29

Figure S38. ${ }^{13} \mathrm{C}$ NMR spectrum $\left(100 \mathrm{MHz}, \mathrm{CDCl}_{3}, 298 \mathrm{~K}\right)$ of $\mathrm{N}$-(2-(4-chlorophenyl)propyl)cyclohexanamine (11) ..................29

Figure S39. ${ }^{1} \mathrm{H}$ NMR spectrum $\left(400 \mathrm{MHz}, \mathrm{CDCl}_{3}, 298 \mathrm{~K}\right)$ of $\mathrm{N}$-(3-(trimethylsilyl)propyl)cyclohexanamine (12)........................30

Figure S40. ${ }^{13} \mathrm{C}$ NMR spectrum $\left(100 \mathrm{MHz}, \mathrm{CDCl}_{3}, 298 \mathrm{~K}\right)$ of $\mathrm{N}$-(3-(trimethylsilyl)propyl)cyclohexanamine (12)......................30

Figure S41. ${ }^{1} \mathrm{H}$ NMR spectrum $\left(400 \mathrm{MHz}, \mathrm{CDCl}_{3}, 298 \mathrm{~K}\right)$ of $\mathrm{N}$-(3-(trimethylsilyl)propyl)butan-1-amine (13) ..........................31

Figure S42. ${ }^{13} \mathrm{C}$ NMR spectrum $\left(100 \mathrm{MHz}, \mathrm{CDCl}_{3}, 298 \mathrm{~K}\right)$ of $\mathrm{N}$-(3-(trimethylsilyl)propyl)butan-1-amine (13).........................31

Figure S43. ${ }^{1} \mathrm{H}$ NMR spectrum $\left(300 \mathrm{MHz}, \mathrm{CDCl}_{3}, 298 \mathrm{~K}\right)$ of $\mathrm{N}$-(2-methyl-3-(trimethylsilyl)propyl)cyclohexanamine (14)..........32

Figure S44. ${ }^{13} \mathrm{C}$ NMR spectrum $\left(75 \mathrm{MHz}, \mathrm{CDCl}_{3}, 298 \mathrm{~K}\right)$ of $\mathrm{N}$-(2-methyl-3-(trimethylsilyl)propyl)cyclohexanamine (14)...........32

Figure S45. ${ }^{1} \mathrm{H}$ NMR spectrum $\left(300 \mathrm{MHz}, \mathrm{CDCl}_{3}, 298 \mathrm{~K}\right)$ of $\mathrm{N}-(2-(($ trimethylsilyl)methyl)hexyl)cyclohexanamine (15).............33

Figure S46. ${ }^{13} \mathrm{C}$ NMR spectrum $\left(75 \mathrm{MHz}, \mathrm{CDCl}_{3}, 298 \mathrm{~K}\right)$ of $\mathrm{N}$-(2-((trimethylsilyl)methyl)hexyl)cyclohexanamine (15)..............33

Figure S47. ${ }^{1} \mathrm{H}$ NMR spectrum $\left(300 \mathrm{MHz}, \mathrm{CDCl}_{3}, 298 \mathrm{~K}\right)$ of 4-methoxy- $\mathrm{N}$-(3-methylnonan-2-yl)aniline (16)..........................34

Figure S48. ${ }^{13} \mathrm{C}$ NMR spectrum $\left(75 \mathrm{MHz}, \mathrm{CDCl}_{3}, 298 \mathrm{~K}\right)$ of 4-methoxy- $\mathrm{N}$-(3-methylnonan-2-yl)aniline (16).........................34

Figure S49. ${ }^{1} \mathrm{H}$ NMR spectrum $\left(300 \mathrm{MHz}, \mathrm{CDCl}_{3}, 298 \mathrm{~K}\right)$ of Desipramine- $\mathrm{N}$-(2-methyl)octylamine (19) ...................................

Figure S50. ${ }^{13} \mathrm{C}$ NMR spectrum $\left(75 \mathrm{MHz}, \mathrm{CDCl}_{3}, 298 \mathrm{~K}\right)$ of Desipramine- $\mathrm{N}$-(2-methyl)octylamine (19). ...............................35

Figure S51. ${ }^{1} \mathrm{H}$ NMR spectrum $\left(300 \mathrm{MHz}, \mathrm{CDCl}_{3}, 298 \mathrm{~K}\right)$ of Desipramine- $\mathrm{N}$-(3-(trimethylsilyl)propylamine (20). (7:3

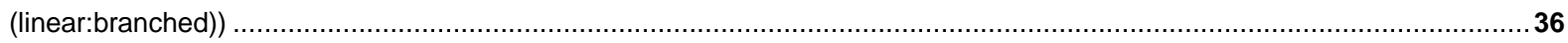

Figure S52. ${ }^{13} \mathrm{C}$ NMR spectrum $\left(75 \mathrm{MHz}, \mathrm{CDCl}_{3}, 298 \mathrm{~K}\right)$ of Desipramine- $\mathrm{N}$-(3-(trimethylsilyl)propylamine (20). (7:3

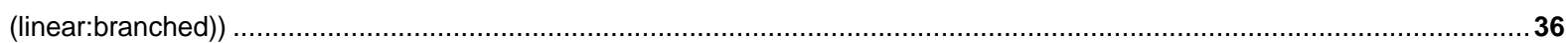

Figure S53. ${ }^{1} \mathrm{H}$ NMR spectrum $\left(300 \mathrm{MHz}, \mathrm{CDCl}_{3}, 298 \mathrm{~K}\right)$ of Fluoxetine- $\mathrm{N}$-(2-methyl)octylamine (22) .................................37

Figure S54. ${ }^{13} \mathrm{C}$ NMR spectrum $\left(75 \mathrm{MHz}, \mathrm{CDCl}_{3}, 298 \mathrm{~K}\right)$ of Fluoxetine- $\mathrm{N}$-(2-methyl)octylamine (22) ................................37

Figure S55. ${ }^{19} \mathrm{~F}$ NMR spectrum $\left(282 \mathrm{MHz}, \mathrm{CDCl}_{3}, 298 \mathrm{~K}\right)$ of Fluoxetine- $\mathrm{N}$-(2-methyl)octylamine (22)................................38

Figure S56. ${ }^{1} \mathrm{H}$ NMR spectrum $\left(300 \mathrm{MHz}, \mathrm{CDCl}_{3}, 298 \mathrm{~K}\right.$ ) of Fluoxetine- $\mathrm{N}$-(3-(trimethylsilyl)propylamine (23), contaminated with $\mathbf{L 2 H}$.

(7:3 (linear:branched))

38

Figure S57. ${ }^{13} \mathrm{C}$ NMR spectrum $\left(100 \mathrm{MHz}, \mathrm{CDCl}_{3}, 298 \mathrm{~K}\right.$ ) of Fluoxetine- $\mathrm{N}$-(3-(trimethylsilyl)propylamine (23), contaminated with

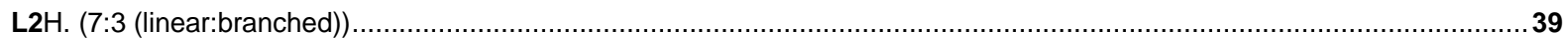

Figure S58. ${ }^{19} \mathrm{~F}$ NMR spectrum $\left(282 \mathrm{MHz}, \mathrm{CDCl}_{3}, 298 \mathrm{~K}\right)$ of Fluoxetine- $\mathrm{N}$-(3-(trimethylsilyl)propylamine (23).....................39 Figure S59. Crude mixture of hydroaminoalkylation of Fluoxetine $(0.25 \mathrm{mmol})$, vinylsilane $(0.37 \mathrm{mmol})$ with $5 \mathrm{~mol} \%$ of L2Ta $\left(\mathrm{CH}_{2} \mathrm{SiMe}_{3}\right)_{3} \mathrm{Cl}$ in $0.5 \mathrm{~mL}$ of $d_{8}$-toluene at $110{ }^{\circ} \mathrm{C}$ for $3 \mathrm{~h}$. HPLC-MS, 10 to $90 \%$ acetonitrile [0.1\% formic acid (FA)] in water $(0.1 \% \mathrm{FA})$ over $10 \mathrm{~min}$ followed by $90 \%$ acetonitrile $(0.1 \% \mathrm{FA})$ in water $(0.1 \% \mathrm{FA})$ over $5 \mathrm{~min}$, flow rate of $1 \mathrm{~mL} / \mathrm{min}$ on an Eclipse XDB-C18, $5 \mu \mathrm{m}, 4.6 \times 150 \mathrm{~mm}, \lambda=263 \mathrm{~nm}$, (Fluoxetine, $t_{R}=7.42 \mathrm{~min}$, area $\left.=54 \%,[\mathrm{M}+\mathrm{H}]^{+}=310\right),\left(23, t_{R}=10.11 \mathrm{~min}\right.$, area $=$ $\left.46 \%,[\mathrm{M}+\mathrm{H}]^{+}=410\right)$ 40

Figure S60. ${ }^{1} \mathrm{H}$ NMR spectrum $\left(300 \mathrm{MHz}, \mathrm{CDCl}_{3}, 298 \mathrm{~K}\right)$ of deuterium labeling experiment. ...........................................41

Figure S61. ${ }^{2} \mathrm{H}$ NMR spectrum $\left(400 \mathrm{MHz}, \mathrm{CHCl}_{3}, 298 \mathrm{~K}\right)$ of deuterium labeling experiment..........................................41

Figure S62. ${ }^{1} \mathrm{H}$ NMR ( $d_{8}$-toluene, $400 \mathrm{MHz}, 298 \mathrm{~K}$ ), $\mathrm{L} 2 \mathrm{Ta}\left(\mathrm{CH}_{2} \mathrm{SiMe}_{3}\right)_{3} \mathrm{Cl}(5 \mathrm{~mol} \%)$ in the presence of 1 equivalent of $\mathrm{N}$ methylcyclohexylamine and 1.5 equivalents of 1 -octene heated at $105{ }^{\circ} \mathrm{C}$ for $5 \mathrm{~min}$. 
Figure S63. ${ }^{2} \mathrm{H}$ NMR (toluene, $400 \mathrm{MHz}, 298 \mathrm{~K}$ ), $\mathrm{L} 2 \mathrm{Ta}\left(\mathrm{CH}_{2} \mathrm{SiMe}_{3}\right)_{3} \mathrm{Cl}$ with 20 equivalents of $\mathrm{PhNHCD}_{3}$ at $105{ }^{\circ} \mathrm{C}$ for $1 \mathrm{~h} . \ldots \ldots .42$

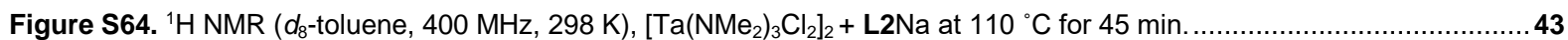

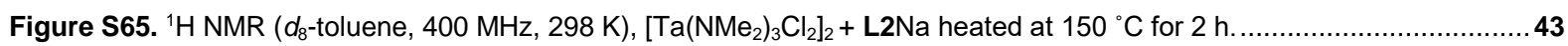

S5. Crystallographic details.

Figure S66. ORTEP representation of the molecular structure of 1. The hydrogen atoms are omitted for clarity. Ellipsoids are drawn

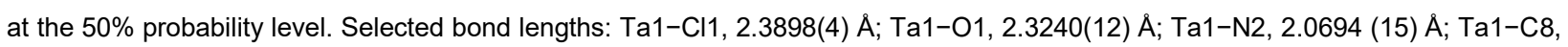
2.1821(16) A; Ta1-C12, 2.1501(16) A; Ta1-C16, 2.1553(18) A. Selected bond angles: O1-Ta1-N2, 60.15(5) ${ }^{\circ}$ O O1-Ta1-Cl1, 137.76(3) ${ }^{\circ}$; N2-Ta1-Cl1, 161.93(4) ${ }^{\circ}$.

Figure S67. ORTEP representation of the molecular structure of 2. The hydrogen atoms are omitted for clarity. Ellipsoids are drawn

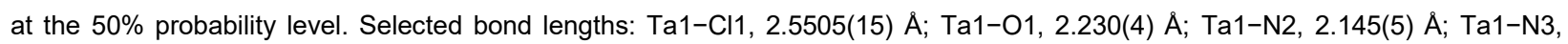
1.991(5) Å; Ta1-N4, 1.977(4) Å; Ta1-N5, 1.985(5) A. Selected bond angles: O1-Ta1-N2, 60.96(16) ; O1-Ta1-Cl1, 82.68(13); N2-Ta1-Cl1, 81.48(18) ${ }^{\circ}$.

45

Figure S68. ORTEP representation of the molecular structure of $\mathbf{2 4}$. The hydrogen atoms are omitted for clarity. Ellipsoids are drawn at the $50 \%$ probability level. Selected bond lengths: Ta1-Cl1, 2.5165(13) $\AA$; Ta1-O1, 2.244(4) $\AA$; Ta1-N2, 2.160(4) $\AA$; Ta1-N3, 1.994(5) Å; Ta1-N4, 1.990(5) Å; Ta1-N5, 1.977(4) Å. Selected bond angles: O1-Ta1-N2, 61.26(15); O1-Ta1-Cl1, $84.05(11)^{\circ}$; N2-Ta1-Cl1, 82.00(12) 


\section{S1. Materials}

All reactions were performed under an $\mathrm{N}_{2}$ atmosphere using Schlenk or glovebox techniques, unless otherwise stated. All amines (besides $\mathrm{PhNHCD}_{3}, \mathrm{PhNDCH}_{3}$ and $\left.\mathrm{PhNDCD}_{3}\right)^{[1]}$ and alkenes were commercially available, dried over $\mathrm{CaH}{ }_{2}$ and distilled and degassed or sublimated prior to use in catalytic experiments. Hexenyltrimethylsilane was prepared according to

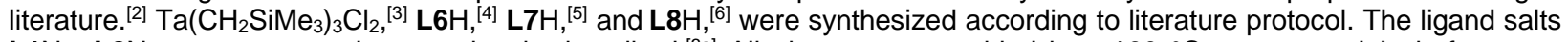
L1Na-L8Na were prepared as previously described. ${ }^{[3 a]}$ All glassware was dried in a $160{ }^{\circ} \mathrm{C}$ oven overnight before use. Toluene and hexanes were dried over activated alumina columns and stored over activated molecular sieves $(4 \AA)$. $d_{8}$ Toluene was dried over sodium/ketyl and distilled prior to use. Experiments conducted on an NMR tube scale were performed in J-Young NMR tubes $\left(8\right.$ " x $5 \mathrm{~mm}$ ) sealed with screw-type Teflon caps. In a glovebox under $\mathrm{N}_{2}$ atmosphere the desired amount of amine, alkene, catalyst, additives and internal standards were dissolved or suspended in $0.5 \mathrm{~mL}$ of $d_{8}$ toluene and transferred to $\mathrm{J}$-Young tubes for end point reactions or $5 \mathrm{~mL}$ round bottom flasks for kinetic studies. The reaction vessel was placed in an oil bath at $105{ }^{\circ} \mathrm{C}$. For the kinetic studies the samples were taken by an argon purged syringe and quenched in $0.5 \mathrm{~mL}$ of $d_{8}$-toluene. The standard condition for the hydroaminoalkylation reaction is $5 \mathrm{~mol} \%$ of the catalyst, $0.5 \mathrm{mmol}$ of the amine substrate and $0.75 \mathrm{mmol}$ of the alkene substrate heated at $105^{\circ} \mathrm{C}$.

\section{S2. Instrumentation}

${ }^{1} \mathrm{H},{ }^{2} \mathrm{H},{ }^{19} \mathrm{~F}$ and ${ }^{13} \mathrm{C}$ NMR spectra were recorded on Bruker 300 and $400 \mathrm{MHz}$ Avance spectrometers at ambient temperature. Chemical shifts $(\delta)$ are given relative to the corresponding residual proton solvent and are reported in parts per million $(\mathrm{ppm})$. Coupling constants $(\mathrm{J})$ are given in Hertz $(\mathrm{Hz})$. The following abbreviations are used to indicate signal multiplicity: $\mathrm{S}=$ singlet, $d=$ doublet, $t=$ triplet, $q=$ quartet, $m=$ multiplet, and $b r=$ broad. Assignment of the signals was carried out using 1D $\left({ }^{1} \mathrm{H},{ }^{2} \mathrm{H},{ }^{13} \mathrm{C}\right.$ and $\left.{ }^{19} \mathrm{~F}\right)$ NMR experiments or HMBC and HSQC when specified. Mass spectra (MS) were measured at the Department of Chemistry on a Kratos MS-50 spectrometer using a Bruker maXis Ultra-High Resolution tandem TOF (UHRQq-TOF) mass spectrometer using a positive electrospray ionization source. Fragment signals are given in mass per charge number $(\mathrm{m} / \mathrm{z})$. Elemental analyses were recorded on a Carlo Erba EA 1108 elemental analyzer. Single crystal X-ray diffraction data were collected using a Bruker X8 APEX or Bruker APEX DUO diffractometer.

\section{S3. Synthesis and characterization of ligands and complexes:}

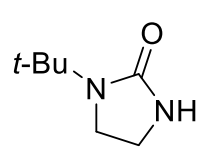

[1-(tert-butyl)imidazolidin-2-one], L2H. Under nitrogen, a solution of 2-chloroethyl isocyanate $(6.80 \mathrm{~g}, 64$ $\mathrm{mmol})$ in THF $(50 \mathrm{~mL})$ was added dropwise to a stirring solution of tert-butylamine $(4.28 \mathrm{~g}, 58.5 \mathrm{mmol})$ in THF $(20 \mathrm{~mL})$ at $-20{ }^{\circ} \mathrm{C}$. The solution was brought to room temperature overnight. The resulting reaction mixture was treated with $\mathrm{NaH}(6.8 \mathrm{~g}, 283 \mathrm{mmol})$ and heated at $65^{\circ} \mathrm{C}$ overnight under an inert atmosphere. The mixture was brought to dryness and treated with saturated $\mathrm{NH}_{4} \mathrm{Cl}(100 \mathrm{~mL})$ and EtOAc $(200 \mathrm{~mL})$. The organic layer was separated, and the aqueous layer was extracted with EtOAc $(3 \times 50 \mathrm{~mL})$. The combined organic fractions were dried over $\mathrm{Na}_{2} \mathrm{SO}_{4}$ and brought to dryness under vacuum forming a yellow oil. Hexanes $(50 \mathrm{~mL})$ were then added resulting in the formation of a solid at the bottom of the round bottom flask. The mother liquor was removed by filtration. This process was repeated 3 times. Storing the combined hexane solutions at low temperature resulted in the formation of colorless crystals that were later filtered and dried in vacuo to afford the pure product. Yield $(1.12 \mathrm{~g}, 13 \%) .{ }^{1} \mathrm{H} \mathrm{NMR}\left(\mathrm{CDCl}{ }_{3}\right.$, $300 \mathrm{MHz}, 298 \mathrm{~K}): \delta 4.37$ (br s, $1 \mathrm{H}, \mathrm{NH}), 3.49-3.40\left(\mathrm{~m}, 2 \mathrm{H},{ }^{\mathrm{tBuNCH}} \mathrm{N}_{2}\right)$, 3.33-3.23 (m, 2H, HNCH$), 1.36\left(\mathrm{~s}, 9 \mathrm{H}, \mathrm{C}\left(\mathrm{CH}_{3}\right)_{3}\right) \mathrm{ppm}$. ${ }^{13} \mathrm{C} \mathrm{NMR}\left(\mathrm{CDCl}_{3}, 75 \mathrm{MHz}, 298 \mathrm{~K}\right): \delta 163.15(\mathrm{C}=\mathrm{O}), 52.96\left(\mathrm{C}\left(\mathrm{CH}_{3}\right)_{3}\right), 43.73\left({ }^{\mathrm{tBu}} \mathrm{NCH}_{2}\right), 38.13(\mathrm{HNCH}), 27.67\left(\mathrm{C}\left(\mathrm{CH}_{3}\right)_{3}\right) \mathrm{ppm}$. HRMS (ESI): $m / z$ calcd for $\mathrm{C}_{7} \mathrm{H}_{14} \mathrm{~N}_{2} \mathrm{O}\left[\mathrm{M}+\mathrm{Na}^{+}\right]$: 165.10039. Found: 165.1001. Anal. Calcd. for $\mathrm{C}_{7} \mathrm{H}_{14} \mathrm{~N}_{2} \mathrm{O}: \mathrm{C}, 59.12 ; \mathrm{H}, 9.92$; N, 19.70; Found: C, 59.12; H, 10.29; N, 19.71.

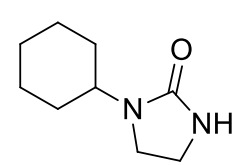

[1-cyclohexylimidazolidin-2-one], L3H. Prepared following the general procedure outlined above: 2chloroethylisocyanate $(1.11 \mathrm{~g}, 10.5 \mathrm{mmol})$, cyclohexylamine $(0.99 \mathrm{~g}, 10 \mathrm{mmol}), \mathrm{NaH}(0.24 \mathrm{~g}, 10 \mathrm{mmol})$. The pure product was obtained by filtration. Yield $(0.44 \mathrm{~g}, 27 \%) .{ }^{1} \mathrm{H} \mathrm{NMR}\left(\mathrm{CDCl}_{3}, 300 \mathrm{MHz}, 298 \mathrm{~K}\right): \delta$ 5.41 (br s, $1 \mathrm{H}, \mathrm{NH}), 3.77-3.58(\mathrm{~m}, 1 \mathrm{H}, \mathrm{NCH}), 3.43\left(\mathrm{~s}, 4 \mathrm{H}, \mathrm{CH}_{2} \mathrm{CH}_{2} \mathrm{NH}\right), 1.92-1.52\left(\mathrm{~m}, 5 \mathrm{H}, \mathrm{CH}_{2}\right)$ 1.46-1.21 $\left(\mathrm{m}, 2 \mathrm{H}, \mathrm{CH}_{2}\right), 1.18-0.95(\mathrm{~m}, 1 \mathrm{H}, \mathrm{NC}(H) \mathrm{H}) \mathrm{ppm} .{ }^{13} \mathrm{C} \mathrm{NMR}\left(\mathrm{CDCl}_{3}, 75 \mathrm{MHz}, 298 \mathrm{~K}\right): \delta 162.52(C=\mathrm{O})$, $51.15\left({ }^{\mathrm{tBu} N} \mathrm{NH}_{2}\right), 40.71,38.76,30.39,25.64 \mathrm{ppm}$. HRMS (ESI): $\mathrm{m} / \mathrm{z}$ calcd for $\mathrm{C}_{9} \mathrm{H}_{16} \mathrm{~N}_{2} \mathrm{ONa}\left[\mathrm{M}+\mathrm{Na}^{+}\right]$: 191.1160. Found: 191.1159.

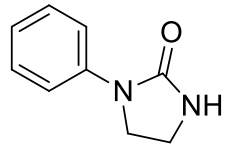

[1-phenylimidazolidin-2-one], L4H. Prepared following the general procedure outlined above: 2chloroethylisocyanate $(1.05 \mathrm{~g}, 10 \mathrm{mmol})$, aniline $(0.93 \mathrm{~g}, 10 \mathrm{mmol}), \mathrm{NaH}(0.24 \mathrm{~g}, 10 \mathrm{mmol})$. Yield $(0.42 \mathrm{~g}$, 26\%). ${ }^{1} \mathrm{H}$ NMR $\left(\mathrm{CDCl}_{3}, 300 \mathrm{MHz}, 298 \mathrm{~K}\right): \delta 7.53\left(\mathrm{~d},{ }^{3} \mathrm{~J}_{\mathrm{H}-\mathrm{H}}=8.2 \mathrm{~Hz}, 2 \mathrm{H}, \mathrm{o}_{-} \mathrm{C}_{6} \mathrm{H}_{5}\right), 7.39-7.28\left(\mathrm{~m}, 2 \mathrm{H}, \mathrm{o}^{-}\right.$ $\left.\mathrm{C}_{6} H_{5}\right), 7.05\left(\mathrm{t},{ }^{3} \mathrm{~J}_{\mathrm{H}-\mathrm{H}}=7.3 \mathrm{~Hz}, 1 \mathrm{H}, p-\mathrm{C}_{6} \mathrm{H}_{5}\right), 3.92\left(\mathrm{t},{ }^{3} \mathrm{~J}_{\mathrm{H}-\mathrm{H}}=7.0 \mathrm{~Hz}, 2 \mathrm{H}, \mathrm{NCH}_{2}\right), 3.57\left(\mathrm{t},{ }^{3} \mathrm{~J}_{\mathrm{H}-\mathrm{H}}=7.1 \mathrm{~Hz}, 2 \mathrm{H}\right.$, $\left.\mathrm{NCH}_{2}\right)$ ppm. ${ }^{13} \mathrm{C} \mathrm{NMR}\left(\mathrm{CDCl}_{3}, 75 \mathrm{MHz}, 298 \mathrm{~K}\right): \delta 160.27(\mathrm{C}=\mathrm{O}), 140.15\left(i-\mathrm{C}_{6} \mathrm{H}_{5}\right), 128.92\left(m-\mathrm{C}_{6} \mathrm{H}_{5}\right)$, $122.83\left(p-\mathrm{C}_{6} \mathrm{H}_{5}\right), 118.09\left(o-\mathrm{C}_{6} \mathrm{H}_{5}\right), 45.49\left(\mathrm{NCH}_{2}\right), 37.70\left(\mathrm{HNCH}_{2}\right)$ ppm. HRMS (ESI): $\mathrm{m} / z$ calcd for $\quad \mathrm{C}_{9} \mathrm{H}_{10} \mathrm{~N}_{2} \mathrm{ONa}\left[\mathrm{M}+\mathrm{Na}^{+}\right]$: 185.0691. Found: 185.0691. 


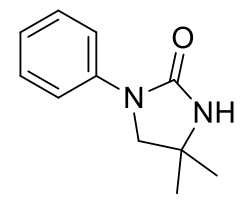

[4,4-dimethyl-1-phenylimidazolidin-2-one], L5H. The title compound was synthesized using 3(benzoyloxy)-1-isobutyl-1-phenylurea $(1.25 \mathrm{~g}, \quad 4.00 \mathrm{mmol}), \quad \operatorname{tris}(2,2$ '-bipyridine $)$ ruthenium(II) hexafluorophosphate $(0.069 \mathrm{~g}, 0.08 \mathrm{mmol})$, triethylamine $(0.61 \mathrm{~mL}, 4.40 \mathrm{mmol})$ in $\mathrm{MeCN}(20 \mathrm{~mL}, 0.2 \mathrm{M})$ in a $20 \mathrm{~mL}$ microwave vial sealed with a Teflon cap and a stir bar. The vial was placed in an ice bath and purged with Argon for $10 \mathrm{~min}$ (open outlet). The vial was positioned $2 \mathrm{~cm}$ from the light source (40W Kessil Tuna Blue LED lamp) and allowed to stir overnight at ambient temperature (using fan cooling). Upon completion, the reaction was concentrated under reduced pressure, diluted in $\mathrm{CH}_{2} \mathrm{Cl}_{2}(50 \mathrm{~mL})$ and extracted three times with sodium bicarbonate $(3 \times 20 \mathrm{~mL})$ to remove the benzoic acid. The organic phase was collected, dried with $\mathrm{Na}_{2} \mathrm{SO}_{4}$, filtered, and concentrated under reduced pressure. The title compound was purified by gradient column chromatography (10-20\% EtOAc in $\left.\mathrm{CH}_{2} \mathrm{Cl}_{2}\right)$. Yield $(0.380 \mathrm{~g}, 50 \%)$. ${ }^{1} \mathrm{H}$ NMR $\left(\mathrm{CDCl}_{3}, 300 \mathrm{MHz}, 298 \mathrm{~K}\right): \delta 7.53\left(\mathrm{~d}, \mathrm{~J}_{\mathrm{H}-\mathrm{H}}=7.9 \mathrm{~Hz}, 2 \mathrm{H}, m-\mathrm{C}_{6} \mathrm{H}_{5}\right)$, 7.39-7.29 (m, 2H, o- $\left.\mathrm{C}_{6} H_{5}\right), 7.04\left(\mathrm{t}, \mathrm{J}_{\mathrm{H}-\mathrm{H}}=7.3 \mathrm{~Hz}, 2 \mathrm{H}, p-\mathrm{C}_{6} \mathrm{H}_{5}\right), 5.27$ (br s, $\left.1 \mathrm{H}, \mathrm{NH}\right), 3.63\left(\mathrm{~s}, 2 \mathrm{H}, \mathrm{CH}_{2}\right), 1.39(\mathrm{~s}, 3 \mathrm{H}, \mathrm{CH}) \mathrm{ppm}$. ${ }^{13} \mathrm{C}$ NMR $\left(\mathrm{CDCl}_{3}, 75 \mathrm{MHz}, 298 \mathrm{~K}\right): \delta 158.33(C=\mathrm{O}), 140.37\left(i-C_{6} \mathrm{H}_{5}\right), 128.91\left(m-C_{6} \mathrm{H}_{5}\right), 122.63\left(p-C_{6} \mathrm{H}_{5}\right), 117.87\left(p-C_{6} \mathrm{H}_{5}\right)$,

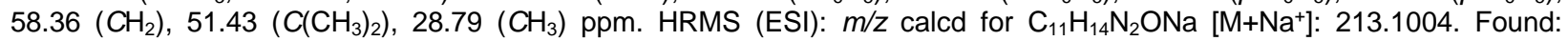
213.1012.

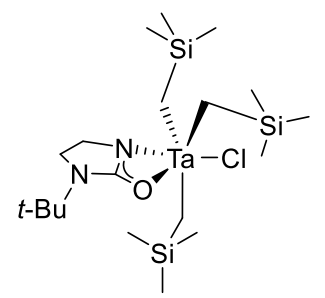

[L2Ta( $\left.\mathrm{CH}_{2} \mathrm{SiMe}_{3}\right)_{3} \mathrm{Cl}$ ], 1. To a stirring solution of $\mathrm{Ta}\left(\mathrm{CH}_{2} \mathrm{SiMe}_{3}\right)_{3} \mathrm{Cl}_{2}(200 \mathrm{mg}, 0.39 \mathrm{mmol})$ in $2 \mathrm{~mL}$ of toluene, a suspension of $\mathrm{L} 2 \mathrm{Na}(71 \mathrm{mg}, 0.43 \mathrm{mmol})$ in $2 \mathrm{~mL}$ of toluene was added dropwise. The resulting yellow suspension was left to stir at room temperature for overnight. The toluene was evaporated, and the resulting yellow oil was dissolved in $4 \mathrm{~mL}$ of hexane and then filtered over celite. The yellow solution was concentrated to half and left in the freezer at $-30{ }^{\circ} \mathrm{C}$ for 1 hour to afford yellow crystals suitable for X-ray diffraction studies. Yield $(150 \mathrm{mg}, 62 \%)$. ${ }^{1} \mathrm{H}$ NMR $\left(d_{8}-\right.$ toluene, $400 \mathrm{MHz}, 298 \mathrm{~K}$ ): $\delta 3.34$ (t, $J_{\mathrm{H}-\mathrm{H}}=8.0 \mathrm{~Hz}, 2 \mathrm{H}, \mathrm{CH}_{2}$ ), 2.77 (t, $\left.\mathrm{J}_{\mathrm{H}-\mathrm{H}}=8.0 \mathrm{~Hz}, 2 \mathrm{H}, \mathrm{CH}_{2}\right), 1.50$ (s, 6H, CH $\left.\mathrm{CH}_{2} \mathrm{Si}\right), 1.09\left(\mathrm{~s}, 9 \mathrm{H}, \mathrm{NC}\left(\mathrm{CH}_{3}\right)_{3}\right), 0.32(\mathrm{~s}, 27 \mathrm{H}, \mathrm{SiCH}) \mathrm{ppm} .{ }^{13} \mathrm{C}$ NMR $\left(d_{8}\right.$-toluene, $101 \mathrm{MHz}$, 298K): $\delta 171.4(\mathrm{C}=\mathrm{O}), 90.0\left(\mathrm{CH}_{2} \mathrm{Si}\right), 53.7\left(\mathrm{NC}\left(\mathrm{CH}_{3}\right)_{3}\right), 45.4\left(\mathrm{CH}_{2}\right), 44.5\left(\mathrm{CH}_{2}\right), 27.9\left(\mathrm{NC}\left(\mathrm{CH}_{3}\right)_{3}\right), 2.7$ $\left(\mathrm{SiCH}_{3}\right)$ ppm. Anal. Calcd. for $\mathrm{C}_{19} \mathrm{H}_{47} \mathrm{CIN}_{2} \mathrm{OSi}{ }_{3} \mathrm{Ta}$ : C, 36.79; H, 7.64; N, 4.52; Found: C, 36.44; H, 7.69; N, 4.59.

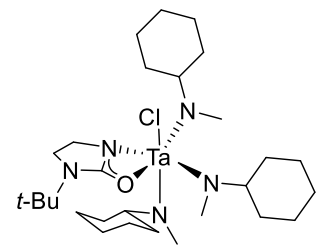

[L2Ta(NMeCy) ${ }_{3} \mathbf{C l}$ ], 2. To a stirring solution of $\mathrm{L2Ta}\left(\mathrm{CH}_{2} \mathrm{SiMe}_{3}\right)_{3} \mathrm{Cl}(50 \mathrm{mg}, 0.08 \mathrm{mmol})$ in $2 \mathrm{ml}$ of toluene, 3 equivalents of $\mathrm{N}$-methylcyclohexylamine $(27 \mathrm{mg}, 0.24 \mathrm{mmol})$ was added. The yellow solution was heated at $110{ }^{\circ} \mathrm{C}$ for 24 hours. The toluene was dried off and the resulting brown oil was dissolved in $2 \mathrm{~mL}$ of hexane. Yellow crystals suitable for X-ray diffraction studies were grown after 1 week at $-30{ }^{\circ} \mathrm{C}$. The yellow crystals were recrystallized three times. Yield (18 mg, 32\%). An analytically pure sample could not be obtained. ${ }^{1} \mathrm{H}$ NMR $\left(d_{8}\right.$-toluene, $\left.400 \mathrm{MHz}, 298 \mathrm{~K}\right): \delta 3.96-$ 3.37 (br m, 9H, NCH $\mathrm{N}_{3}$ ), 3.01 (dd, $\left.J_{\mathrm{H}-\mathrm{H}}=16.6,8.2 \mathrm{~Hz}, 2 \mathrm{H}, \mathrm{NCH}_{2}\right), 2.31$ (dd, $J_{\mathrm{H}-\mathrm{H}}=27.0,5.9 \mathrm{~Hz}, 2 \mathrm{H}$, $\mathrm{NCH}_{2}$ ), $2.03-1.30$ (br m, 26H, $\mathrm{CH}_{2}$ ), 1.24 (s, 9H, NC( $\left.\left.\mathrm{CH}_{3}\right)_{3}\right), 1.21-0.79$ (br m, 7H, CH$)_{2}$ ppm. ${ }^{13} \mathrm{C}$ NMR ( $d_{8}$-toluene, $\left.101 \mathrm{MHz}, 298 \mathrm{~K}\right): \delta 170.7,60.4,58.7,52.8,46.3,44.8,33.9,33.6,32.0,30.3,29.1,28.9,28.2,28.1$, 26.9, 26.8, 26.6, 26.5, 26.4, 25.2 ppm. Anal. Calcd. for $\mathrm{C}_{28} \mathrm{H}_{56} \mathrm{CIN}_{5} \mathrm{OTa}$ : C, 48.45; H, 7.99; N, 10.09; Found: C, 46.36; $\mathrm{H}$, $7.30 ; \mathrm{N}, 9.52$.<smiles>CN[Te](Cl)(Cl)(N(C)C)N1C(=O)N(C(C)(C)C)CC1C(C)C</smiles>

[L6Ta(NMe2) $\left.{ }_{3} \mathrm{Cl}\right]$, 24. Prepared following the procedure outlined for 1: from $\mathrm{Ta}\left(\mathrm{NMe}_{2}\right)_{3} \mathrm{Cl}_{2}(100$ $\mathrm{mg}, 0.026 \mathrm{mmol}$ ) in $4 \mathrm{~mL}$ of toluene and sodium (S)-3-(isopropyl)-5-(tert-butyl)-2-oxoimidazolidin1-ide (53 mg, $0.26 \mathrm{mmol}$ ) in $4 \mathrm{~mL}$ of toluene. The filtered solution over celite was dried and the resulting oil afforded very small amounts of crystals at room temperature. The few crystals obtained could only be used for X-ray diffraction studies. 
Table S1. Ligand scope

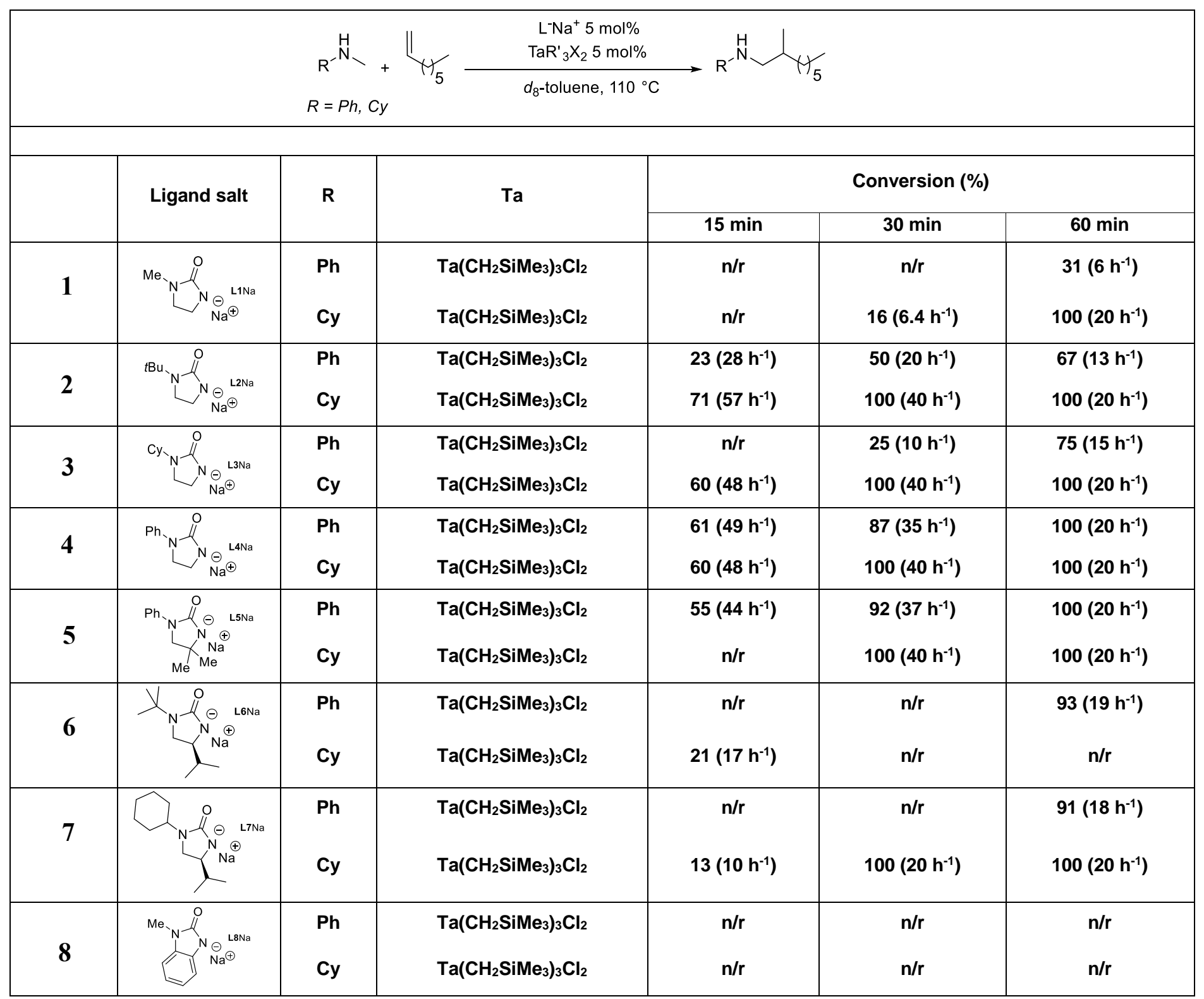

Reaction conditions: amine $(0.5 \mathrm{mmol}), 1$-octene $(0.5 \mathrm{mmol}),[\mathrm{Ta}](0.025 \mathrm{mmol})$, ligand salt $(0.025 \mathrm{mmol}), \mathrm{d}_{8}$-toluene $(0.5 \mathrm{~g})$. Conversion determined by ${ }^{1} \mathrm{H}$ NMR spectroscopy. TOF values are given in brackets where applicable. 
Table S2. Solid- $G$ parameters $G_{L}$ and $G_{\text {space }}$ calculated for ureate tantalum catalysts

\begin{tabular}{|c|c|c|}
\hline (\%) & $G_{\llcorner}$ & $G_{\text {space }}$ \\
\hline 1-(tert-butyl)imidazolidin-2-one ligand (L2H) in 1 & 24.94 & 20.32 \\
\hline 3-(2,6-dimethylphenyl)-piperidylureate ligand in 1a & 28.98 & 18.51 \\
\hline
\end{tabular}
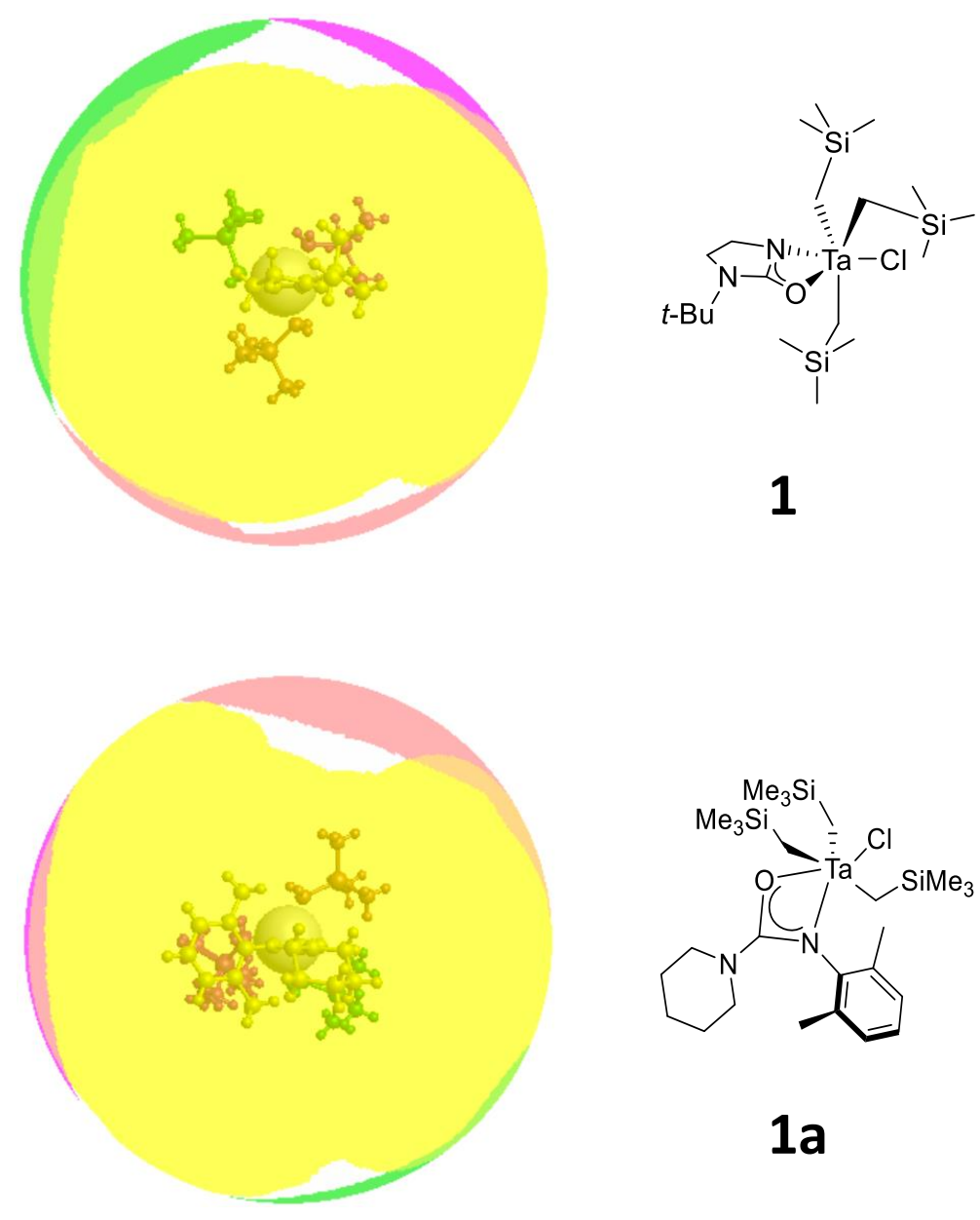

1

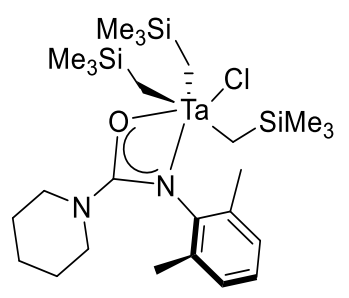

$1 a$

Figure S1. Solid-G output for complex 1 (top) and 1a (bottom). Percentage of occupancy by ureate ligand shown in yellow. 


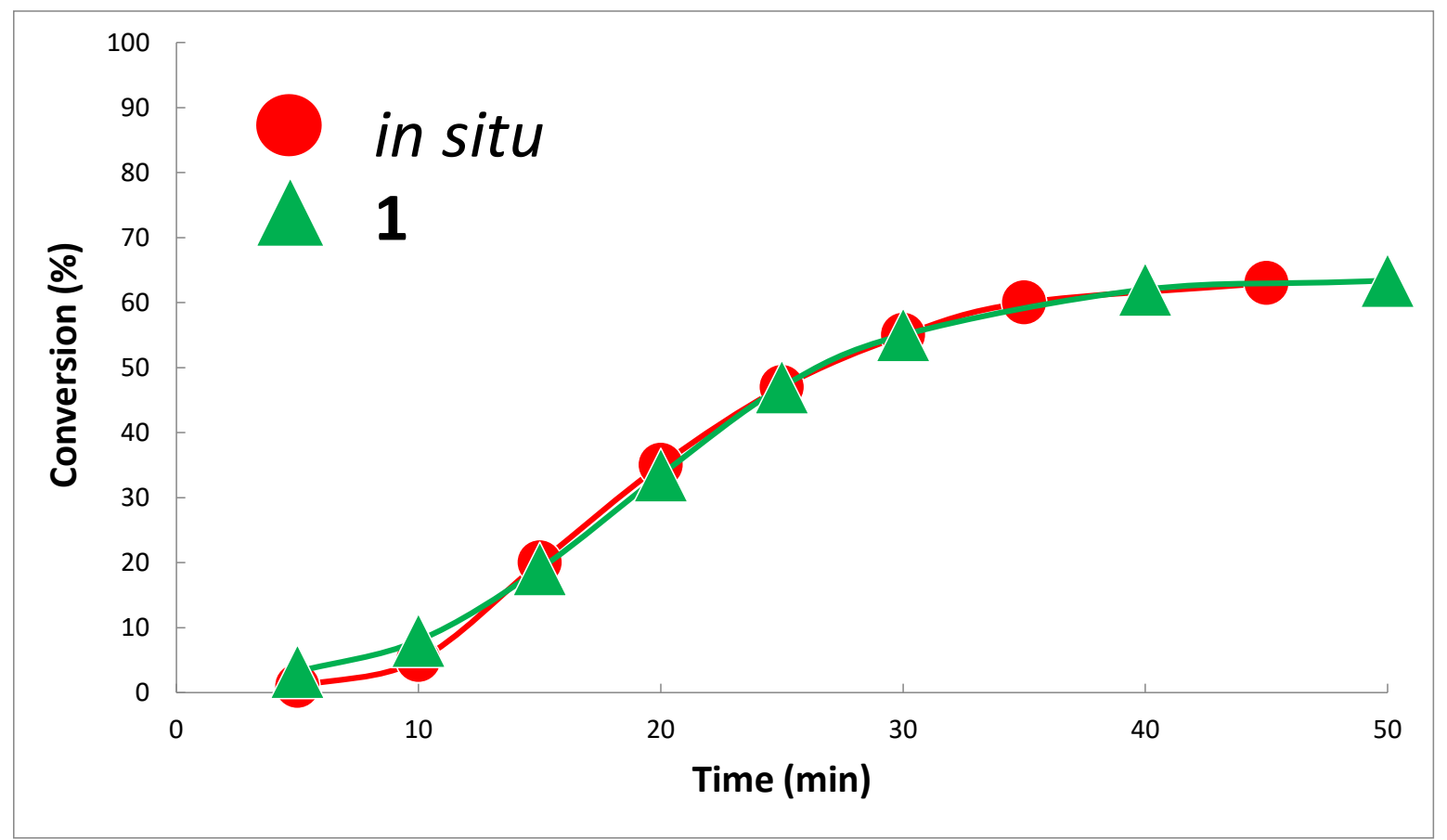

Figure S2. Conversion vs time plot observed for the hydroaminoalkylation reaction of $\mathrm{Ta}\left(\mathrm{CH}_{2} \mathrm{SiMe}_{3}\right)_{3} \mathrm{Cl}{ }_{2}+\mathrm{L}_{2} \mathrm{Na}$ (in situ) or $\mathbf{1}$ with $\mathrm{N}$-methylaniline and 1 -octene at $110^{\circ} \mathrm{C}$ in $d_{8}$-toluene.

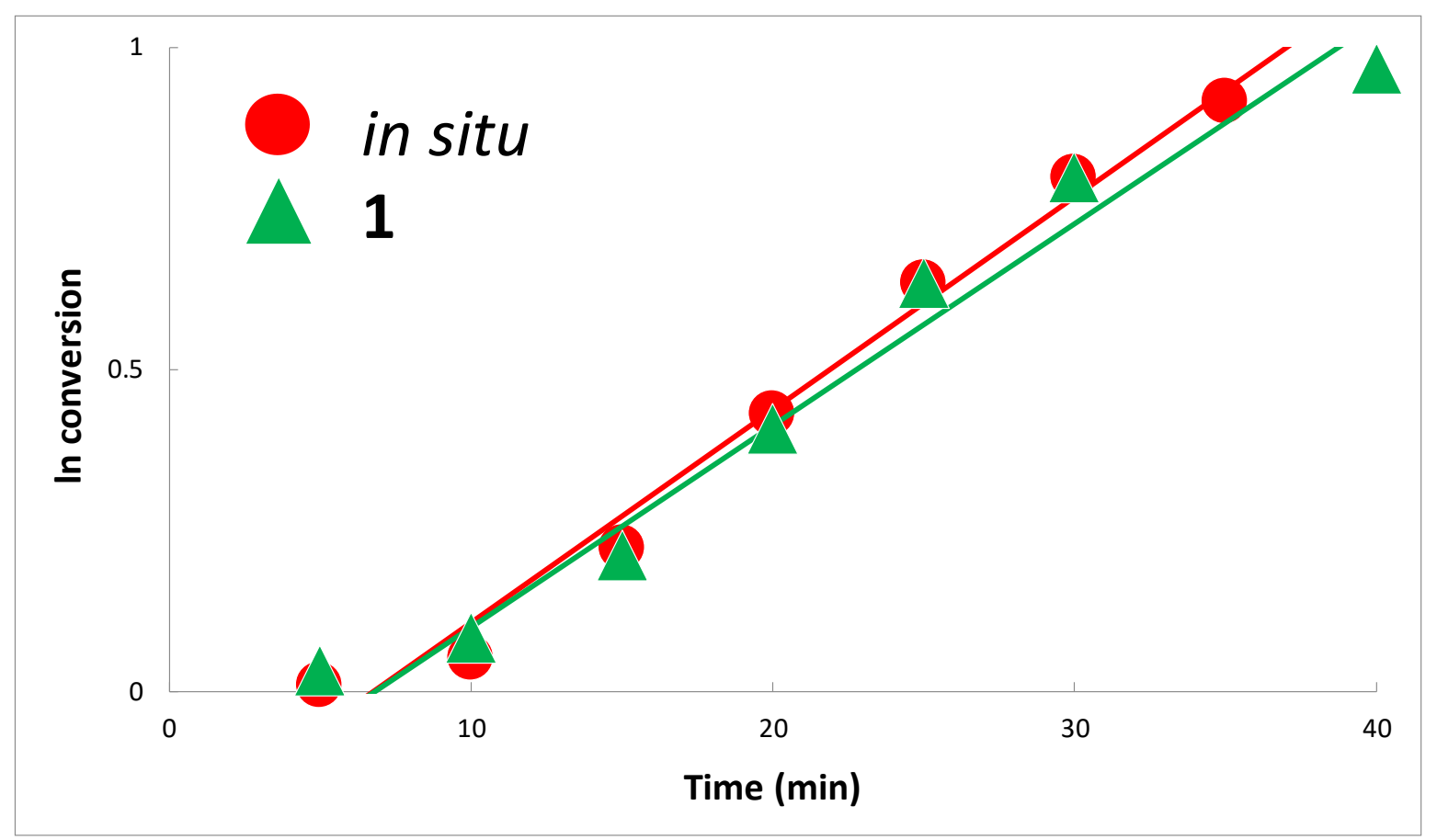

Figure S3. Comparison of $k_{\text {obs }}$ values observed for the hydroaminoalkylation reaction of $\mathrm{Ta}\left(\mathrm{CH}_{2} \mathrm{SiMe}_{3}\right)_{3} \mathrm{Cl}_{2}+\mathrm{L}_{2} \mathrm{Na}_{(\text {in }}$ situ) or 1 with $\mathrm{N}$-methylaniline and 1 -octene at $110{ }^{\circ} \mathrm{C}$ in $d_{8}$-toluene. 


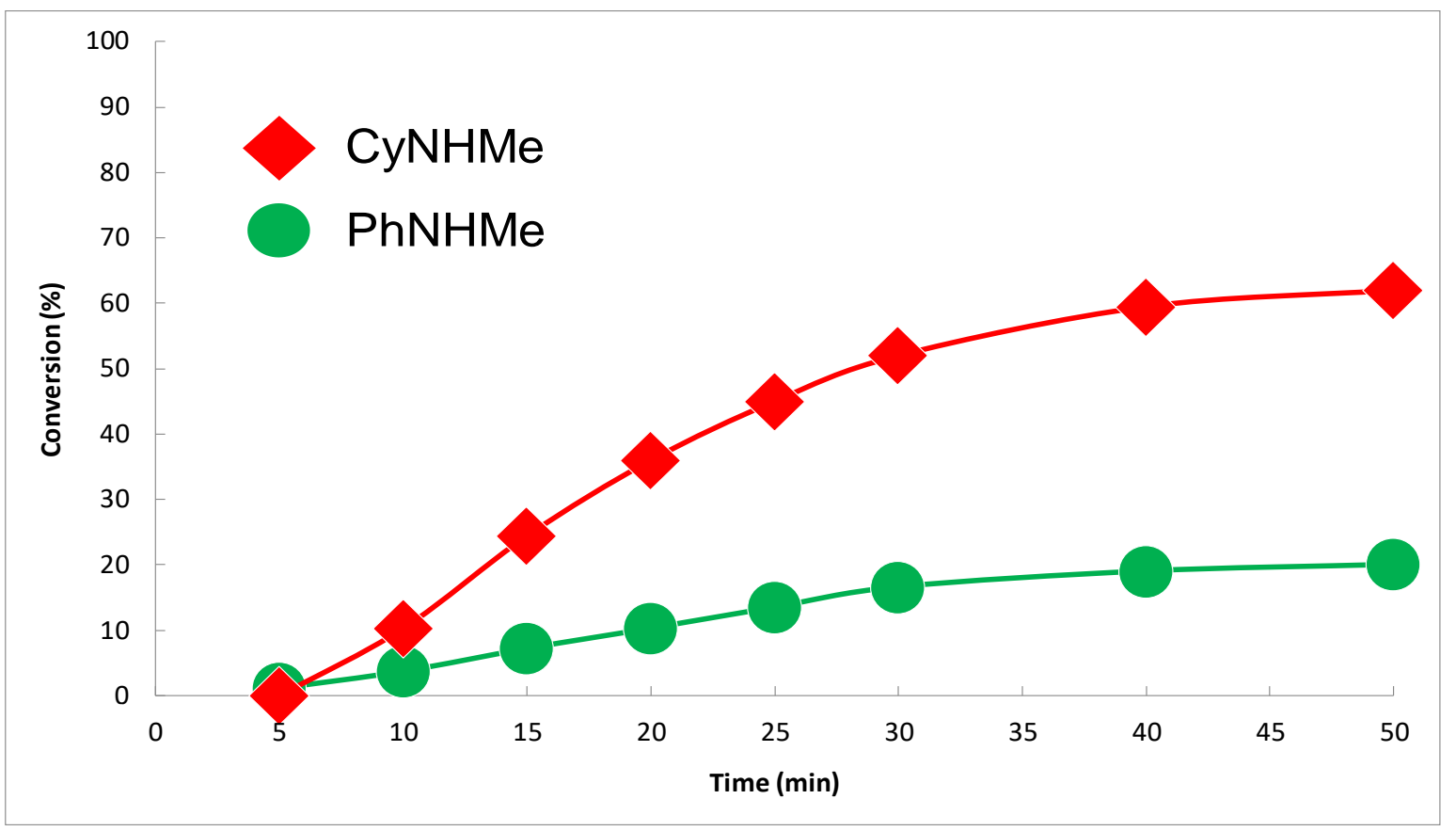

Figure S4. Conversion vs time plot observed for $0.5 \mathrm{mmol} N$-methylcyclohexylamine (diamonds), $0.5 \mathrm{mmol} N$-methylaniline (circles), $0.5 \mathrm{mmol} 1$-octene and $5 \mathrm{~mol} \% 1$ in $0.5 \mathrm{~mL}$ of $d_{8}$-toluene at $105^{\circ} \mathrm{C}$.

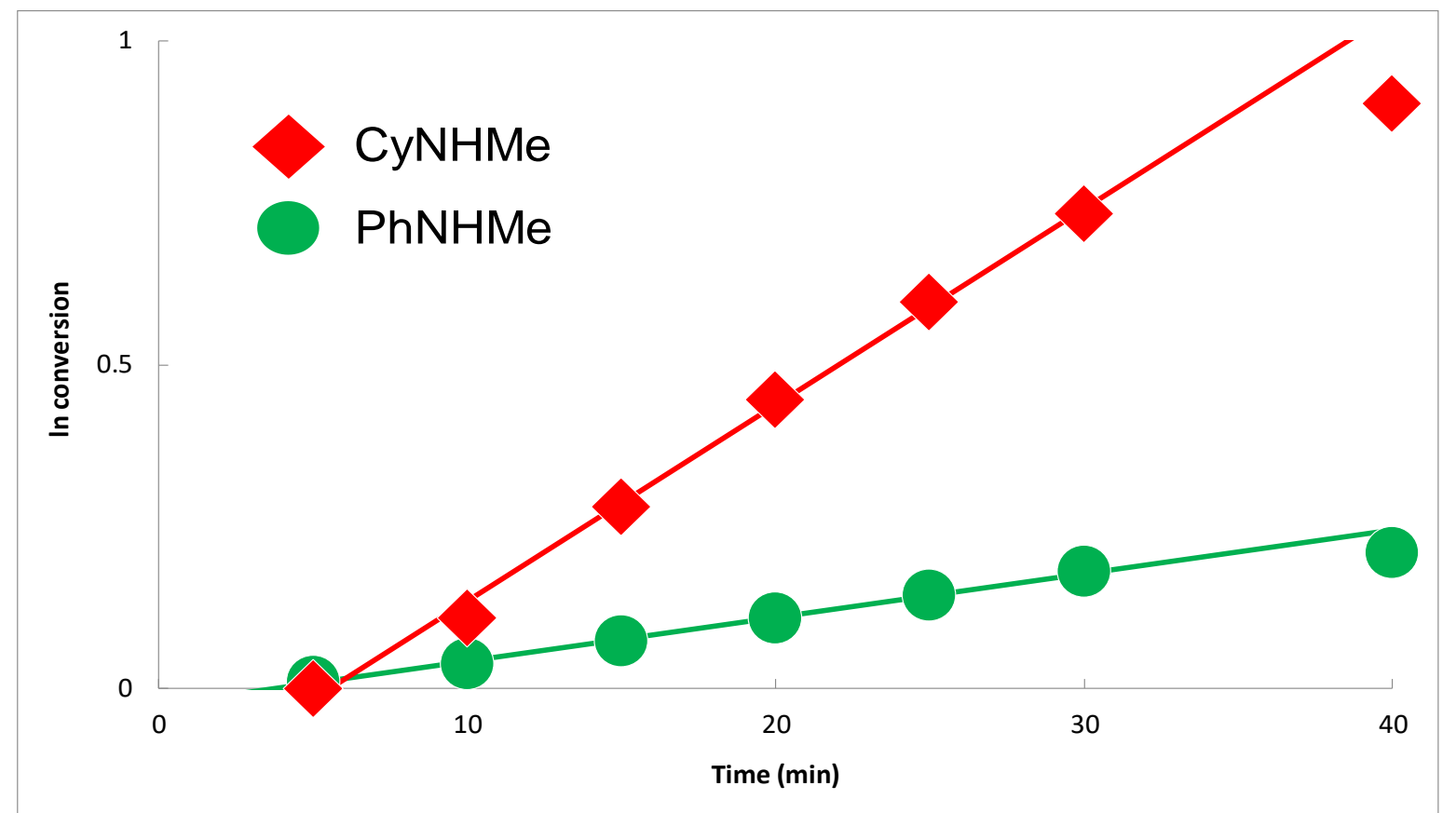

Figure S5. Comparison of $k_{\text {obs }}$ values observed for $0.5 \mathrm{mmol} N$-methylcyclohexylamine (diamonds), $0.5 \mathrm{mmol} N$ methylaniline (circles), $0.5 \mathrm{mmol} 1$-octene and $5 \mathrm{~mol} \% 1$ in $0.5 \mathrm{~mL}$ of $d_{8}$-toluene at $105{ }^{\circ} \mathrm{C}$. 


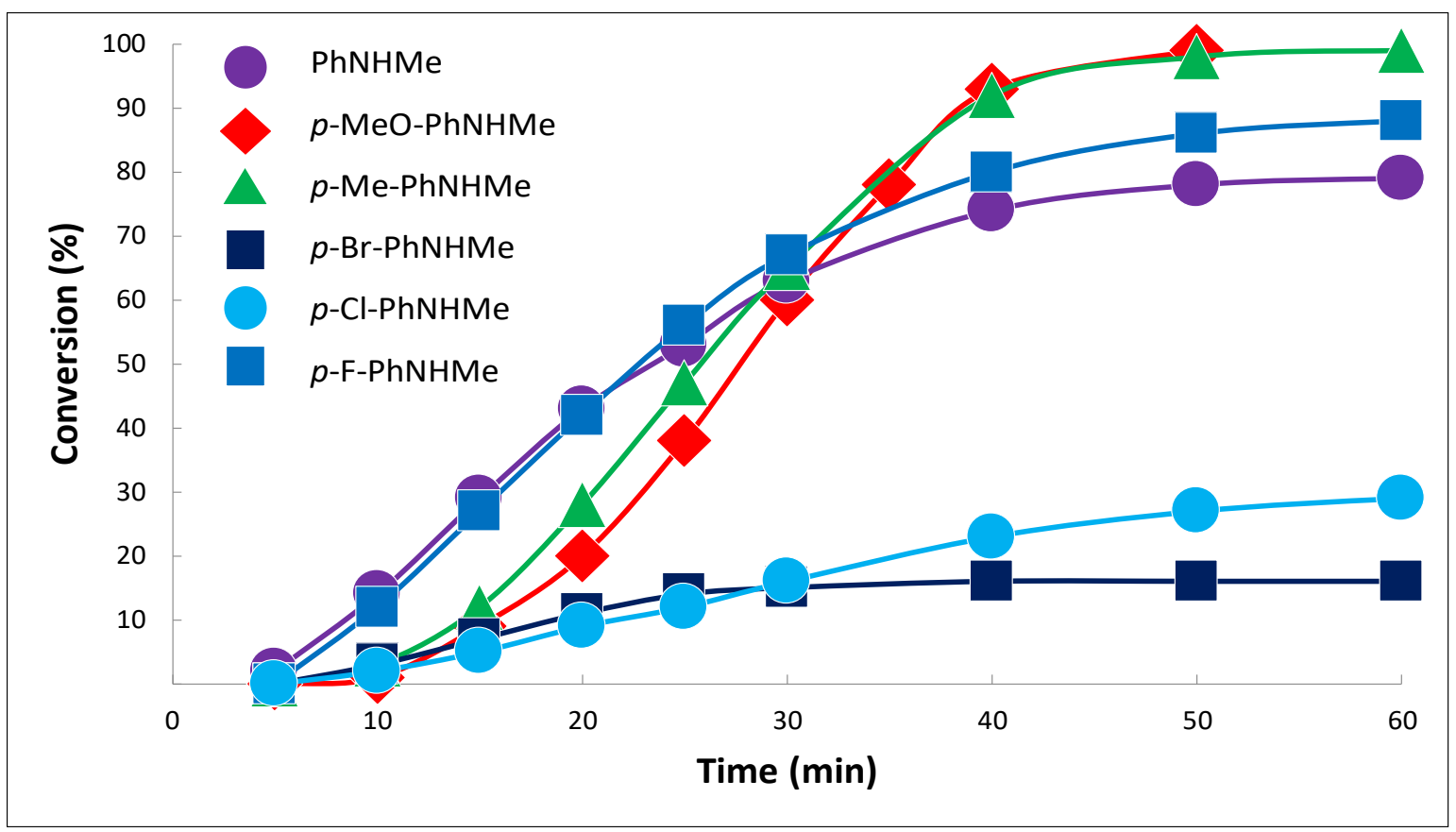

Figure S6. Conversion vs time plot for $p$-R-PhNHMe.

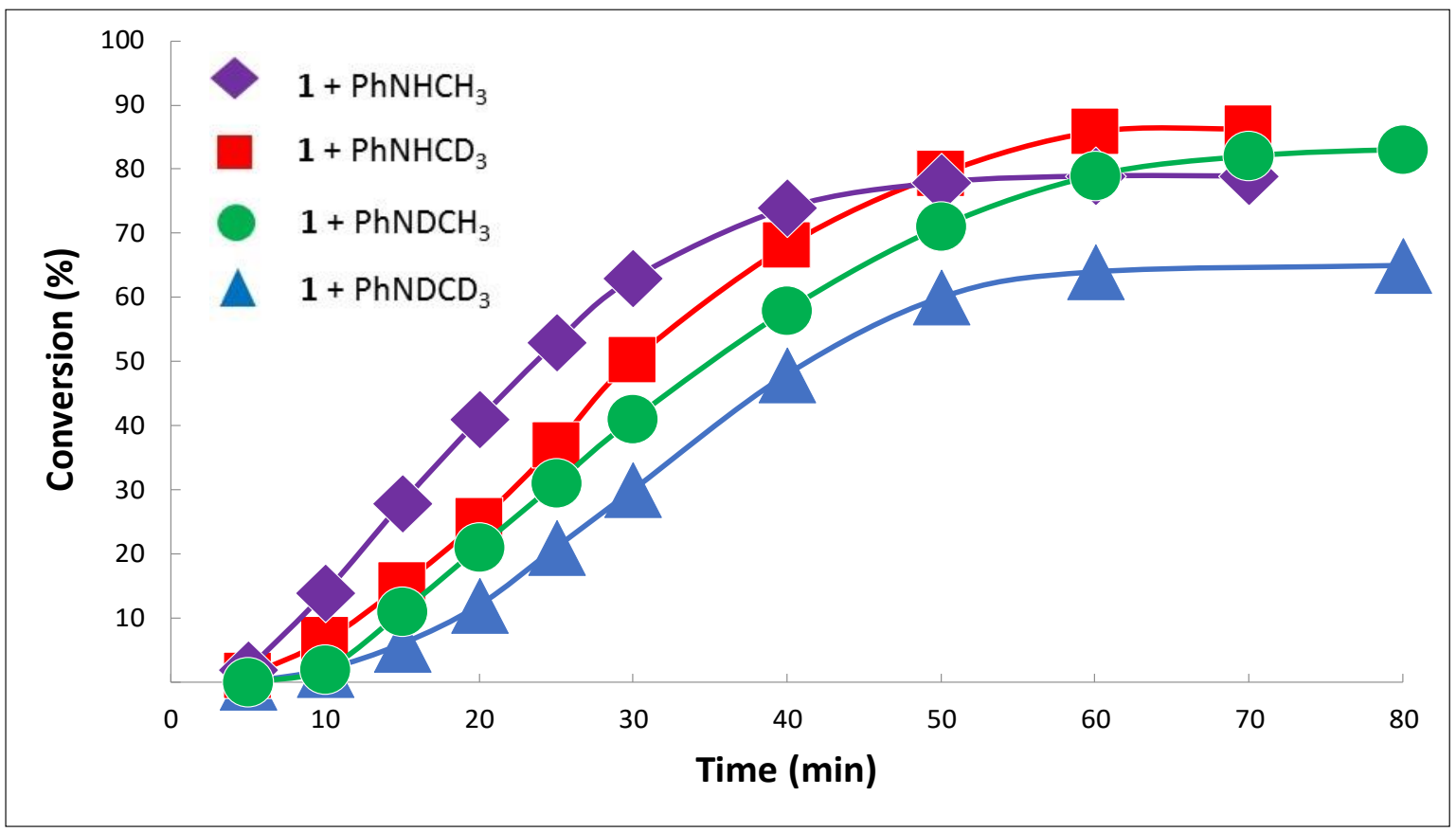

Figure S7. Conversion vs time plot for the KIE studies. 


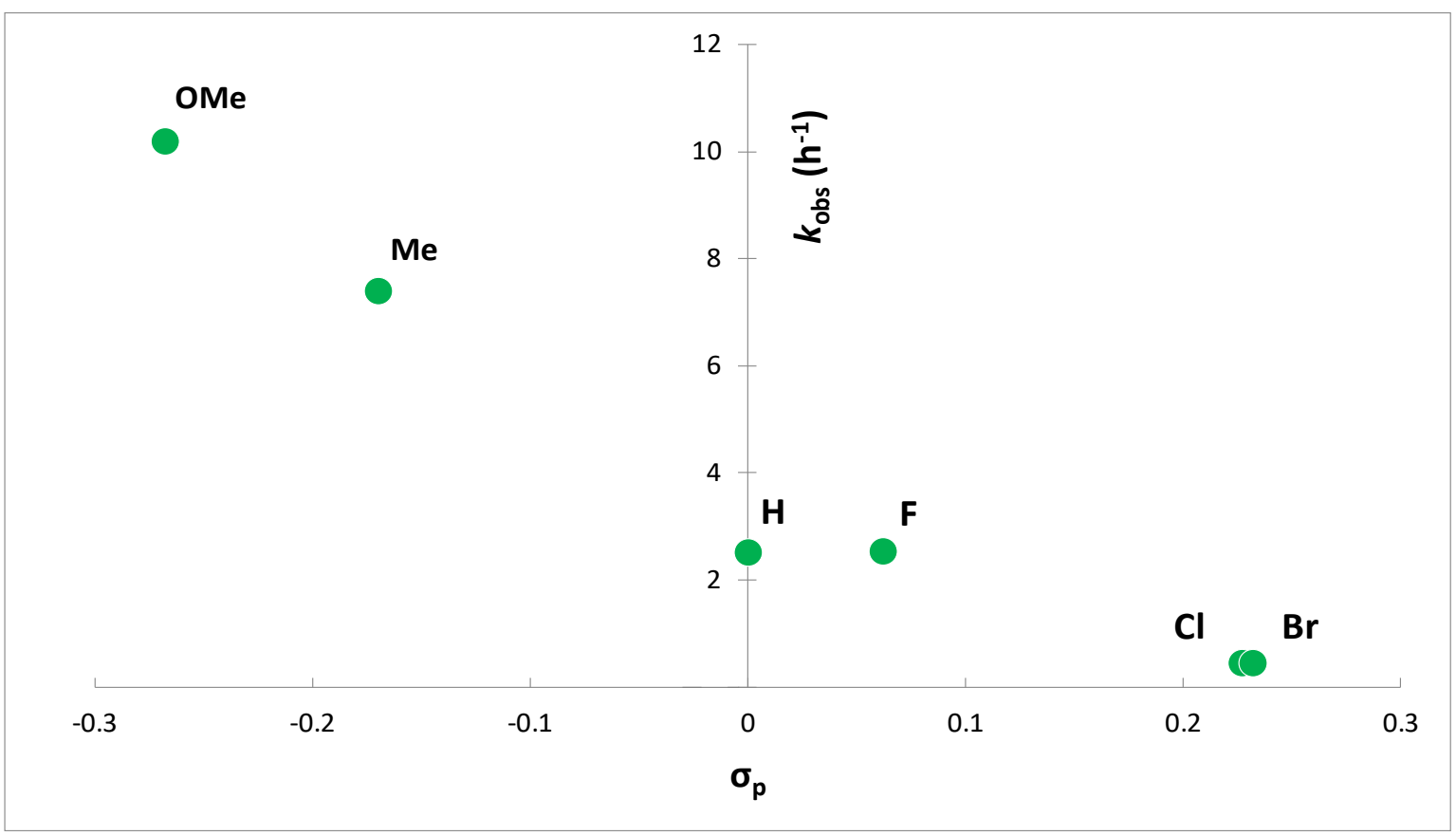

Figure S8. Hammett plot for the reaction of $p$-R-PhNHMe where $\mathrm{R}=\mathrm{OMe}, \mathrm{Me}, \mathrm{H}, \mathrm{F}, \mathrm{Cl}$ and $\mathrm{Br}$. 


\section{S4. General procedure for the amines and alkenes scope}

Unless otherwise stated, a mixture of amine $(0.5 \mathrm{mmol})$ and alkene $(0.5 \mathrm{mmol})$ substrates in $d_{8}$-toluene $(0.3 \mathrm{~g})$ were mixed with $\mathrm{L} 2 \mathrm{Ta}\left(\mathrm{CH}_{2} \mathrm{SiMe}_{3}\right)_{3} \mathrm{Cl}(5 \mu \mathrm{mol})$ and transferred into a J-Young NMR tube. All vials were further rinsed with $d_{8}$-toluene $(0.2$ $\mathrm{g}$ ) and the resulting solution was added to the J-Young NMR tube. An initial ${ }^{1} \mathrm{H}$ NMR spectrum was recorded, and the sample was added to a pre-heated oil bath at $110^{\circ} \mathrm{C}$ for 3 hours. The hydroaminoalkylation products were purified by silica gel chromatography, eluent: hexanes: EtOAc: $\mathrm{NEt}_{3}=5: 4: 1$, unless otherwise stated.

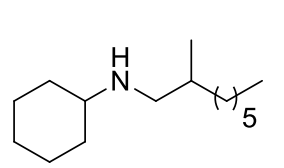

$\mathrm{N}$-(2-methyloctyl)cyclohexanamine, (3): $\mathrm{N}$-methylcyclohexanamine (56 mg, $0.5 \mathrm{mmol}$ ), 1-octene (56 mg, $0.5 \mathrm{mmol}), \mathrm{L} 2 \mathrm{Ta}\left(\mathrm{CH}_{2} \mathrm{SiMe}_{3}\right)_{3} \mathrm{Cl}(3 \mathrm{mg}, 0.005 \mathrm{mmol})$. Yield $(92 \mathrm{mg}, 82 \%) .{ }^{1} \mathrm{H} \mathrm{NMR}\left(\mathrm{CDCl}_{3}\right.$, $400 \mathrm{MHz}, 298 \mathrm{~K}): \delta$ 2.59-2.49 $(\mathrm{m}, 1 \mathrm{H}, \mathrm{NC}(H) \mathrm{H}), 2.44-2.29(\mathrm{~m}, 2 \mathrm{H}, \mathrm{NC}(\mathrm{H}) \mathrm{H}$ and $\mathrm{CH})$, 1.94-1.81 (m, $\left.2 \mathrm{H}, \mathrm{CH}_{2}\right), 1.78-1.66\left(\mathrm{~m}, 2 \mathrm{H}, \mathrm{CH}_{2}\right), 1.66-1.51\left(\mathrm{~m}, 2 \mathrm{H}, \mathrm{CH}_{2}\right), 1.41-0.99$ (overlapping $\mathrm{m}, 16 \mathrm{H}, \mathrm{CH}_{2}$ ), 0.92-0.83 (m, 6H, CH$)$ ppm. ${ }^{13} \mathrm{C} \mathrm{NMR}\left(\mathrm{CDCl}_{3}, 75 \mathrm{MHz}, 298 \mathrm{~K}\right): \delta 57.1(\mathrm{CH}), 53.8\left(\mathrm{CH}_{2}\right), 35.3\left(\mathrm{CH}_{2}\right)$, 33.6 $\left(\mathrm{CH}_{2}\right), 32.1\left(\mathrm{CH}_{2}\right), 29.8\left(\mathrm{CH}_{2}\right), 27.1\left(\mathrm{CH}_{2}\right), 26.4\left(\mathrm{CH}_{2}\right), 25.3\left(\mathrm{CH}_{2}\right), 22.8\left(\mathrm{CH}_{2}\right), 18.4\left(\mathrm{CH}_{3}\right), 14.3(\mathrm{CH}) \mathrm{ppm}$. HRMS (ESI): $\mathrm{m} / \mathrm{z}$ calcd for $\mathrm{C}_{15} \mathrm{H}_{32} \mathrm{~N}\left[\mathrm{M}+\mathrm{H}^{+}\right]: 226.2534$. Found: 226.2532.

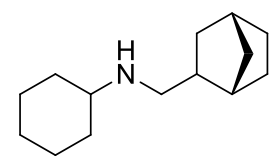

$\mathrm{N}$-(bicyclo[2.2.1] heptan-2-ylmethyl)cyclohexanamine, (4): $\mathrm{N}$-methylcyclohexylamine (56 mg, 0.5 $\mathrm{mmol}$ ), norbornene (56 mg, $0.5 \mathrm{mmol})$, L2Ta $\left(\mathrm{CH}_{2} \mathrm{SiMe}_{3}\right)_{3} \mathrm{Cl}$ (3 mg, $\left.0.005 \mathrm{mmol}\right)$. Yield (93 mg, 90\%). ${ }^{1} \mathrm{H}$ NMR $\left(\mathrm{CDCl}_{3}, 400 \mathrm{MHz}, 298 \mathrm{~K}\right): \delta 2.55\left(\mathrm{dt}, \mathrm{J}_{\mathrm{H}-\mathrm{H}}=10.5,4.0 \mathrm{~Hz}, 1 \mathrm{H}, \mathrm{C}(\mathrm{H}) \mathrm{H}\right), 2.44-2.30(\mathrm{~m}, 2 \mathrm{H}$, $\mathrm{CH}_{2}$ ), 2.19 (br s, $\left.1 \mathrm{H}, \mathrm{C}(H) \mathrm{H}\right), 2.05$ (br s, $\left.1 \mathrm{H}, \mathrm{C}(\mathrm{H}) H\right), 1.88\left(\mathrm{~d}, \mathrm{~J}_{\mathrm{H}-\mathrm{H}}=11.8 \mathrm{~Hz}, 2 \mathrm{H}, \mathrm{CH}_{2}\right)$, 1.78-1.34 (overlapping $\mathrm{m}, 9 \mathrm{H}, \mathrm{CH}$ and $\mathrm{CH}_{2}$ ), $1.33-0.94$ (overlapping $\mathrm{m}, 11 \mathrm{H}, \mathrm{CH}$ and $\mathrm{CH}_{2}$ ) ppm. ${ }^{13} \mathrm{C}$ NMR $\left(\mathrm{CDCl}_{3}, 100 \mathrm{MHz}, 298 \mathrm{~K}\right): \delta 57.2(\mathrm{NCH}), 52.8\left(\mathrm{NCH}_{2}\right), 42.6(\mathrm{CH}), 39.6(\mathrm{CH}), 36.5\left(\mathrm{CH}_{2}\right), 36.4(\mathrm{CH})$, $35.5\left(\mathrm{CH}_{2}\right)$, $33.6\left(\mathrm{CH}_{2}\right), 30.1\left(\mathrm{CH}_{2}\right), 29.0\left(\mathrm{CH}_{2}\right), 26.3\left(\mathrm{CH}_{2}\right), 25.3\left(\mathrm{CH}_{2}\right)$ ppm. HRMS $(\mathrm{ESI}): m / z$ calcd for $\mathrm{C}_{14} \mathrm{H}_{26} \mathrm{~N}\left[\mathrm{M}+\mathrm{H}^{+}\right]$: 208.2065. Found: 208.2066.<smiles>CCCCNCC(C)C</smiles>

$\mathrm{N}$-butyl-2-methyloctan-1-amine, (5): $\mathrm{N}$-methylbutylamine (56 mg, $0.5 \mathrm{mmol}), 1$-octene $(56 \mathrm{mg}$, $0.5 \mathrm{mmol})$, $\mathrm{L} 2 \mathrm{Ta}\left(\mathrm{CH}_{2} \mathrm{SiMe}_{3}\right)_{3} \mathrm{Cl}(15 \mathrm{mg}, 0.025 \mathrm{mmol})$. Reaction temperature: $145{ }^{\circ} \mathrm{C}$. Yield (76 mg, $68 \%) . \mathrm{R}_{\mathrm{f}} 0.18\left(20 \%\right.$ EtOAc in Hexanes with $\left.1 \% \mathrm{NEt}_{3}\right) .{ }^{1} \mathrm{H} \mathrm{NMR}\left(\mathrm{CDCl}_{3}, 400 \mathrm{MHz}, 298 \mathrm{~K}\right): \delta 2.55$ $\left(\mathrm{dt}, J_{\mathrm{H}-\mathrm{H}}=7.2,1.5 \mathrm{~Hz}, 2 \mathrm{H}, \mathrm{CH}_{2}\right), 2.48\left(\mathrm{dd}, J_{\mathrm{H}-\mathrm{H}}=11.3,5.9 \mathrm{~Hz}, \mathrm{NC}(H) \mathrm{HCH}\right), 2.33\left(\mathrm{dd}, J_{\mathrm{H}-\mathrm{H}}=11.7\right.$, $7.4 \mathrm{~Hz}, \mathrm{NC}(\mathrm{H}) \mathrm{HCH}$ ), 1.63-1.50 (m, 1H,CH), 1.37-1.16 (overlapping $\mathrm{m}, 13 \mathrm{H}, \mathrm{CH}_{2}$ ), 0.91-0.81 (overlapping $\mathrm{m}, 9 \mathrm{H}, \mathrm{CH}$ ) ppm. ${ }^{13} \mathrm{C}$ NMR $\left(\mathrm{CDCl}_{3}, 75 \mathrm{MHz}, 298 \mathrm{~K}\right): \delta 56.5\left(\mathrm{NCH}_{2}\right), 50.8\left(\mathrm{NCH}_{2}\right), 49.8\left(\mathrm{CH}_{2}\right), 35.2\left(\mathrm{CH}_{2}\right), 32.9(\mathrm{CH}), 32.0\left(\mathrm{CH}_{2}\right), 29.7\left(\mathrm{CH}_{2}\right)$, $27.0\left(\mathrm{CH}_{2}\right)$, $22.8\left(\mathrm{CH}_{2}\right), 20.6\left(\mathrm{CH}_{2}\right), 18.2\left(\mathrm{CH}_{3}\right), 14.2\left(\mathrm{CH}_{3}\right), 14.1\left(\mathrm{CH}_{3}\right) \cdot \mathrm{ppm}$. HRMS (ESI): $m / z$ calcd for $\mathrm{C}_{13} \mathrm{H}_{30} \mathrm{~N}\left[\mathrm{M}+\mathrm{H}^{+}\right]$: 200.2378. Found: 200.2377.<smiles>CCCCNCC1CC2CCC1C2</smiles>

N-bicyclo[2.2.1]heptan-2-ylmethyl)butan-1-amine, (6): $\mathrm{N}$-methylbutylamine (44 mg, $0.5 \mathrm{mmol}$ ), norbornene (56 mg, $0.5 \mathrm{mmol})$, L2Ta $\left(\mathrm{CH}_{2} \mathrm{SiMe}_{3}\right)_{3} \mathrm{Cl}(3 \mathrm{mg}, 0.025 \mathrm{mmol})$. Reaction temperature:

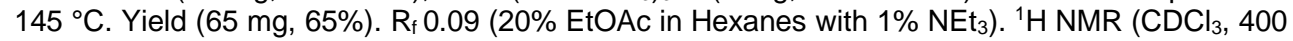
$\mathrm{MHz}, 298 \mathrm{~K}): \delta 2.60-2.52\left(\mathrm{~m}, 2 \mathrm{H}, \mathrm{CH}_{2}\right), 2.45\left(\mathrm{dd}, \mathrm{J}_{\mathrm{H}-\mathrm{H}}=11.6,8.3 \mathrm{~Hz}, \mathrm{NC}(H) \mathrm{HCH}\right), 2.29\left(\mathrm{dd}, \mathrm{J}_{\mathrm{H}-\mathrm{H}}\right.$ $=11.5,7.0 \mathrm{~Hz}, \mathrm{NC}(\mathrm{H}) H \mathrm{CH}), 2.18(\mathrm{br} \mathrm{s}, 1 \mathrm{H}, \mathrm{C}(H) \mathrm{H}), 2.02(\mathrm{br} \mathrm{s}, 1 \mathrm{H}, \mathrm{C}(\mathrm{H}) H), 1.63-1.53(\mathrm{~m}, 1 \mathrm{H}, \mathrm{CH})$, 1.53-1.21 (overlapping $\mathrm{m}, 9 \mathrm{H}, \mathrm{CH}$ and $\mathrm{CH}_{2}$ ), $1.20-1.03$ (overlapping $\left.\mathrm{m}, 4 \mathrm{H}, \mathrm{CH}_{2}\right), 0.89\left(\mathrm{t}, \mathrm{J}_{\mathrm{H}-\mathrm{H}}=7.3 \mathrm{~Hz}, 3 \mathrm{H}, \mathrm{CH}_{3}\right) \mathrm{ppm} .{ }^{13} \mathrm{C} \mathrm{NMR}\left(\mathrm{CDCl}_{3}, 75 \mathrm{MHz}\right.$, $298 \mathrm{~K})$ : $\delta 55.1\left(\mathrm{NCH}_{2}\right), 50.9\left(\mathrm{NCH}_{2}\right), 49.5\left(\mathrm{CH}_{2}\right.$ or $\left.\mathrm{CH}\right), 41.6\left(\mathrm{CH}_{2}\right.$ or $\left.\mathrm{CH}\right), 39.6\left(\mathrm{CH}_{2}\right.$ or $\left.\mathrm{CH}\right), 36.43(\mathrm{CH}$ or $\mathrm{CH}), 36.38(\mathrm{CH}$ or $\mathrm{CH}), 35.5\left(\mathrm{CH}_{2}\right.$ or $\left.\mathrm{CH}\right), 31.2\left(\mathrm{CH}_{2}\right.$ or $\left.\mathrm{CH}\right), 30.0\left(\mathrm{CH}_{2}\right), 28.9\left(\mathrm{CH}_{2}\right), 20.6\left(\mathrm{CH}_{2}\right), 14.0\left(\mathrm{CH}_{3}\right)$.ppm. HRMS (ESI): $\mathrm{m} / z \mathrm{calcd}$ for $\mathrm{C}_{12} \mathrm{H}_{24} \mathrm{~N}\left[\mathrm{M}+\mathrm{H}^{+}\right]:$182.1908. Found: 182.1912.

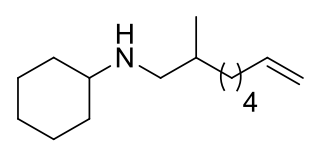

N-(2-methyloct-7-en-1-yl)cyclohexanamine, (7): N-methylcyclohexanamine (56 mg, $0.5 \mathrm{mmol})$, 1,7-octadiene (110 mg, $1 \mathrm{mmol})$, L2Ta $\left(\mathrm{CH}_{2} \mathrm{SiMe}_{3}\right)_{3} \mathrm{Cl}$ (3 $\left.\mathrm{mg}, 0.005 \mathrm{mmol}\right)$. Yield (100 mg, 60\%). ${ }^{1} \mathrm{H}$ NMR $\left(\mathrm{CDCl}_{3}, 400 \mathrm{MHz}, 298 \mathrm{~K}\right): \delta 5.80$ (ddt, $\mathrm{J}=17.1,10.3,6.7,1.3 \mathrm{~Hz}, 1 \mathrm{H}, \mathrm{CH}=\mathrm{CH}_{2}$ ), 5.03$4.88\left(\mathrm{~m}, 2 \mathrm{H}, \mathrm{CH}=\mathrm{CH}_{2}\right), 2.59-2.48(\mathrm{~m}, 1 \mathrm{H}, \mathrm{NC}(H) \mathrm{H}), 2.44-2.29(\mathrm{~m}, 2 \mathrm{H}, \mathrm{NC}(\mathrm{H}) \mathrm{H}$ and $\mathrm{CH}), 2.04(\mathrm{q}$, $\left.J_{\mathrm{H}-\mathrm{H}}=6.4 \mathrm{~Hz}, \mathrm{CH}_{2}\right), 1.93-1.82\left(\mathrm{~m},, 2 \mathrm{H}, \mathrm{CH}_{2}\right), 1.77-1.66\left(\mathrm{~m}, 2 \mathrm{H}, \mathrm{CH}_{2}\right), 1.65-1.51\left(\mathrm{~m}, 2 \mathrm{H}, \mathrm{CH}_{2}\right)$, 1.44-0.97 (overlapping $\left.\mathrm{m}, 13 \mathrm{H}, \mathrm{CH}_{2}\right), 0.88\left(\mathrm{~d}, \mathrm{~J}_{\mathrm{H}-\mathrm{H}}=6.5 \mathrm{~Hz}, 3 \mathrm{H}, \mathrm{CH}_{3}\right) \mathrm{ppm} .{ }^{13} \mathrm{C} \mathrm{NMR}\left(\mathrm{CDCl}_{3}, 75 \mathrm{MHz}, 298 \mathrm{~K}\right): \delta 139.3$ $\left(\mathrm{CH}=\mathrm{CH}_{2}\right), 114.3\left(\mathrm{CH}=\mathrm{CH}_{2}\right), 57.1\left(\mathrm{CH}_{2}\right), 53.7(\mathrm{CH}), 35.1\left(\mathrm{CH}_{2}\right), 33.9\left(\mathrm{CH}_{2}\right), 33.6\left(\mathrm{CH}_{2}\right), 29.3\left(\mathrm{CH}_{2}\right), 26.6\left(\mathrm{CH}_{2}\right), 26.4\left(\mathrm{CH}_{2}\right)$, $25.3\left(\mathrm{CH}_{2}\right), 18.4\left(\mathrm{CH}_{3}\right)$ ppm. HRMS (ESI): $\mathrm{m} / z$ calcd for $\mathrm{C}_{15} \mathrm{H}_{30} \mathrm{~N}\left[\mathrm{M}+\mathrm{H}^{+}\right]: 224.2378$. Found: 224.2382.

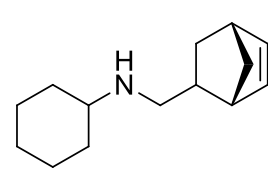

N-bicyclo[2.2.1]hept-5-en-2-ylmethyl)cyclohexanamine, (8): $\mathrm{N}$-methylcyclohexanamine (56 mg, $0.5 \mathrm{mmol}$ ), norbornadiene (46 mg, $0.5 \mathrm{mmol})$, L2Ta $\left(\mathrm{CH}_{2} \mathrm{SiMe}_{3}\right)_{3} \mathrm{Cl}(3 \mathrm{mg}, 0.005 \mathrm{mmol})$. Yield (45 $\mathrm{mg}, 44 \%) .{ }^{1} \mathrm{H} \mathrm{NMR}\left(\mathrm{CDCl}_{3}, 400 \mathrm{MHz}, 298 \mathrm{~K}\right): \delta 6.10-6.00$ (overlapping $\left.\mathrm{m}, 2 \mathrm{H}, \mathrm{CH}=\mathrm{CH}\right), 2.78(\mathrm{~s}, 1 \mathrm{H}$, $\mathrm{CH}$ ), 2.69-2.57 (overlapping $\mathrm{m}, 3 \mathrm{H}, \mathrm{CH}$ and $\left.\mathrm{CH}_{2}\right), 2.44-2.34(\mathrm{~m}, 1 \mathrm{H}, \mathrm{CH}), 1.91-1.81\left(\mathrm{~m}, 2 \mathrm{H}, \mathrm{CH}_{2}\right)$, 1.76-1.66 (m, 2H, CH$), 1.64-1.56(\mathrm{~m}, 1 \mathrm{H}, \mathrm{CH}), 1.55-1.46(\mathrm{~m}, 1 \mathrm{H}, \mathrm{CH}), 1.34-1.00$ (overlapping $\mathrm{m}$, $10 \mathrm{H}, \mathrm{CH}$ and $\left.\mathrm{CH}_{2}\right)$ ppm. ${ }^{13} \mathrm{C} \mathrm{NMR}\left(\mathrm{CDCl}_{3}, 75 \mathrm{MHz}, 298 \mathrm{~K}\right): \delta 136.8(\mathrm{CH}), 136.6(\mathrm{CH}), 57.2(\mathrm{CH})$, 53.0 $\left(\mathrm{CH}_{2}\right)$, $45.3\left(\mathrm{CH}_{2}\right), 44.8(\mathrm{CH}), 41.8(\mathrm{CH}), 39.6(\mathrm{CH}), 33.81\left(\mathrm{CH}_{2}\right), 33.78\left(\mathrm{CH}_{2}\right), 31.7\left(\mathrm{CH}_{2}\right), 26.3\left(\mathrm{CH}_{2}\right), 25.3\left(\mathrm{CH}_{2}\right) \mathrm{ppm}$. HRMS (ESI): $m / z$ calcd for $\mathrm{C}_{14} \mathrm{H}_{24} \mathrm{~N}\left[\mathrm{M}+\mathrm{H}^{+}\right]$: 206.1908. Found: 206.1907.

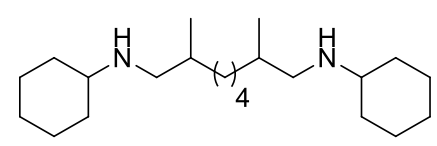

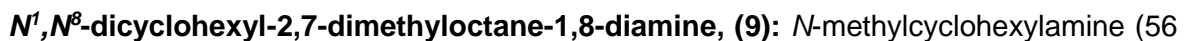
$\mathrm{mg}, 0.5 \mathrm{mmol})$, 1,7-octadiene (110 mg, $0.1 \mathrm{mmol})$, L2Ta $\left(\mathrm{CH}_{2} \mathrm{SiMe}_{3}\right)_{3} \mathrm{Cl}(15 \mathrm{mg}, 0.025$ $\mathrm{mmol})$. Yield (123 mg, $78 \%)$. ${ }^{1} \mathrm{H}$ NMR $\left(\mathrm{CDCl}_{3}, 400 \mathrm{MHz}, 298 \mathrm{~K}\right): \delta 2.57-2.47(\mathrm{~m}, 2 \mathrm{H}$, 
$\mathrm{NCH}), 2.41-2.28\left(\mathrm{~m}, 4 \mathrm{H}, \mathrm{NCH}_{2}\right), 1.86\left(\mathrm{~d}, \mathrm{~J}_{\mathrm{H}-\mathrm{H}}=12.0 \mathrm{~Hz}, 4 \mathrm{H}, \mathrm{CH}_{2}\right), 1.76-1.66\left(\mathrm{~m}, 4 \mathrm{H}, \mathrm{CH}_{2}\right), 1.65-1.47(\mathrm{~m}, 4 \mathrm{H}, \mathrm{CH}$ and $\mathrm{CH}$ ), $1.42-0.96\left(\mathrm{~m}, 2 \mathrm{H}, \mathrm{CH}_{2}\right), 0.87\left(\mathrm{~d}, \mathrm{~J}_{\mathrm{H}-\mathrm{H}}=6.6 \mathrm{~Hz}, 6 \mathrm{H}, \mathrm{CH}_{3}\right) \mathrm{ppm} .{ }^{13} \mathrm{C} \mathrm{NMR}\left(\mathrm{CDCl}_{3}, 100 \mathrm{MHz}, 298 \mathrm{~K}\right): \delta 57.1(\mathrm{NCH}), 53.8$ $\left(\mathrm{CH}_{2}\right)$, $53.7\left(\mathrm{CH}_{2}\right), 35.3\left(\mathrm{CH}_{2}\right)$, $33.9\left(\mathrm{CH}_{2}\right), 33.8\left(\mathrm{CH}_{2}\right), 33.6(\mathrm{CH}), 27.4\left(\mathrm{CH}_{2}\right), 26.4\left(\mathrm{CH}_{2}\right), 25.3\left(\mathrm{CH}_{2}\right), 18.4\left(\mathrm{CH}_{3}\right) \mathrm{ppm}$. HRMS (ESI): $m / z$ calcd for $\mathrm{C}_{22} \mathrm{H}_{45} \mathrm{~N}_{2}\left[\mathrm{M}+\mathrm{H}^{+}\right]$: 337.3582. Found: 337.3586 .

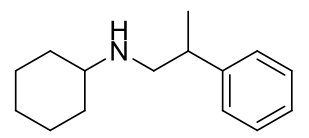

$\mathrm{N}$-(2-phenylpropyl)cyclohexanamine, (10): $\mathrm{N}$-methylcyclohexylamine (56 $\mathrm{mg}, 0.5 \mathrm{mmol}$ ), styrene (52 mg, $0.5 \mathrm{mmol})$, L2Ta $\left(\mathrm{CH}_{2} \mathrm{SiMe}_{3}\right)_{3} \mathrm{Cl}(3 \mathrm{mg}, 0.005 \mathrm{mmol})$. Yield $(21 \mathrm{mg}, 22 \%)$. ${ }^{1} \mathrm{H} \mathrm{NMR}$ $\left(\mathrm{CDCl}_{3}, 400 \mathrm{MHz}, 298 \mathrm{~K}\right): \delta$ 7.34-7.27 (m, 2H, ArH), 7.24-7.18 (m, 3H, $\left.\mathrm{ArH}\right), 2.98-2.86(\mathrm{~m}, 1 \mathrm{H}$, $\mathrm{CH}$, 2.87-2.73 (m, 2H, CH $\mathrm{CH}_{2}$, 2.43-2.31 (m, $\left.1 \mathrm{H}, \mathrm{CH}\right), 1.92-1.43$ (overlapping $\mathrm{m}, 7 \mathrm{H}, \mathrm{CH}$ and $\mathrm{CH}_{2}$ ), 1.32-0.92 (overlapping $\mathrm{m}, 9 \mathrm{H}, \mathrm{CH}_{2}$ and $\left.\mathrm{CH}_{3}\right) \mathrm{ppm} .{ }^{13} \mathrm{C} \mathrm{NMR}\left(\mathrm{CDCl}_{3}, 100 \mathrm{MHz}, 298 \mathrm{~K}\right): \delta 145.6,128.7,127.3,126.5($ all $\operatorname{ArH}), 56.9(\mathrm{NCH}), 54.2\left(\mathrm{NCH}_{2}\right), 40.2(\mathrm{CH}), 33.5\left(\mathrm{CH}_{2}\right), 26.3\left(\mathrm{CH}_{2}\right), 25.2\left(\mathrm{CH}_{2}\right), 20.4\left(\mathrm{CH}_{3}\right) \mathrm{ppm}$. HRMS (ESI): $\mathrm{m} / z \mathrm{calcd}$ for $\mathrm{C}_{15} \mathrm{H}_{24} \mathrm{~N}\left[\mathrm{M}+\mathrm{H}^{+}\right]: 218.1908$. Found: 218.1908.

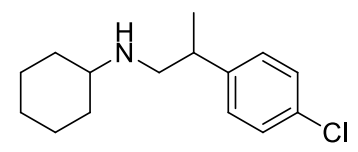

$\mathrm{N}$-(2-(4-chlorophenyl)propyl)cyclohexanamine, (11): $\mathrm{N}$-methylcyclohexylamine $(56 \mathrm{mg}, 0.5$ $\mathrm{mmol}$ ), 4-chlorostyrene ( $69 \mathrm{mg}, 0.5 \mathrm{mmol}), \mathrm{L} 2 \mathrm{Ta}\left(\mathrm{CH}_{2} \mathrm{SiMe}_{3}\right)_{3} \mathrm{Cl}(3 \mathrm{mg}, 0.005 \mathrm{mmol})$. Yield (15 $\mathrm{mg}, 12 \%) .{ }^{1} \mathrm{H}$ NMR $\left(\mathrm{CDCl}_{3}, 400 \mathrm{MHz}, 298 \mathrm{~K}\right): \delta$ 7.31-7.23 (m, 2H, $\left.\mathrm{ArH}\right), 7.19-7.11(\mathrm{~m}, 3 \mathrm{H}$, $\mathrm{ArH}), 2.96-2.84(\mathrm{~m}, 1 \mathrm{H}, \mathrm{CH}), 2.84-2.68\left(\mathrm{~m}, 2 \mathrm{H}, \mathrm{CH}_{2}\right), 2.42-2.30(\mathrm{~m}, 1 \mathrm{H}, \mathrm{CH}), 1.90-1.35$ (overlapping $\mathrm{m}, 7 \mathrm{H}, \mathrm{CH}$ and $\mathrm{CH}_{2}$ ), 1.33-0.91 (overlapping $\mathrm{m}, 9 \mathrm{H}, \mathrm{CH}_{2}$ and $\mathrm{CH}_{3}$ ) ppm. ${ }^{13} \mathrm{C} \mathrm{NMR}$ $\left(\mathrm{CDCl}_{3}, 100 \mathrm{MHz}, 298 \mathrm{~K}\right): \delta$ 144.1, 132.1, 128.8, 128.7 (all ArH), $56.9(\mathrm{NCH}), 54.0\left(\mathrm{NCH}_{2}\right), 39.8(\mathrm{CH}), 33.5(\mathrm{CH}), 26.2$ $\left(\mathrm{CH}_{2}\right), 25.2\left(\mathrm{CH}_{2}\right), 20.4\left(\mathrm{CH}_{3}\right)$ ppm. HRMS (ESI): $\mathrm{m} / z$ calcd for $\mathrm{C}_{15} \mathrm{H}_{23} \mathrm{CIN}\left[\mathrm{M}+\mathrm{H}^{+}\right]: 252.1519$. Found: 252.1526.<smiles>C[Si](C)(C)CCCNC1CCCCC1</smiles>

$\mathbf{N}$-(3-(trimethylsilyl)propyl)cyclohexanamine, (12): $\mathrm{N}$-methylcyclohexanamine $(56 \mathrm{mg}, 0.5$ $\mathrm{mmol})$, vinylsilane (45 mg, $0.5 \mathrm{mmol}), \mathrm{L} 2 \mathrm{Ta}\left(\mathrm{CH}_{2} \mathrm{SiMe}_{3}\right)_{3} \mathrm{Cl}(3 \mathrm{mg}, 0.005 \mathrm{mmol})$. Yield $(64 \mathrm{mg}$, $64 \%)$. NMR and GC data show that the other regioisomer ( $\mathrm{N}-(2$ (trimethylsilyl)propyl)cyclohexanamine) is also present $(\sim 10 \%)$, however only the resonances of the main regiosiomer will be shown herein. ${ }^{1} \mathrm{H}$ NMR $\left(\mathrm{CDCl}_{3}, 400 \mathrm{MHz}, 298 \mathrm{~K}\right): \delta 2.62-2.54(\mathrm{~m}, 2 \mathrm{H}$, $\left.\mathrm{NCH}_{2}\right), 2.45-2.33(\mathrm{~m}, 1 \mathrm{H}, \mathrm{NCH}), 1.93-1.78\left(\mathrm{~m}, 2 \mathrm{H}, \mathrm{CH}_{2}\right), 1.78-1.66\left(\mathrm{~m}, 2 \mathrm{H}, \mathrm{CH}_{2}\right), 1.65-1.55(\mathrm{~m}, 2 \mathrm{H}, \mathrm{CH}), 1.51-1.39(\mathrm{~m}, 2 \mathrm{H}$, $\left.\mathrm{CH}_{2}\right), 1.32-0.98\left(\mathrm{~m}, 6 \mathrm{H}, \mathrm{CH}_{2}\right), 0.49-0.42\left(\mathrm{~m}, 2 \mathrm{H}, \mathrm{CH}_{2} \mathrm{Si}\right), 0.03(\mathrm{~s}, 9 \mathrm{H}, \mathrm{SiCH})_{3}$ ppm. ${ }^{13} \mathrm{C} \mathrm{NMR}\left(\mathrm{CDCl}_{3}, 100 \mathrm{MHz}, 298 \mathrm{~K}\right): \delta$ $57.0(\mathrm{NCH}), 50.5\left(\mathrm{NCH}_{2}\right)$, 33.8 $\left(\mathrm{CH}_{2}\right)$, $26.3\left(\mathrm{CH}_{2}\right), 25.3\left(\mathrm{CH}_{2}\right), 25.0\left(\mathrm{CH}_{2}\right), 14.4\left(\mathrm{CH}_{2}\right),-1.6(\mathrm{SiCH})$. ppm. HRMS $(\mathrm{ESI}): \mathrm{m} / \mathrm{z}$ calcd for $\mathrm{C}_{12} \mathrm{H}_{27} \mathrm{NSi}\left[\mathrm{M}+\mathrm{H}^{+}\right]: 214.1991$. Found: 214.1991 .

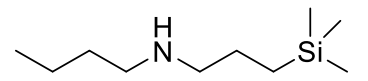

$\mathrm{N}$-(3-(trimethylsilyl)propyl)butan-1-amine, (13): $\mathrm{N}$-methylbutylamine (43 $\mathrm{mg}, 0.5 \mathrm{mmol}$ ), vinylsilane (45 mg, $0.5 \mathrm{mmol})$, $\mathrm{L} 2 \mathrm{Ta}\left(\mathrm{CH}_{2} \mathrm{SiMe}_{3}\right)_{3} \mathrm{Cl}$ (15 mg, $\left.0.025 \mathrm{mmol}\right)$. Yield (15 mg, $30 \%$ ). NMR and GC data show that both regioisomers are present in an approximate ration of 3:1(linear:branched). The chemical shifts corresponding to the main regioisomer will be presented herein. ${ }^{1} \mathrm{H} \mathrm{NMR}\left(\mathrm{CDCl} \mathrm{C}_{3}\right.$, $400 \mathrm{MHz}, 298 \mathrm{~K}$ ): $\delta$ 2.66-2.56 (m, $\left.2 \mathrm{H}, \mathrm{CH}_{2}\right), 1.57-1.45$ (overlapping $\mathrm{m}, 3 \mathrm{H}, \mathrm{CH}$ and $\left.\mathrm{CH}_{2}\right), 1.42-1.28\left(\mathrm{~m}, 2 \mathrm{H}, \mathrm{CH}_{2}\right), 0.92\left(\mathrm{t}, \mathrm{J}_{\mathrm{H}}\right.$ $\left.H=7.3 \mathrm{~Hz}, 3 \mathrm{H}, \mathrm{CH}_{3}\right), 0.51-0.45\left(\mathrm{~m}, 2 \mathrm{H}, \mathrm{CH}_{2} \mathrm{Si}\right), 0.02\left(\mathrm{~s}, 9 \mathrm{H}, \mathrm{SiCH}_{3}\right) \mathrm{ppm} .{ }^{13} \mathrm{C} \mathrm{NMR}\left(\mathrm{CDCl}_{3}, 100 \mathrm{MHz}, 298 \mathrm{~K}\right): \delta 53.3\left(\mathrm{CH}_{2}\right)$, $49.6\left(\mathrm{CH}_{2}\right), 32.0\left(\mathrm{CH}_{2}\right), 24.2\left(\mathrm{CH}_{2}\right), 20.6\left(\mathrm{CH}_{2}\right), 14.3\left(\mathrm{CH}_{2}\right), 14.2\left(\mathrm{CH}_{3}\right),-1.6(\mathrm{SiCH}) \mathrm{ppm}$. HRMS (ESI): $\mathrm{m} / z \mathrm{calcd}$ for $\mathrm{C}_{10} \mathrm{H}_{26} \mathrm{NSi}\left[\mathrm{M}+\mathrm{H}^{+}\right]:$188.1834. Found: 188.1836.

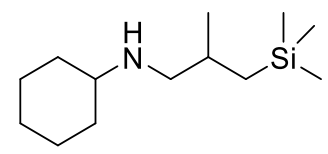

$\mathrm{N}$-(2-methyl-3-(trimethylsilyl)propyl)cyclohexanamine, (14): $\mathrm{N}$-methylcyclohexylamine (57 mg, $0.5 \mathrm{mmol}$ ), allylsilane (86 mg, $0.75 \mathrm{mmol}), \mathrm{L} 2 \mathrm{Ta}\left(\mathrm{CH}_{2} \mathrm{SiMe}_{3}\right)_{3} \mathrm{Cl}(15 \mathrm{mg}, 0.025 \mathrm{mmol}) .14$ was purified by chromatography on silica gel $\left(1-2 \% \mathrm{MeOH}\right.$ in DCM with $\left.1 \% \mathrm{NEt}_{3}\right)$. Yield $(72 \mathrm{mg}, 63$ \%). $\mathrm{R}_{\mathrm{f}} 0.23\left(5 \% \mathrm{MeOH}\right.$ in DCM with $\left.1 \% \mathrm{NEt}_{3}\right) .{ }^{1} \mathrm{H} \mathrm{NMR}\left(\mathrm{CDCl}_{3}, 300 \mathrm{MHz}, 298 \mathrm{~K}\right): \delta 2.60-2.33(\mathrm{~m}$, $\left.3 \mathrm{H}, \mathrm{CH}_{2}+\mathrm{NCH}\right), 2.03-1.50\left(\mathrm{~m}, 6 \mathrm{H}, \mathrm{CH}_{2}\right), 1.32-1.02\left(\mathrm{~m}, 5 \mathrm{H}, \mathrm{CH}_{2}+\mathrm{CH}\right), 0.94\left(\mathrm{~d}, \mathrm{~J}_{\mathrm{H}-\mathrm{H}}=6.6 \mathrm{~Hz}\right.$, $\left.\left.3 \mathrm{H}, \mathrm{CH}_{3}\right), 0.65\left(\mathrm{dd}, \mathrm{J}_{\mathrm{H}-\mathrm{H}}=14.6,4.6 \mathrm{~Hz}, 1 \mathrm{H}, \mathrm{CH}_{2} \mathrm{Si}\right), 0.36\left(\mathrm{dd}, \mathrm{J}_{\mathrm{H}-\mathrm{H}}=14.6,9.3 \mathrm{~Hz}, 1 \mathrm{H}, \mathrm{CH}_{2} \mathrm{Si}\right),-0.00(\mathrm{~s}, 9 \mathrm{H}, \mathrm{SiCH})\right) \mathrm{ppm} .{ }^{13} \mathrm{C}$ $\operatorname{NMR}\left(\mathrm{CDCl}_{3}, 75 \mathrm{MHz}, 298 \mathrm{~K}\right): \delta 57.1(\mathrm{NCH}), 56.4\left(\mathrm{NCH}_{2}\right), 33.4\left(\mathrm{CH}_{2}\right), 30.0(\mathrm{CH}), 26.2\left(\mathrm{CH}_{2}\right), 25.3\left(\mathrm{CH}_{2}\right), 23.2\left(\mathrm{CH}_{2}\right), 21.2$ $\left(\mathrm{CH}_{3}\right),-0.4\left(\mathrm{SiCH}_{3}\right)$ ppm. HRMS (ESI): $\mathrm{m} / z$ calcd for $\mathrm{C}_{13} \mathrm{H}_{30} \mathrm{NSi}\left[\mathrm{M}+\mathrm{H}^{+}\right]$: 228.2148. Found: 228.2143.<smiles>CCCCC(CNC1CCCCC1)C[Si](C)(C)C</smiles>

$\mathrm{N}$-(2-((trimethylsilyl)methyl)hexyl)cyclohexanamine, (15): $\mathrm{N}$-methylcyclohexylamine (57 mg, 0.5 $\mathrm{mmol}$ ), hexenyltrimethylsilane (117 mg, $0.75 \mathrm{mmol}), \mathrm{L} 2 \mathrm{Ta}\left(\mathrm{CH}_{2} \mathrm{SiMe}_{3}\right)_{3} \mathrm{Cl}(15 \mathrm{mg}, 0.025 \mathrm{mmol}) .15$ was purified by chromatography on silica gel $\left(1-2 \% \mathrm{MeOH}\right.$ in DCM with $\left.1 \% \mathrm{NEt}_{3}\right)$. Yield (58 mg, 43 \%). $\mathrm{R}_{\mathrm{f}} 0.34\left(5 \% \mathrm{MeOH}\right.$ in $\mathrm{DCM}$ with $\left.1 \% \mathrm{NEt}_{3}\right) .{ }^{1} \mathrm{H} \mathrm{NMR}\left(\mathrm{CDCl}_{3}, 300 \mathrm{MHz}, 298 \mathrm{~K}\right): \delta 2.63-2.30$ (m, $\left.3 \mathrm{H}, \mathrm{CH}_{2}+\mathrm{CH}\right), 2.00-1.79\left(\mathrm{~m}, 2 \mathrm{H}, \mathrm{CH}_{2}\right), 1.79-1.51\left(\mathrm{~m}, 4 \mathrm{H}, \mathrm{CH}_{2}\right), 1.41-0.99\left(\mathrm{~m}, 13 \mathrm{H}, \mathrm{CH}_{2}\right), 0.89(\mathrm{t}$, $\left.J_{\mathrm{H}-\mathrm{H}}=6.6 \mathrm{~Hz}, 3 \mathrm{H}, \mathrm{CH}_{3}\right), 0.53\left(\mathrm{dd}, \mathrm{J}_{\mathrm{H}-\mathrm{H}}=6.6,1.4 \mathrm{~Hz}, 2 \mathrm{H}, \mathrm{CH}_{2} \mathrm{Si}\right), 0.01\left(\mathrm{~s}, 9 \mathrm{H}, \mathrm{SiCH}_{3}\right) \mathrm{ppm} .{ }^{13} \mathrm{C} \mathrm{NMR}$ $\left(\mathrm{CDCl}_{3}, 75 \mathrm{MHz}, 298 \mathrm{~K}\right)$ : $\delta 57.2(\mathrm{NCH}), 53.2\left(\mathrm{NCH}_{2}\right), 34.8\left(\mathrm{CH}_{2}\right), 33.56\left(\mathrm{CH}_{2}\right.$ or $\left.\mathrm{CH}\right), 33.51\left(\mathrm{CH}_{2}\right.$ or $\mathrm{CH}), 28.8\left(\mathrm{CH}_{2}\right), 26.3\left(\mathrm{CH}_{2}\right), 25.3\left(\mathrm{CH}_{2}\right), 23.2\left(\mathrm{CH}_{2}\right), 20.4\left(\mathrm{CH}_{2} \mathrm{Si}\right), 14.3\left(\mathrm{CH}_{3}\right),-0.4\left(\mathrm{SiCH}_{3}\right) \mathrm{ppm}$. HRMS (ESI): $m / z$ calcd for $\mathrm{C}_{16} \mathrm{H}_{36} \mathrm{NSi}\left[\mathrm{M}+\mathrm{H}^{+}\right]: 270.2617$. Found: 270.2615 .<smiles>COc1ccc(NC(C)C(C)(C)CC(C)(C)C)cc1</smiles>

4-methoxy- $N$-(3-methylnonan-2-yl)aniline, (16): $N$-ethyl-4-methoxyaniline (76 mg, 0.5 mmol), 1-octene (84 mg, $0.75 \mathrm{mmol}), \mathrm{L} 2 \mathrm{Ta}\left(\mathrm{CH}_{2} \mathrm{SiMe} 3\right)_{3} \mathrm{Cl}(15 \mathrm{mg}, 0.025 \mathrm{mmol}) .16$ was purified by chromatography on silica gel (10\% EtOAc in Hexanes with $\left.1 \% \mathrm{NEt}_{3}\right)$. Yield (68 mg, $52 \%) . R_{f} 0.62$ (20\% EtOAc in Hezanes with $\left.1 \% \mathrm{NEt}_{3}\right) .{ }^{1} \mathrm{H} \mathrm{NMR}\left(\mathrm{CDCl}_{3}, 300 \mathrm{MHz}, 298\right.$ $\mathrm{K}): \delta 6.76\left(\mathrm{~d}, J_{\mathrm{H}-\mathrm{H}}=8.9 \mathrm{~Hz}, 2 \mathrm{H}, \mathrm{Ar}\right), 6.56\left(\mathrm{~d}, \mathrm{~J}_{\mathrm{H}-\mathrm{H}}=8.8 \mathrm{~Hz}, 2 \mathrm{H}, \mathrm{Ar}\right), 3.74\left(\mathrm{~s}, 3 \mathrm{H}, \mathrm{OCH}_{3}\right), 3.33$ (qd, $\left.J_{H-H}=6.5,4.4 \mathrm{~Hz}, 1 \mathrm{H}, \mathrm{NCH}\right), 1.71-1.54(\mathrm{~m}, 1 \mathrm{H}, \mathrm{CH}), 1.49-1.11\left(\mathrm{~m}, 10 \mathrm{H}, \mathrm{CH}_{2}\right), 1.08(\mathrm{~d}$, $\left.J_{\mathrm{H}-\mathrm{H}}=6.5 \mathrm{~Hz}, 3 \mathrm{H}, \mathrm{CH}_{3}\right), 0.93\left(\mathrm{~d}, \mathrm{~J}_{\mathrm{H}-\mathrm{H}}=6.9 \mathrm{~Hz}, 3 \mathrm{H}, \mathrm{CH}_{3}\right), 0.87\left(\mathrm{t}, J_{\mathrm{H}-\mathrm{H}}=6.6 \mathrm{~Hz}, 3 \mathrm{H}, \mathrm{CH}_{2} \mathrm{CH}_{3}\right) \mathrm{ppm} .{ }^{13} \mathrm{C} \mathrm{NMR}\left(\mathrm{CDCl}_{3}, 101\right.$ 
$\mathrm{MHz}, 298 \mathrm{~K}): \delta 152.0$ (Ar, HMBC), 141.3 (Ar, HMBC), 115.08 (2C, HSQC, Ar), $55.98\left(\mathrm{OCH}_{3}\right), 54.5$ (HSQC, NCH), 37.22 $\left(\mathrm{CH}_{2}\right)$, $32.08\left(\mathrm{CH}_{2}\right)$, $32.02\left(\mathrm{CH}_{2}\right), 29.77\left(\mathrm{CH}_{2}\right), 27.62\left(\mathrm{CH}_{2}\right), 22.81\left(\mathrm{CH}_{2}\right), 17.0\left(\mathrm{CH}_{3}\right), 16.0\left(\mathrm{CH}_{3}\right), 14.2\left(\mathrm{CH}_{3}\right)$ ppm. HRMS (ESI): $\mathrm{m} / z$ calcd for $\mathrm{C}_{17} \mathrm{H}_{30} \mathrm{NO}\left[\mathrm{M}+\mathrm{H}^{+}\right]: 264.2327$. Found: 264.2325 .

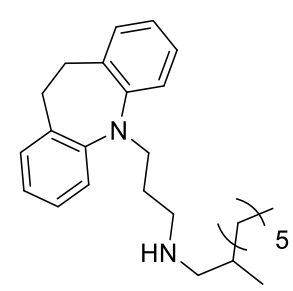

Desipramine-N-(2-methyl)octylamine, (19): Desipramine (60 mg, $0.25 \mathrm{mmol}), 1$-octene (28 mg, $0.25 \mathrm{mmol})$, $\mathrm{L} 2 \mathrm{Ta}\left(\mathrm{CH}_{2} \mathrm{SiMe}_{3}\right)_{3} \mathrm{Cl}(7 \mathrm{mg}, 0.01 \mathrm{mmol})$. 19 was purified by chromatography on silica gel $\left(1-2 \% \mathrm{MeOH}\right.$ in $\mathrm{DCM}$ with $\left.1 \% \mathrm{NEt}_{3}\right)$. Yield $(71 \mathrm{mg}, 75 \%) .{ }^{1} \mathrm{H} \mathrm{NMR}\left(\mathrm{CDCl}_{3}, 300 \mathrm{MHz}, 298 \mathrm{~K}\right): \delta$ 7.20-6.99 (m, 6H), 6.99-6.81 (m, 2H), 3.89-3.71 (m, 2H), 3.13 (s, 4H), 2.99-2.79 (m, 2H), 2.57 (dd, $\left.J_{\mathrm{H}-\mathrm{H}}=12.4,5.4 \mathrm{~Hz}, 1 \mathrm{H}\right), 2.41\left(\mathrm{dd}, J_{\mathrm{H}-\mathrm{H}}=12.3,8.6 \mathrm{~Hz}, 1 \mathrm{H}\right), 2.16-1.94(\mathrm{~m}, 2 \mathrm{H}), 1.81-1.62(\mathrm{~m}, 1 \mathrm{H})$, $1.39-1.05(\mathrm{~m}, 11 \mathrm{H}), 0.98-0.72(\mathrm{~m}, 6 \mathrm{H}) \mathrm{ppm} .{ }^{13} \mathrm{C} \mathrm{NMR}\left(\mathrm{CDCl}_{3}, 75 \mathrm{MHz}, 298 \mathrm{~K}\right): \delta 147.7(\mathrm{Ar}), 134.2$ (Ar), $130.1(\mathrm{Ar}), 126.7(\mathrm{Ar}), 123.1(\mathrm{Ar}), 119.9(\mathrm{Ar}), 53.3\left(\mathrm{NCH}_{2}\right), 47.8\left(\mathrm{NCH}_{2}\right), 45.8\left(\mathrm{CH}_{2}\right), 34.7\left(\mathrm{CH}_{2}\right)$, $32.3\left(\mathrm{ArCH}_{2}\right), 31.9(\mathrm{CH}), 30.6\left(\mathrm{CH}_{2}\right), 29.9\left(\mathrm{CH}_{2}\right), 29.5\left(\mathrm{CH}_{2}\right), 26.6\left(\mathrm{CH}_{2}\right), 24.0\left(\mathrm{CH}_{2}\right), 22.8\left(\mathrm{CH}_{2}\right)$, $17.9\left(\mathrm{CH}_{3}\right), 14.2\left(\mathrm{CH}_{3}\right)$ ppm. HRMS (ESI): $\mathrm{m} / \mathrm{z}$ calcd for $\mathrm{C}_{26} \mathrm{H}_{39} \mathrm{~N}_{2}\left[\mathrm{M}+\mathrm{H}^{+}\right]$: 379.3113 . Found:

379.3117.

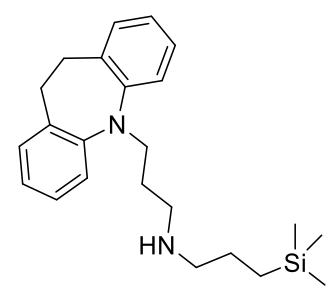

Desipramine- $\mathrm{N}$-(3-(trimethylsilyl)propylamine, (20): Desipramine (60 $\mathrm{mg}, 0.25 \mathrm{mmol}$ ), vinylsilane $(25 \mathrm{mg}, 0.25 \mathrm{mmol}), \mathrm{L} 2 \mathrm{Ta}\left(\mathrm{CH}_{2} \mathrm{SiMe}_{3}\right)_{3} \mathrm{Cl}(7 \mathrm{mg}, 0.01 \mathrm{mmol}) .20$ was purified by chromatography on silica gel (1-2\% MeOH in DCM with $\left.1 \% \mathrm{NEt}_{3}\right)$. Yield (65 mg, $74 \%$ ). NMR data shows that both regioisomers are present in an approximate ration of 7:3 (linear:branched). However only the resonances of the main regiosiomer will be shown herein. ${ }^{1} \mathrm{H} \mathrm{NMR}\left(\mathrm{CDCl}_{3}, 400\right.$ $\mathrm{MHz}, 298 \mathrm{~K}): \delta 7.18-6.98(\mathrm{~m}, 6 \mathrm{H}, \mathrm{Ar}), 6.92\left(\mathrm{t}, J_{\mathrm{H}-\mathrm{H}}=7.3 \mathrm{~Hz}, 2 \mathrm{H}, \mathrm{Ar}\right), 3.82\left(\mathrm{t}, J_{\mathrm{H}-\mathrm{H}}=6.4 \mathrm{~Hz}, 1 \mathrm{H}\right.$, $\left.\mathrm{NCH}_{2}\right), 3.13\left(\mathrm{~s}, 1 \mathrm{H}, \mathrm{ArCH}_{2}\right), 3.00-2.78\left(\mathrm{~m}, 2 \mathrm{H}, \mathrm{CH}_{2}\right), 2.70-2.61\left(\mathrm{~m}, 2 \mathrm{H}, \mathrm{CH}_{2}\right), 2.17-1.98(\mathrm{~m}, 2 \mathrm{H}$, $\left.\mathrm{CH}_{2}\right), 1.74-1.53\left(\mathrm{~m}, 2 \mathrm{H}, \mathrm{CH}_{2}\right), 0.51-0.30\left(\mathrm{~m}, 2 \mathrm{H}, \mathrm{CH}_{2}\right),-0.04\left(\mathrm{~s}, 9 \mathrm{H}, \mathrm{SiCH}_{3}\right)$ ppm. ${ }^{13} \mathrm{C} \mathrm{NMR}$ $\left(\mathrm{CDCl}_{3}, 101 \mathrm{MHz}, 298 \mathrm{~K}\right)$ : $\delta 147.8(\mathrm{Ar}), 134.2(\mathrm{Ar}), 130.1(\mathrm{Ar}), 126.7(\mathrm{Ar}), 123.1$ (Ar), $119.9(\mathrm{Ar})$, $50.3\left(\mathrm{NCH}_{2}\right), 47.9\left(\mathrm{NCH}_{2}\right), 45.4\left(\mathrm{CH}_{2}\right)$, $32.3\left(\mathrm{ArCH}_{2}\right), 24.4\left(\mathrm{CH}_{2}\right), 20.9\left(\mathrm{CH}_{2}\right), 13.8\left(\mathrm{CH}_{2}\right),-1.6(\mathrm{SiCH}) \mathrm{ppm}$. HRMS $(\mathrm{ESI}): \mathrm{m} / \mathrm{z}$ calcd for $\mathrm{C}_{23} \mathrm{H}_{34} \mathrm{~N}_{2} \mathrm{Si}\left[\mathrm{M}+\mathrm{H}^{+}\right]: 367.2564$. Found: 367.2567.<smiles>CC(C)(C)CNCCC(Oc1ccc(C(F)(F)F)cc1)c1ccccc1</smiles>

Fluoxetine- $\mathrm{N}$-(2-methyl)octylamine, (22): Fluoxetine (77 mg, $0.25 \mathrm{mmol}), 1$-octene (28 mg, $0.25 \mathrm{mmol}), \mathrm{L} 2 \mathrm{Ta}\left(\mathrm{CH}_{2} \mathrm{SiMe}_{3}\right)_{3} \mathrm{Cl}(7 \mathrm{mg}, 0.01 \mathrm{mmol}) .22$ was purified by chromatography on silica gel (1-2\% MeOH in DCM with $\left.1 \% \mathrm{NEt}_{3}\right)$. Yield $(48 \mathrm{mg}, 45 \%)$. $\mathrm{R}_{\mathrm{f}} 0.45\left(5 \% \mathrm{MeOH}\right.$ in DCM with $\left.1 \% \mathrm{NEt}_{3}\right){ }^{1} \mathrm{H} \mathrm{NMR}\left(\mathrm{CDCl}_{3}, 300 \mathrm{MHz}, 298 \mathrm{~K}\right): \delta 7.42$ (d, $\left.J_{\mathrm{H}-\mathrm{H}}=8.7 \mathrm{~Hz}, 2 \mathrm{H}, \mathrm{Ar}\right), 7.39-7.21(\mathrm{~m}, 5 \mathrm{H}, \mathrm{Ar}), 6.90\left(\mathrm{~d}, \mathrm{~J}_{\mathrm{H}-\mathrm{H}}=8.7 \mathrm{~Hz}, 2 \mathrm{H}, \mathrm{Ar}\right), 5.42-5.23$ $(\mathrm{m}, 1 \mathrm{H}, \mathrm{OCH}), 2.80\left(\mathrm{t}, J_{\mathrm{H}-\mathrm{H}}=6.9 \mathrm{~Hz}, 2 \mathrm{H}, \mathrm{CH}_{2}\right), 2.55\left(\mathrm{dd}, J_{\mathrm{H}-\mathrm{H}}=11.6,5.7 \mathrm{~Hz}, 1 \mathrm{H}, \mathrm{CH}_{2}\right)$, $2.40\left(\mathrm{dd}, J_{\mathrm{H}-\mathrm{H}}=11.7,7.5 \mathrm{~Hz}, 1 \mathrm{H}, \mathrm{CH}_{2}\right), 2.24\left(\mathrm{dt}, \mathrm{J}_{\mathrm{H}-\mathrm{H}}=14.6,7.3 \mathrm{~Hz}, 1 \mathrm{H}, \mathrm{CH}_{2}\right), 2.09\left(\mathrm{dt}, J_{\mathrm{H}-\mathrm{H}}=13.7,6.9 \mathrm{~Hz}, 1 \mathrm{H}, \mathrm{CH}\right.$ ), 1.62 (br s, $1 \mathrm{H}, \mathrm{CH}), 1.39-1.01\left(\mathrm{~m}, 10 \mathrm{H}, \mathrm{CH}_{2}\right), 0.88\left(\mathrm{t}, \mathrm{J}_{\mathrm{H}-\mathrm{H}}=7.0 \mathrm{~Hz}, 6 \mathrm{H}, \mathrm{CH}_{3}\right) \mathrm{ppm} .{ }^{13} \mathrm{C} \mathrm{NMR}\left(\mathrm{CDCl}_{3}, 75 \mathrm{MHz}, 298 \mathrm{~K}\right): \delta 160.64$ $(\mathrm{Ar}), 141.01(\mathrm{Ar}), 128.94(\mathrm{Ar}), 128.02(\mathrm{Ar}), 126.90\left(\mathrm{q}, \mathrm{J}_{\mathrm{C}-\mathrm{F}}=3.8 \mathrm{~Hz}, C_{3}\right), 126.30(\mathrm{Ar}), 125.96(\mathrm{Ar}), 125.72(\mathrm{Ar}), 115.91(\mathrm{Ar})$, $78.85(\mathrm{OCH}), 56.35\left(\mathrm{CH}_{2}\right), 46.27\left(\mathrm{CH}_{2}\right), 38.39\left(\mathrm{CH}_{2}\right), 35.04\left(\mathrm{CH}_{2}\right), 32.95(\mathrm{CH}), 32.02\left(\mathrm{CH}_{2}\right), 29.73\left(\mathrm{CH}_{2}\right), 27.02\left(\mathrm{CH}_{2}\right), 22.80$ $\left(\mathrm{CH}_{2}\right)$, $18.3\left(\mathrm{CH}_{3}\right), 14.2\left(\mathrm{CH}_{3}\right) \mathrm{ppm} .{ }^{19} \mathrm{~F} \mathrm{NMR}\left(\mathrm{CDCl}_{3}, 282 \mathrm{MHz}, 298 \mathrm{~K}\right): \delta-62.0 \mathrm{ppm}$. HRMS (ESI): $m / z$ calcd for $\mathrm{C}_{25} \mathrm{H}_{35} \mathrm{~F}_{3} \mathrm{NO}$ $\left[\mathrm{M}+\mathrm{H}^{+}\right]:$422.2670. Found: 422.2670 .

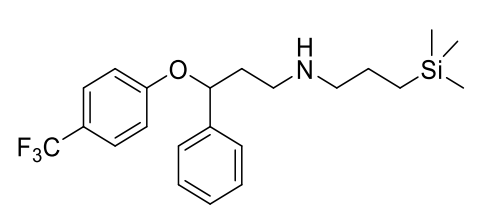

Fluoxetine-N-(3-(trimethylsilyl)propylamine, (23): Fluoxetine (77 mg, $0.25 \mathrm{mmol})$, vinylsilane (25 mg, $0.25 \mathrm{mmol})$, $\mathrm{L} 2 \mathrm{Ta}\left(\mathrm{CH}_{2} \mathrm{SiMe}_{3}\right)_{3} \mathrm{Cl}(7 \mathrm{mg}, 0.01 \mathrm{mmol})$. Conversion of $46 \%$ by HPLC-MS, 10 to $90 \%$ acetonitrile $[0.1 \%$ formic acid (FA)] in water $(0.1 \% \mathrm{FA})$ over $10 \mathrm{~min}$ followed by $90 \%$ acetonitrile $(0.1 \% \mathrm{FA})$ in water $(0.1 \% \mathrm{FA})$ over $5 \mathrm{~min}$, flow rate of $1 \mathrm{~mL} / \mathrm{min}$ on an Eclipse XDB-C18, $5 \mu \mathrm{m}, 4.6 \times 150 \mathrm{~mm}, \lambda=263 \mathrm{~nm}$, (Fluoxetine, $t_{R}=7.42 \mathrm{~min}$, area $\left.=54 \%,[\mathrm{M}+\mathrm{H}]^{+}=310\right),\left(23, \mathrm{t}_{\mathrm{R}}=10.11 \mathrm{~min}\right.$, area $=$ $\left.46 \%,[\mathrm{M}+\mathrm{H}]^{+}=410\right) .23$ was purified by chromatography on silica gel $(1-2 \% \mathrm{MeOH}$ in $\mathrm{DCM}$ with $1 \% \mathrm{NEt}_{3}$ ). NMR data shows that both regioisomers are present in an approximate ration of 7:3 (linear:branched) and contamination with $\mathbf{L} 2 \mathrm{H}$. Only the resonances of the main regiosiomer of 23 will be shown herein. ${ }^{1} \mathrm{H} \mathrm{NMR}\left(\mathrm{CDCl}{ }_{3}, 300\right.$ $\mathrm{MHz}, 298 \mathrm{~K}): \delta 7.44\left(\mathrm{~d}, J_{\mathrm{H}-\mathrm{H}}=8.7 \mathrm{~Hz}, 2 \mathrm{H}, \mathrm{Ar}\right), 7.38-7.24(\mathrm{~m}, 5 \mathrm{H}, \mathrm{Ar}), 6.92\left(\mathrm{~d}, J_{\mathrm{H}-\mathrm{H}}=8.7 \mathrm{~Hz}, 2 \mathrm{H}, \mathrm{Ar}\right), 5.42-5.29(\mathrm{~m}, 1 \mathrm{H}$, $\mathrm{OCH}), 4.50(\mathrm{br} \mathrm{s}, 1 \mathrm{H}, \mathrm{NH}), 2.82\left(\mathrm{t}, \mathrm{J}_{\mathrm{H}-\mathrm{H}}=6.9 \mathrm{~Hz}, 2 \mathrm{H}, \mathrm{NCH}\right), 2.63\left(\mathrm{t}, \mathrm{J}_{\mathrm{H}-\mathrm{H}}=7.5 \mathrm{~Hz}, 2 \mathrm{H}, \mathrm{NCH} \mathrm{H}_{2}\right), 2.33-2.00(\mathrm{~m}, 2 \mathrm{H}, \mathrm{CH}$ ), $1.61-1.40\left(\mathrm{~m}, 2 \mathrm{H}, \mathrm{CH}_{2}\right), 0.55-0.40\left(\mathrm{~m}, 2 \mathrm{H}, \mathrm{CH}_{2}\right),-0.01\left(\mathrm{~s}, 9 \mathrm{H}, \mathrm{SiCH}_{3}\right) \mathrm{ppm} .{ }^{13} \mathrm{C} \mathrm{NMR}\left(\mathrm{CDCl}_{3}, 100 \mathrm{MHz}, 298 \mathrm{~K}\right): \delta 160.6$

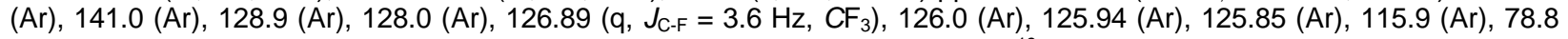
$(\mathrm{OCH}), 53.1\left(\mathrm{CH}_{2}\right), 45.9\left(\mathrm{CH}_{2}\right), 38.6\left(\mathrm{CH}_{2}\right), 24.1\left(\mathrm{CH}_{2}\right), 14.2\left(\mathrm{CH}_{2}\right),-1.6\left(\mathrm{SiCH}_{3}\right){ }^{19}{ }^{1} \mathrm{~F} \mathrm{NMR}\left(\mathrm{CDCl}_{3}, 282 \mathrm{MHz}, 298 \mathrm{~K}\right): \delta-61.6$ ppm. HRMS (ESI): $\mathrm{m} / \mathrm{z}$ calcd for $\mathrm{C}_{22} \mathrm{H}_{31} \mathrm{~F}_{3} \mathrm{NO}\left[\mathrm{M}+\mathrm{H}^{+}\right]$: 410.2127 . Found: 410.2123. 


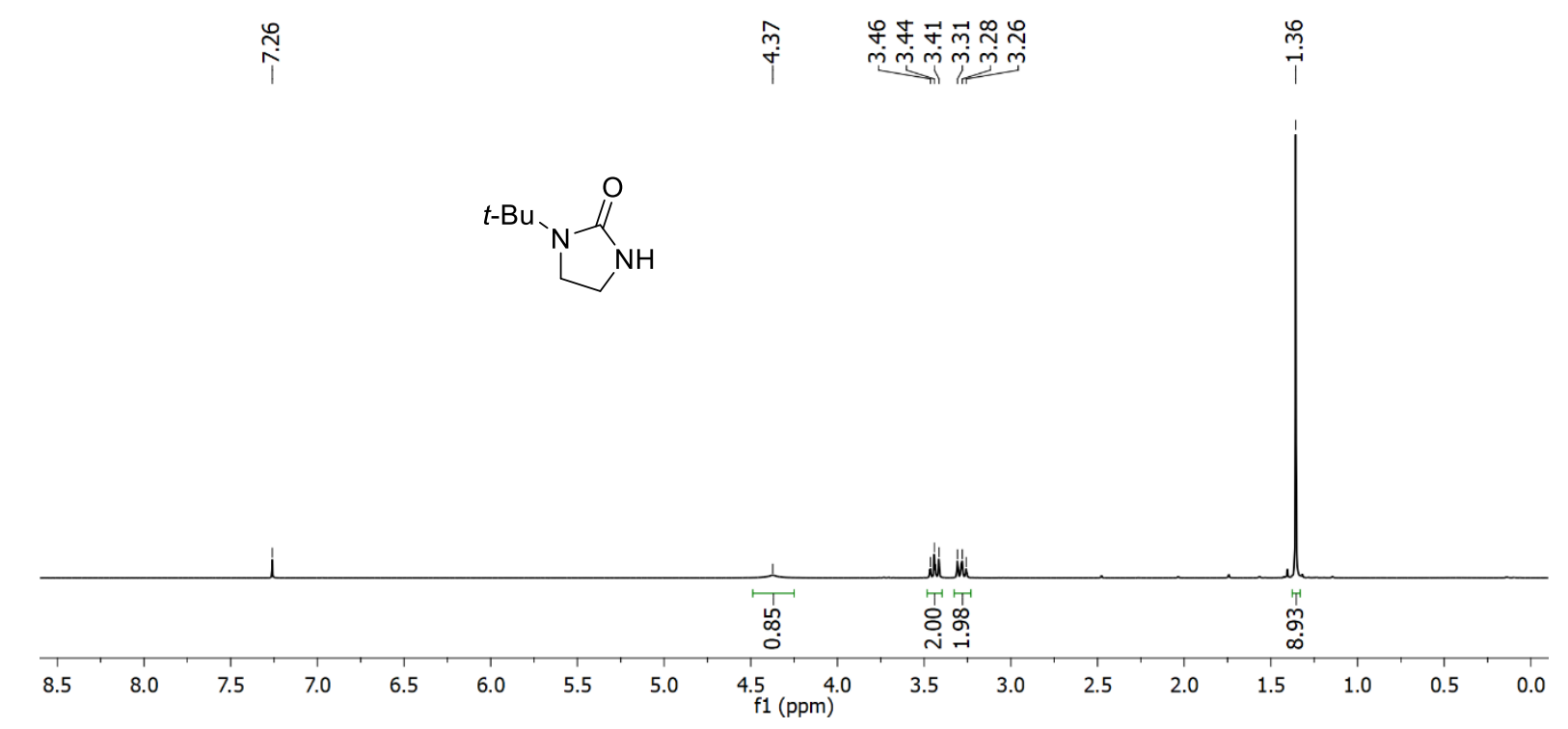

Figure S9. ${ }^{1} \mathrm{H}$ NMR spectrum $\left(300 \mathrm{MHz}, \mathrm{CDCl}_{3}, 298 \mathrm{~K}\right)$ of 1-(tert-butyl)imidazolidin-2-one (L2H).

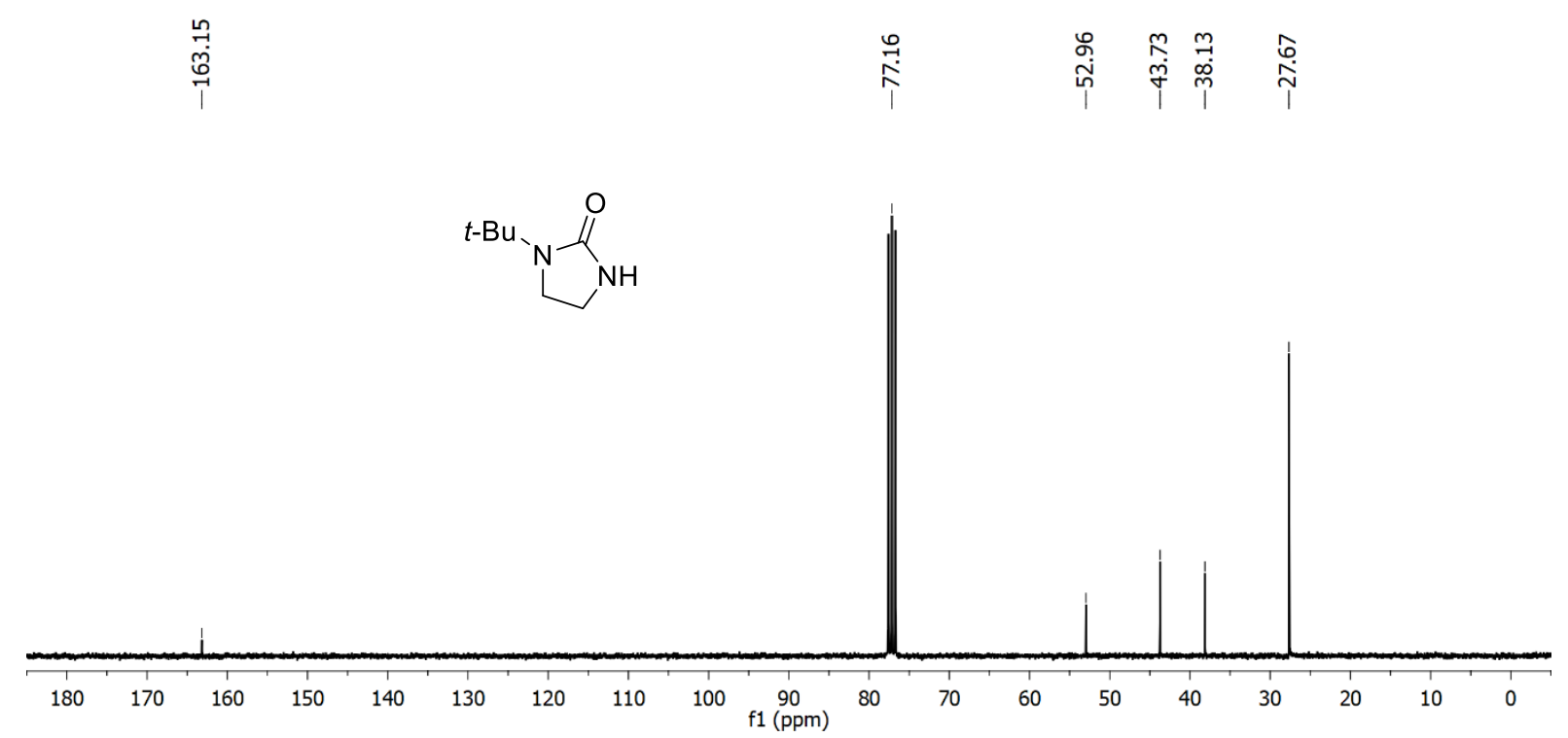

Figure S10. ${ }^{13} \mathrm{C}$ NMR spectrum $\left(75 \mathrm{MHz}, \mathrm{CDCl}_{3}, 298 \mathrm{~K}\right)$ of 1-(tert-butyl)imidazolidin-2-one (L2H). 


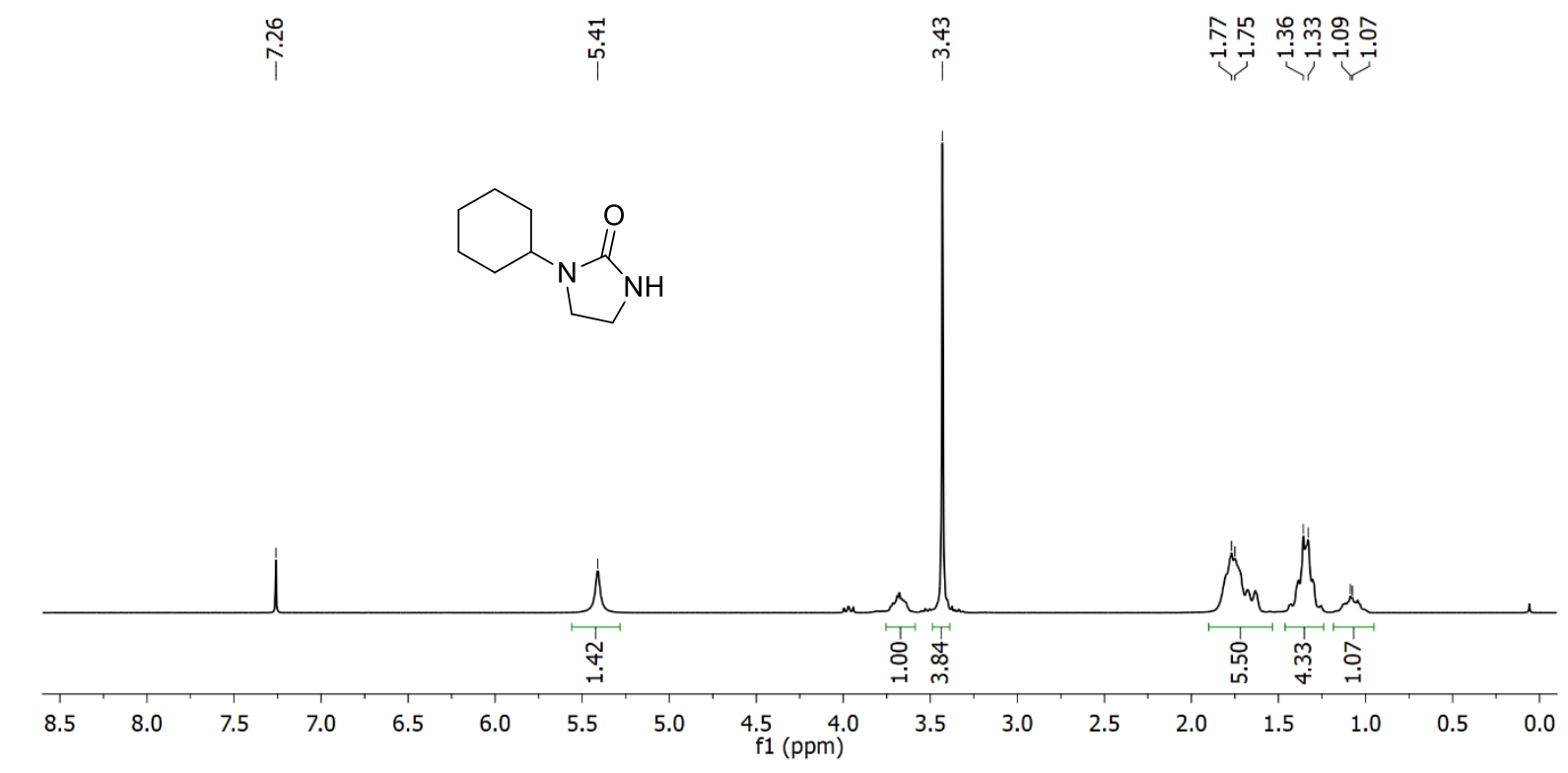

Figure S11. ${ }^{1} \mathrm{H}$ NMR spectrum (300 MHz, $\mathrm{CDCl}_{3}, 298 \mathrm{~K}$ ) of 1-cyclohexylimidazolidin-2-one (L3H).

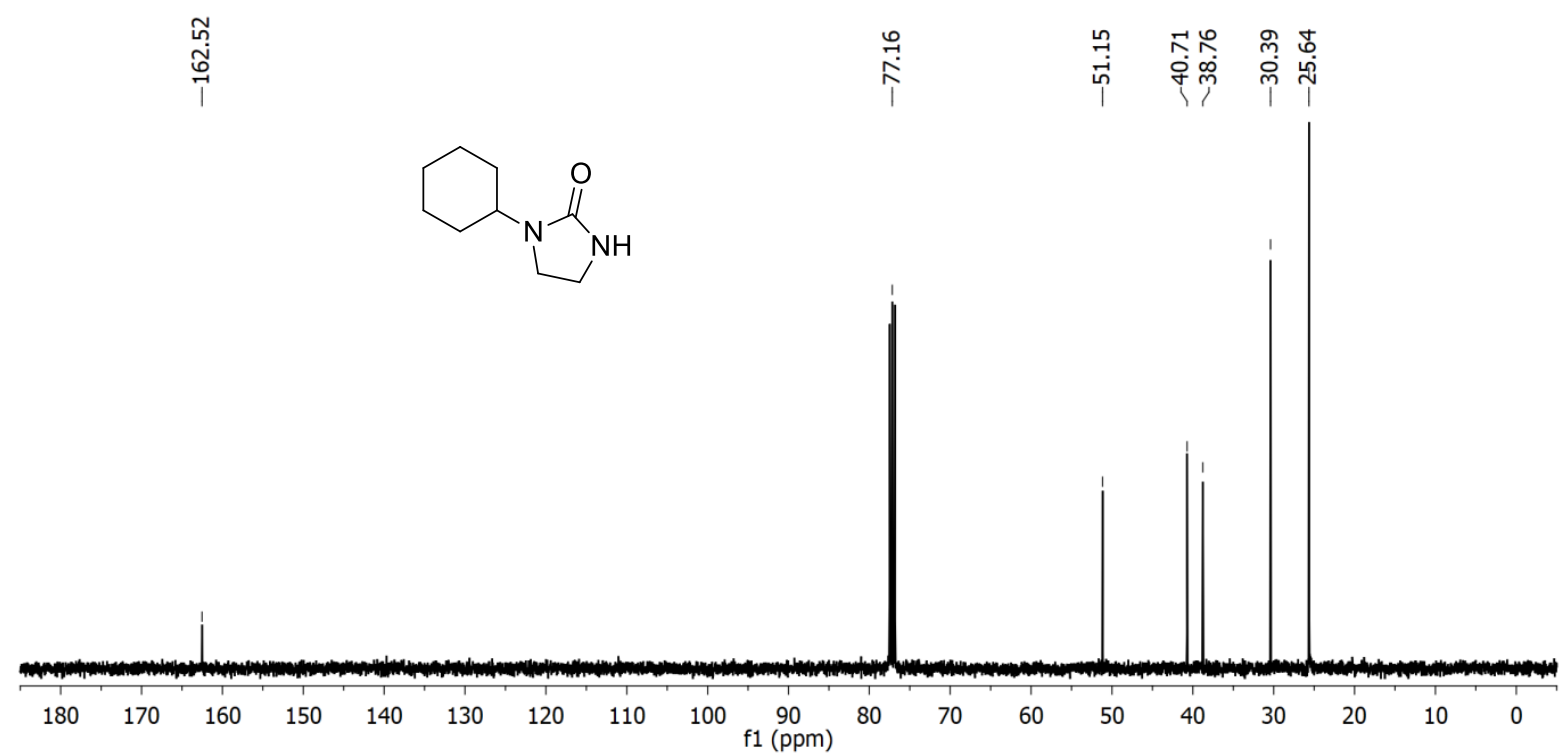

Figure S12. ${ }^{13} \mathrm{C}$ NMR spectrum (100 MHz, CDCl 3 , $298 \mathrm{~K}$ ) of 1-cyclohexylimidazolidin-2-one (L3H). 


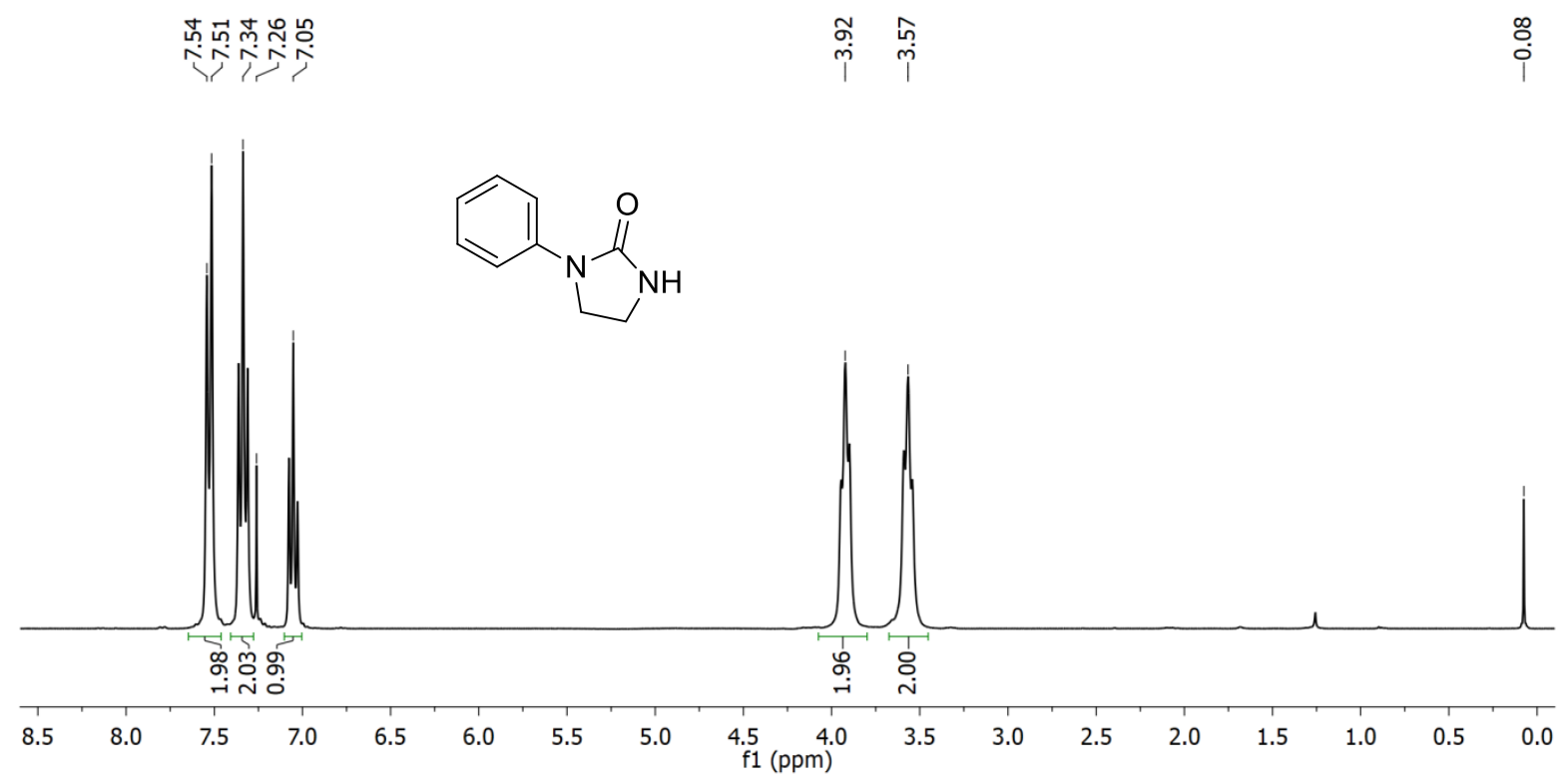

Figure S13. ${ }^{1} \mathrm{H}$ NMR spectrum ( $300 \mathrm{MHz}, \mathrm{CDCl}_{3}, 298 \mathrm{~K}$ ) of 1-phenylimidazolidin-2-one (L4H).

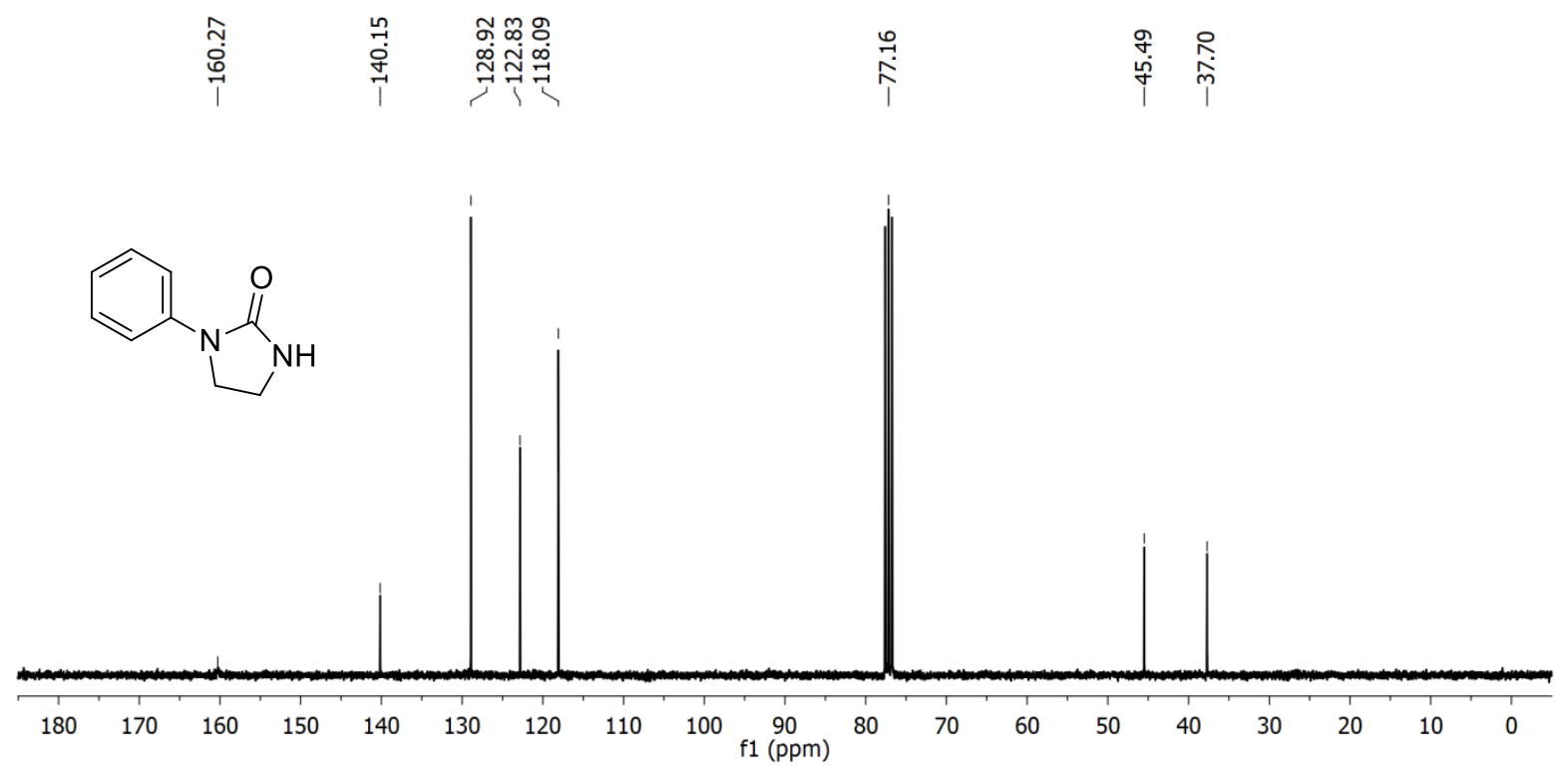

Figure S14. ${ }^{13} \mathrm{C}$ NMR spectrum (75 MHz, $\mathrm{CDCl}_{3}, 298 \mathrm{~K}$ ) of 1-phenylimidazolidin-2-one (L4H). 


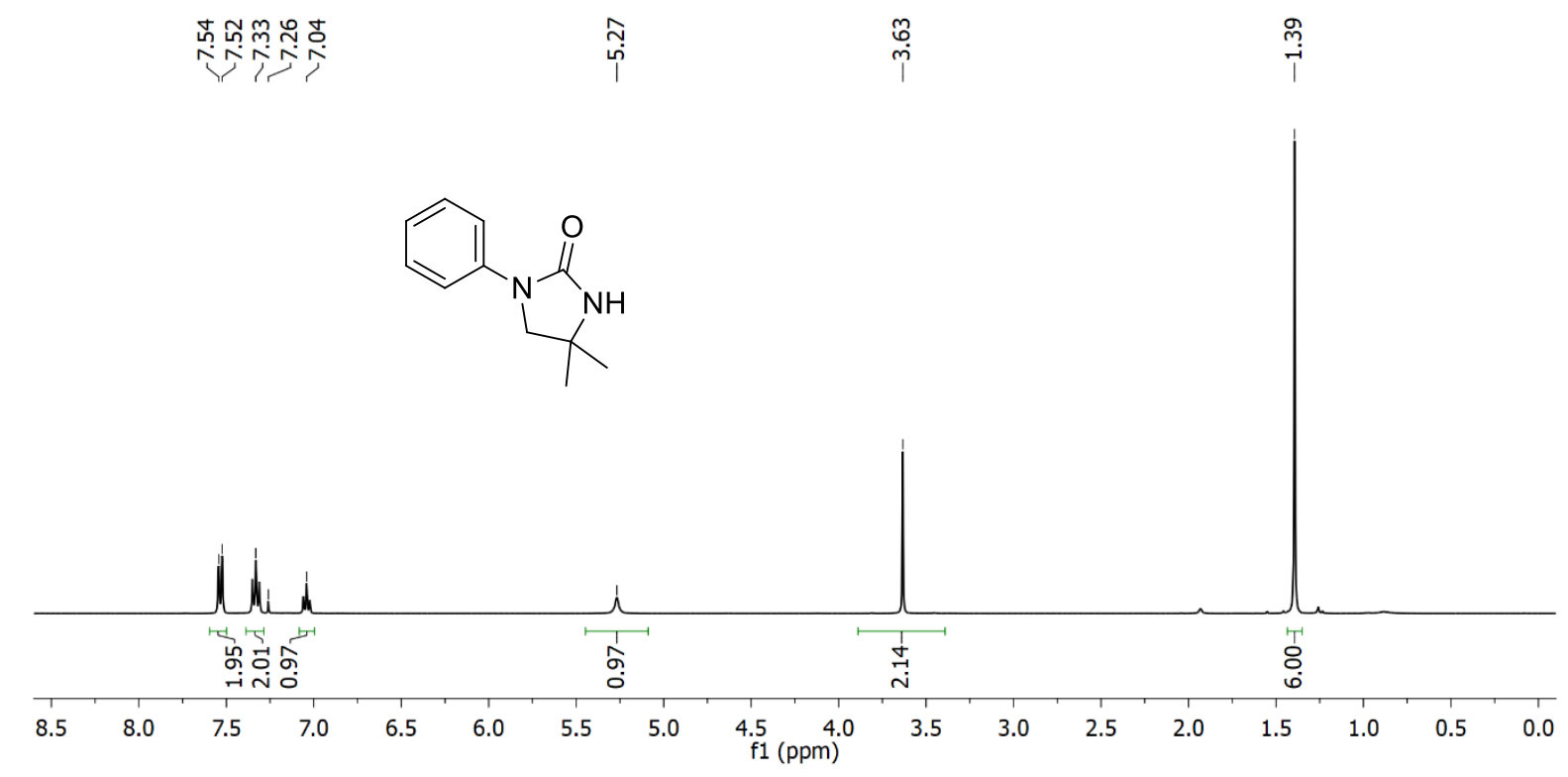

Figure S15. ${ }^{1} \mathrm{H}$ NMR spectrum $\left(400 \mathrm{MHz}, \mathrm{CDCl}_{3}, 298 \mathrm{~K}\right)$ of 4,4-dimethyl-1-phenylimidazolidin-2-one (L5H).

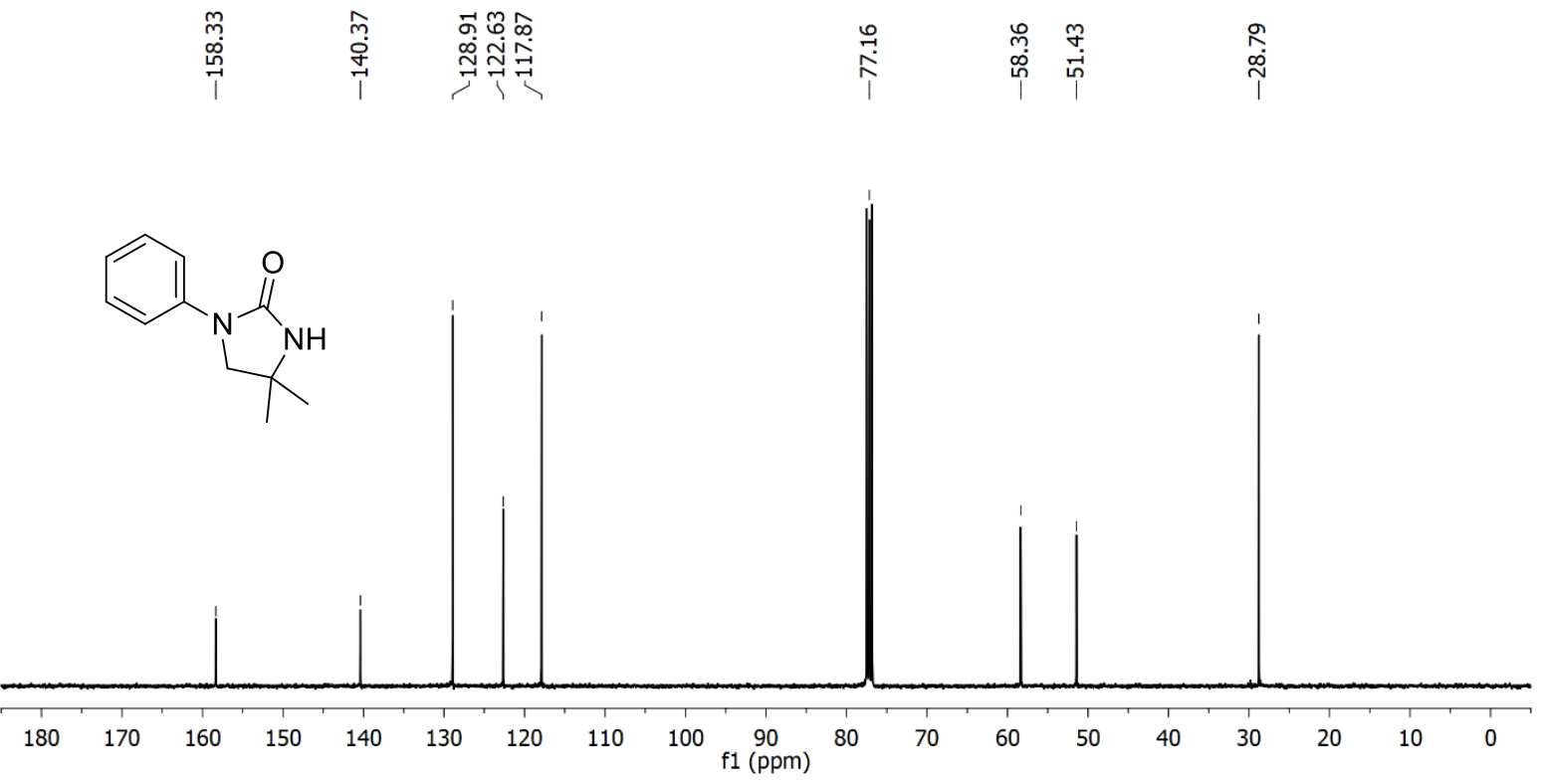

Figure S16. ${ }^{13} \mathrm{C}$ NMR spectrum $\left(75 \mathrm{MHz}, \mathrm{CDCl}_{3}, 298 \mathrm{~K}\right)$ of 4,4-dimethyl-1-phenylimidazolidin-2-one (L5H). 


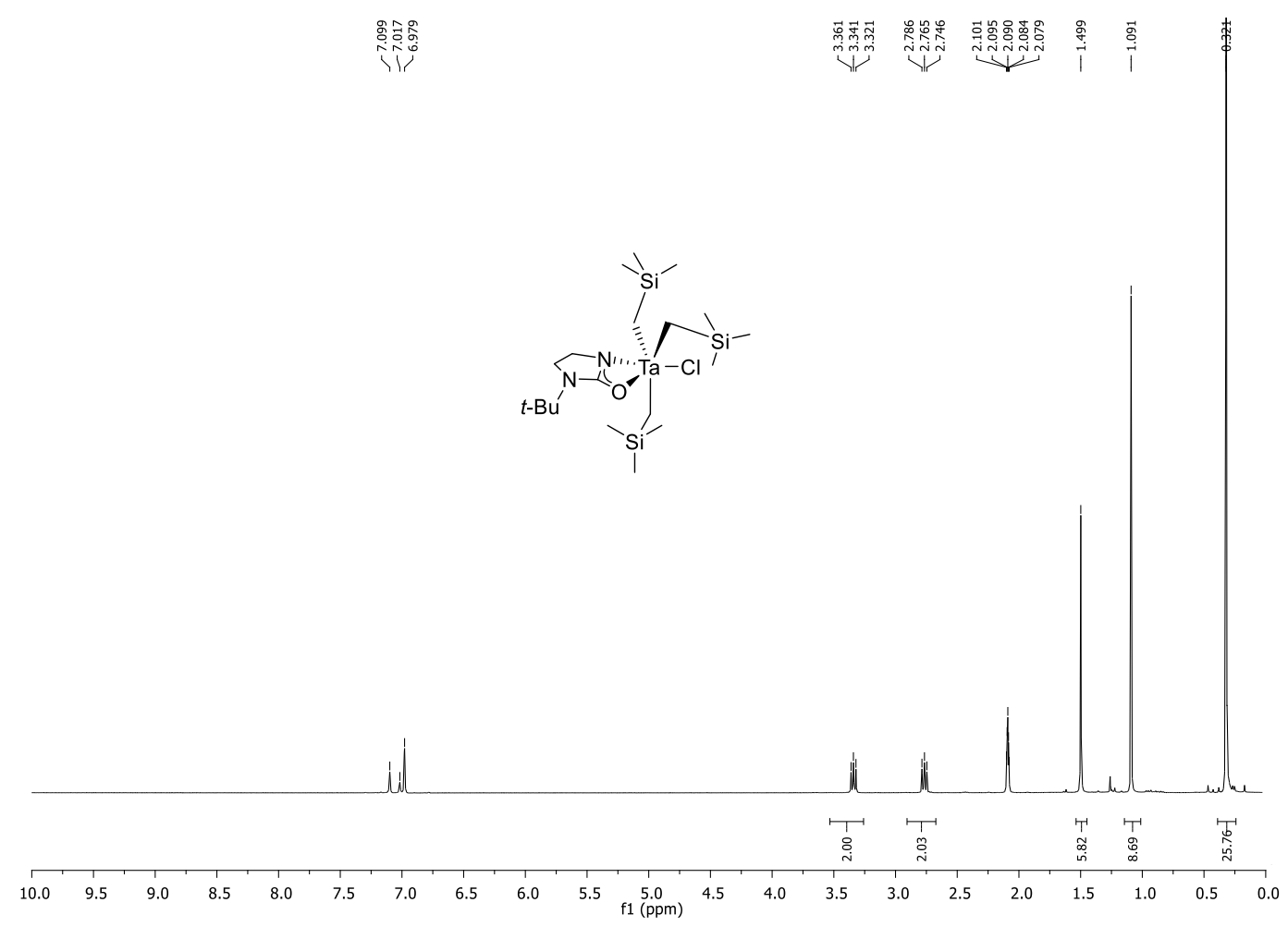

Figure S17. ${ }^{1} \mathrm{H}$ NMR spectrum (400 MHz, d $d_{8}$-toluene, $298 \mathrm{~K}$ ) of $\mathrm{L} 2 \mathrm{Ta}\left(\mathrm{CH}_{2} \mathrm{SiMe}_{3}\right)_{3} \mathrm{Cl}$.

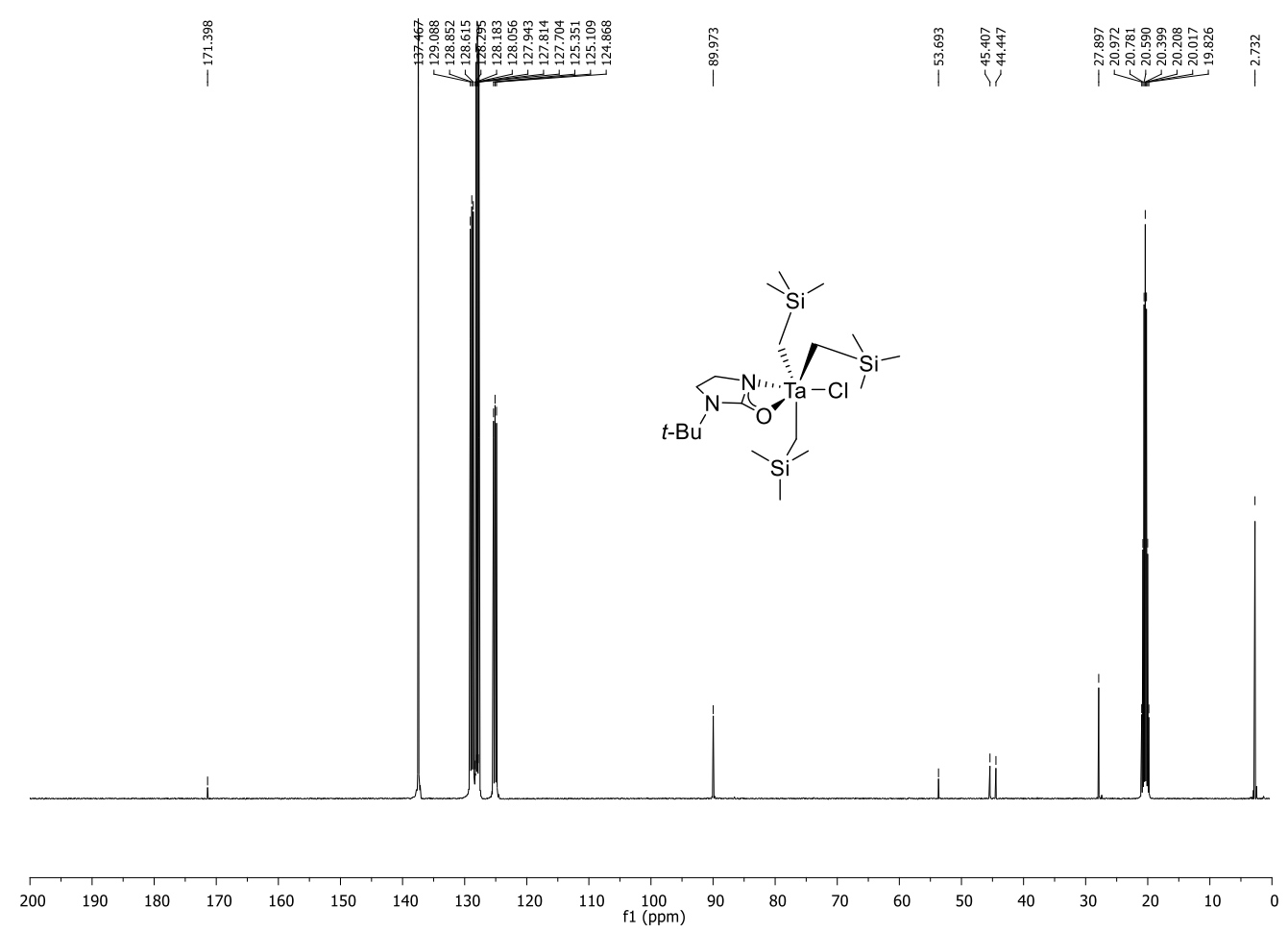

Figure S18. ${ }^{13} \mathrm{C}$ NMR spectrum ( $400 \mathrm{MHz}, \mathrm{d}_{8}$-toluene, $\left.298 \mathrm{~K}\right)$ of $\mathrm{L2Ta}\left(\mathrm{CH}_{2} \mathrm{SiMe}_{3}\right)_{3} \mathrm{Cl}$. 


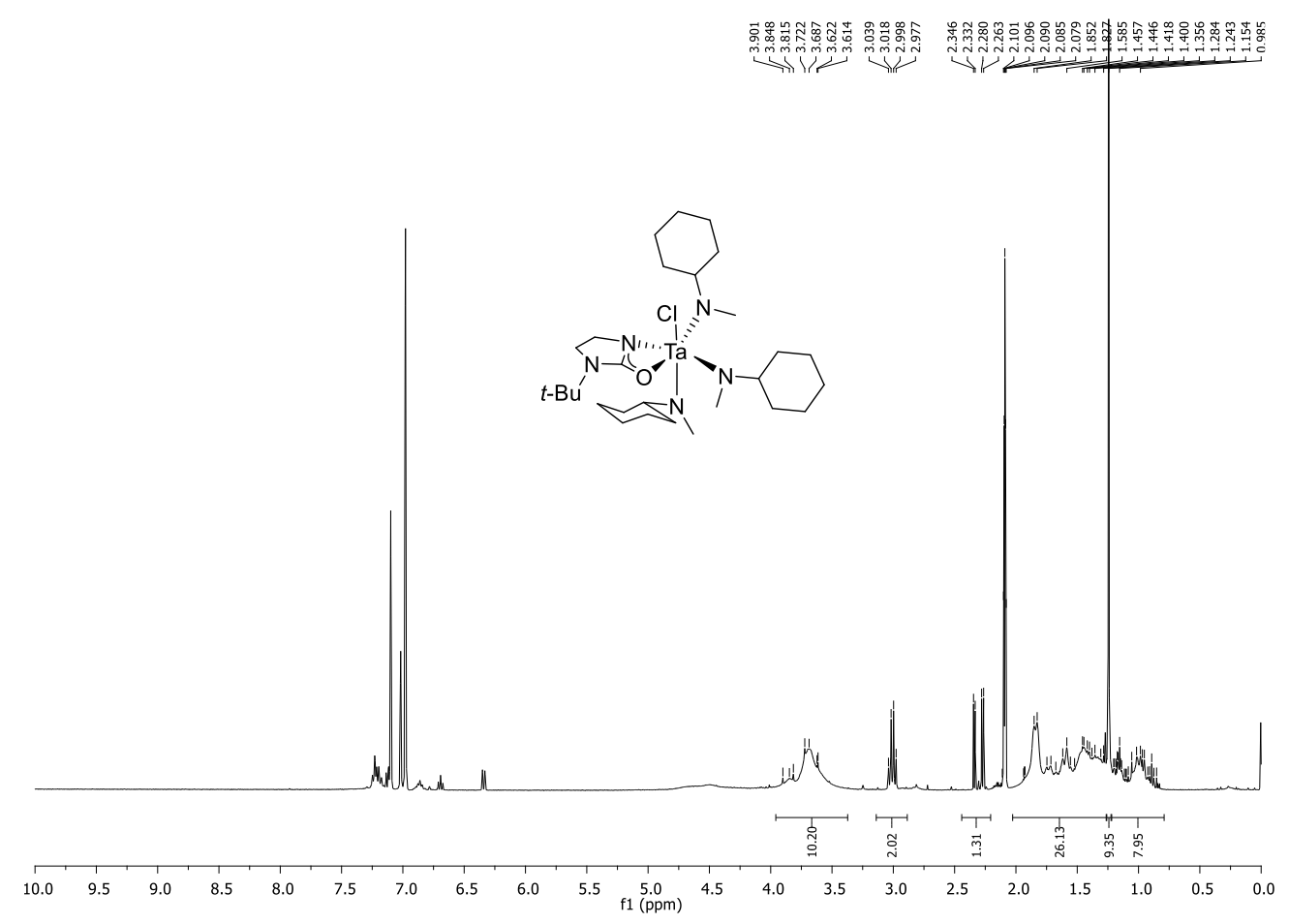

Figure S19. ${ }^{1} \mathrm{H}$ NMR spectrum $\left(400 \mathrm{MHz}, d_{8}\right.$-toluene, $\left.298 \mathrm{~K}\right)$ of $\mathrm{L} 2 \mathrm{Ta}(\mathrm{NMeCy})_{3} \mathrm{Cl}$.
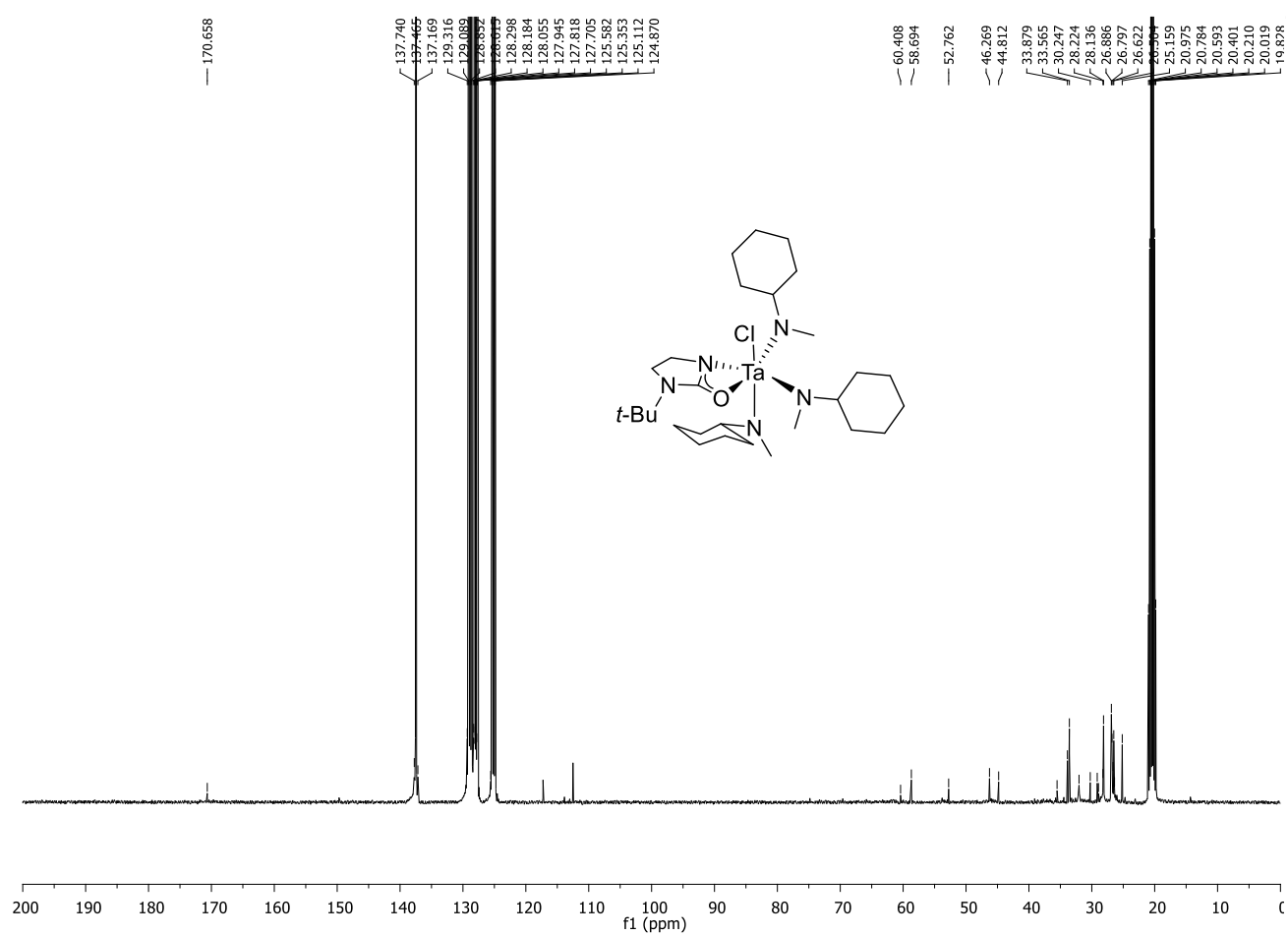

Figure S20. ${ }^{13} \mathrm{C}$ NMR spectrum $\left(400 \mathrm{MHz}, d_{8}\right.$-toluene, $\left.298 \mathrm{~K}\right)$ of $\mathrm{L} 2 \mathrm{Ta}(\mathrm{NMeCy})_{3} \mathrm{Cl}$. 


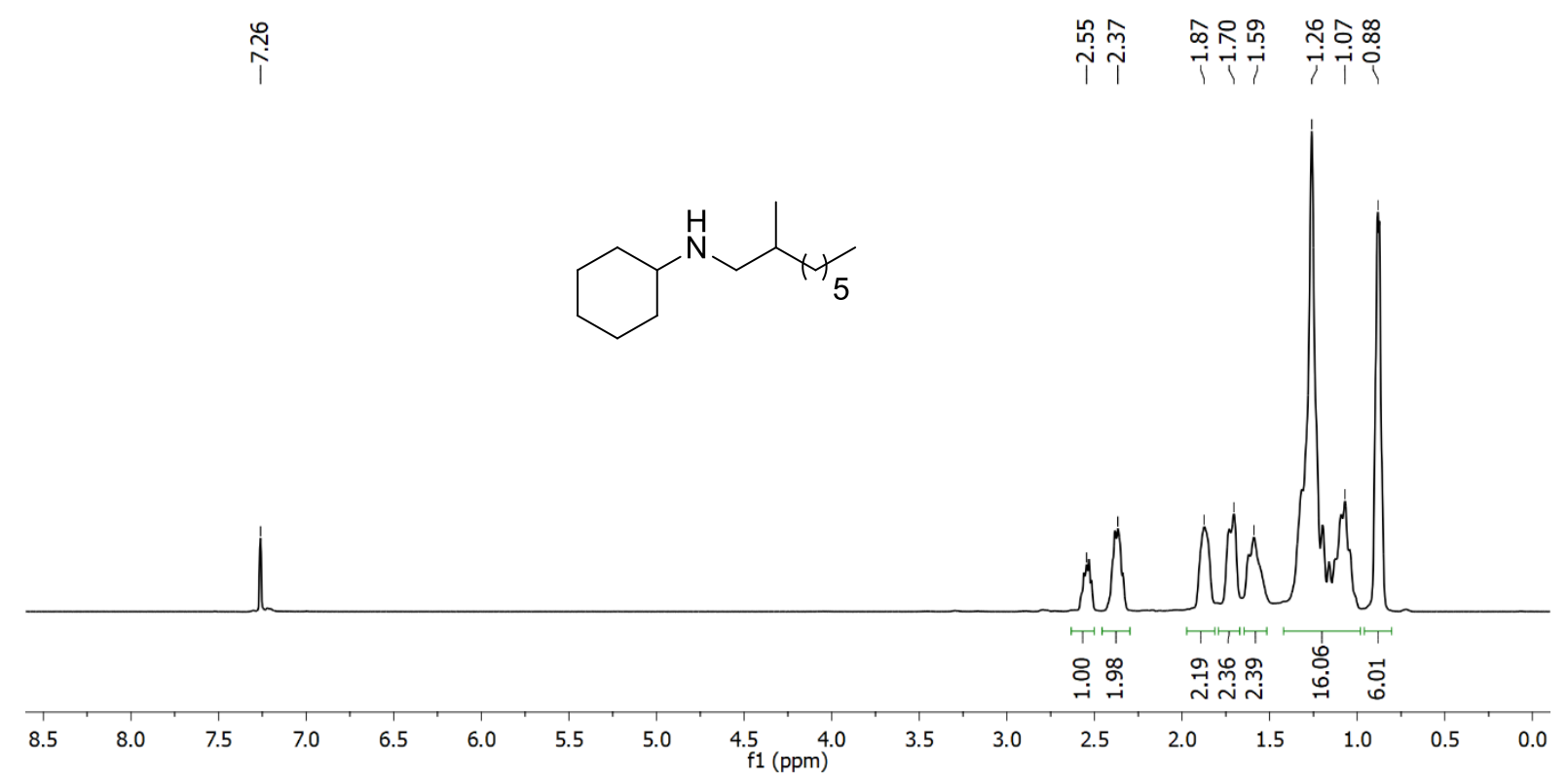

Figure S21. ${ }^{1} \mathrm{H}$ NMR spectrum (400 MHz, $\mathrm{CDCl}_{3}, 298 \mathrm{~K}$ ) of $\mathrm{N}$-(2-methyloctyl)cyclohexanamine (3).

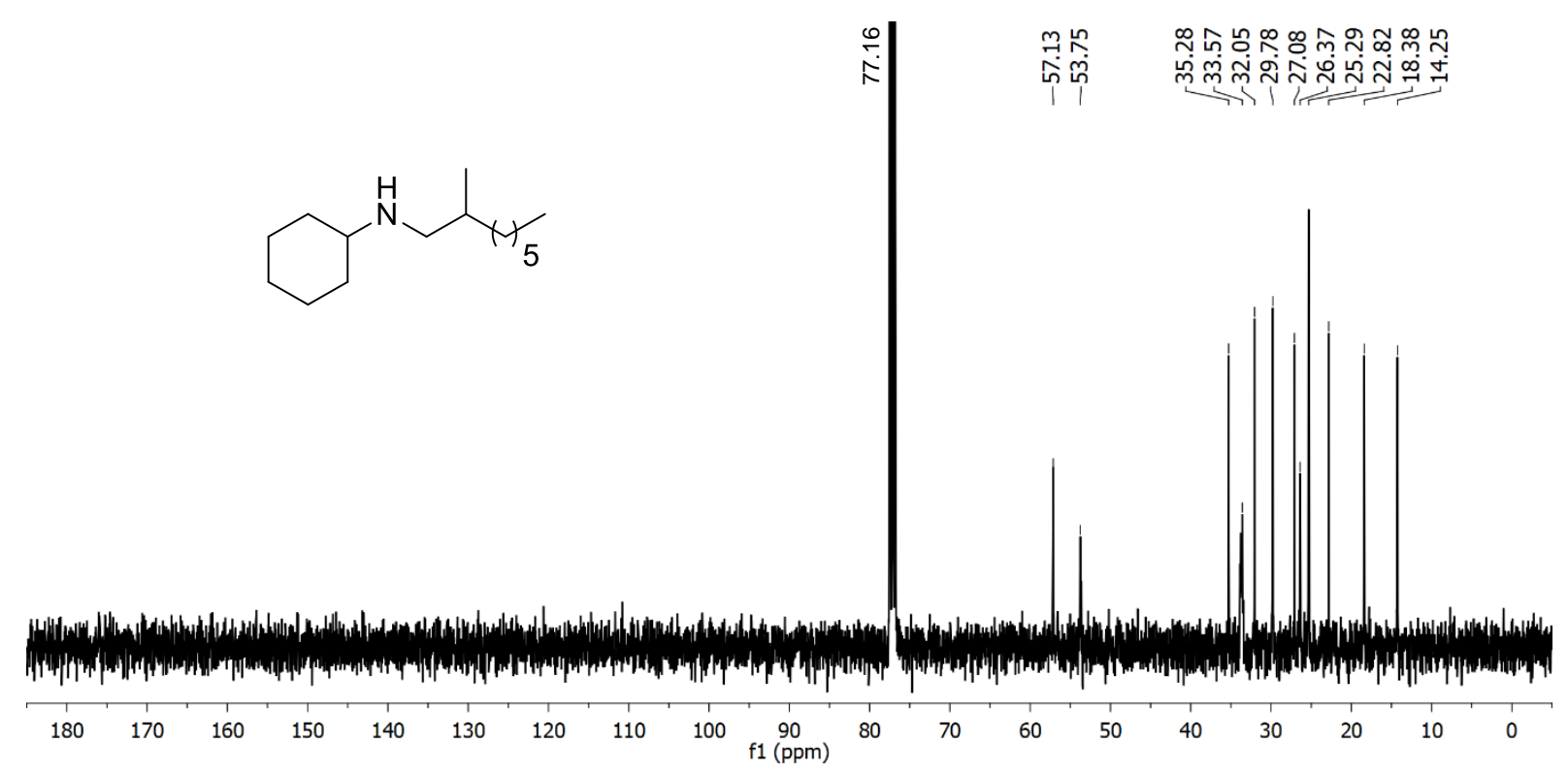

Figure S22. ${ }^{13} \mathrm{C}$ NMR spectrum $\left(100 \mathrm{MHz}, \mathrm{CDCl}_{3}, 298 \mathrm{~K}\right)$ of $\mathrm{N}$-(2-methyloctyl)cyclohexanamine (3). 


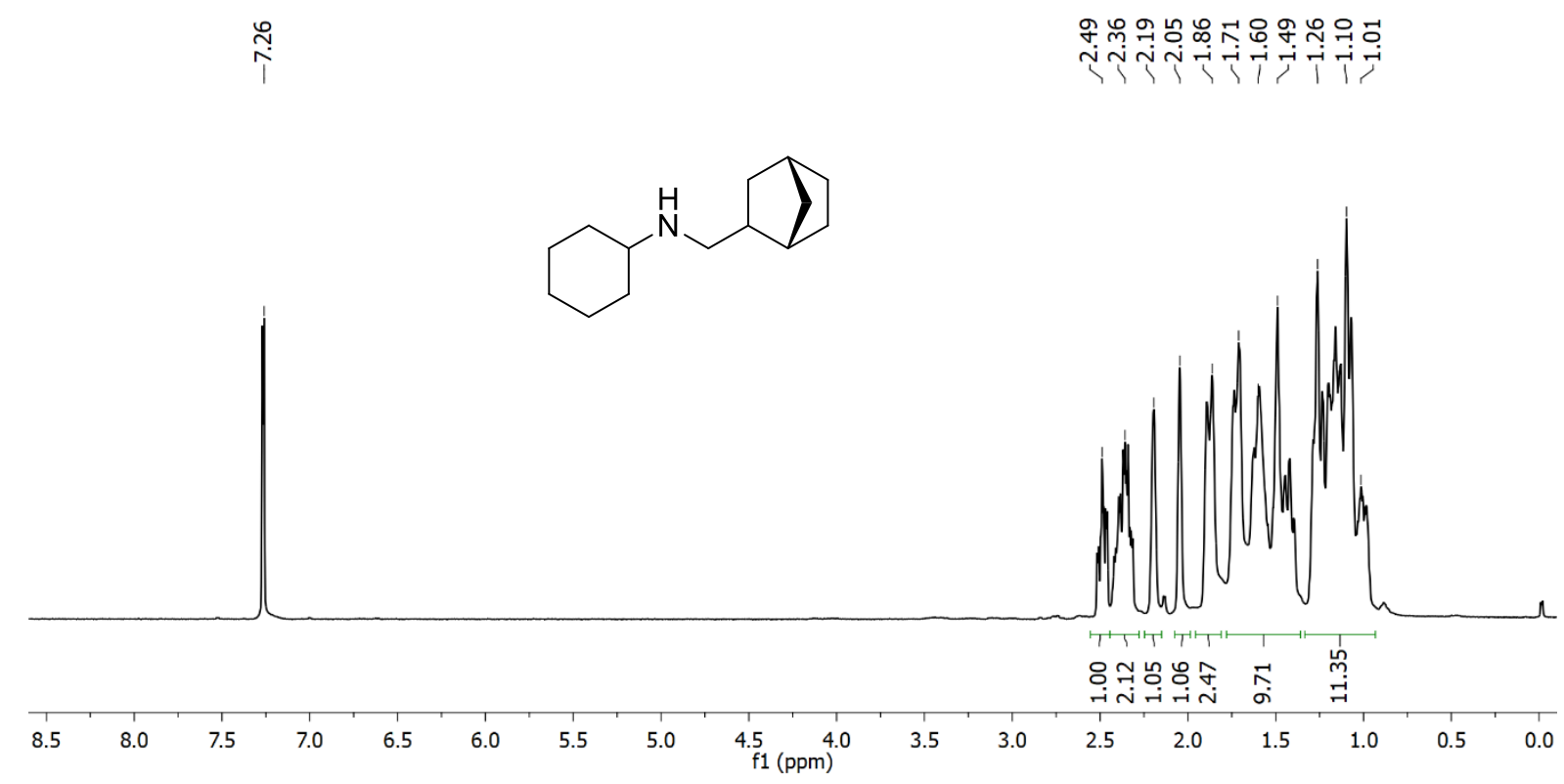

Figure S23. ${ }^{1} \mathrm{H}$ NMR spectrum (400 MHz, $\mathrm{CDCl}_{3}, 298 \mathrm{~K}$ ) of $\mathrm{N}$-(bicyclo[2.2.1] heptan-2-ylmethyl)cyclohexanamine (4).

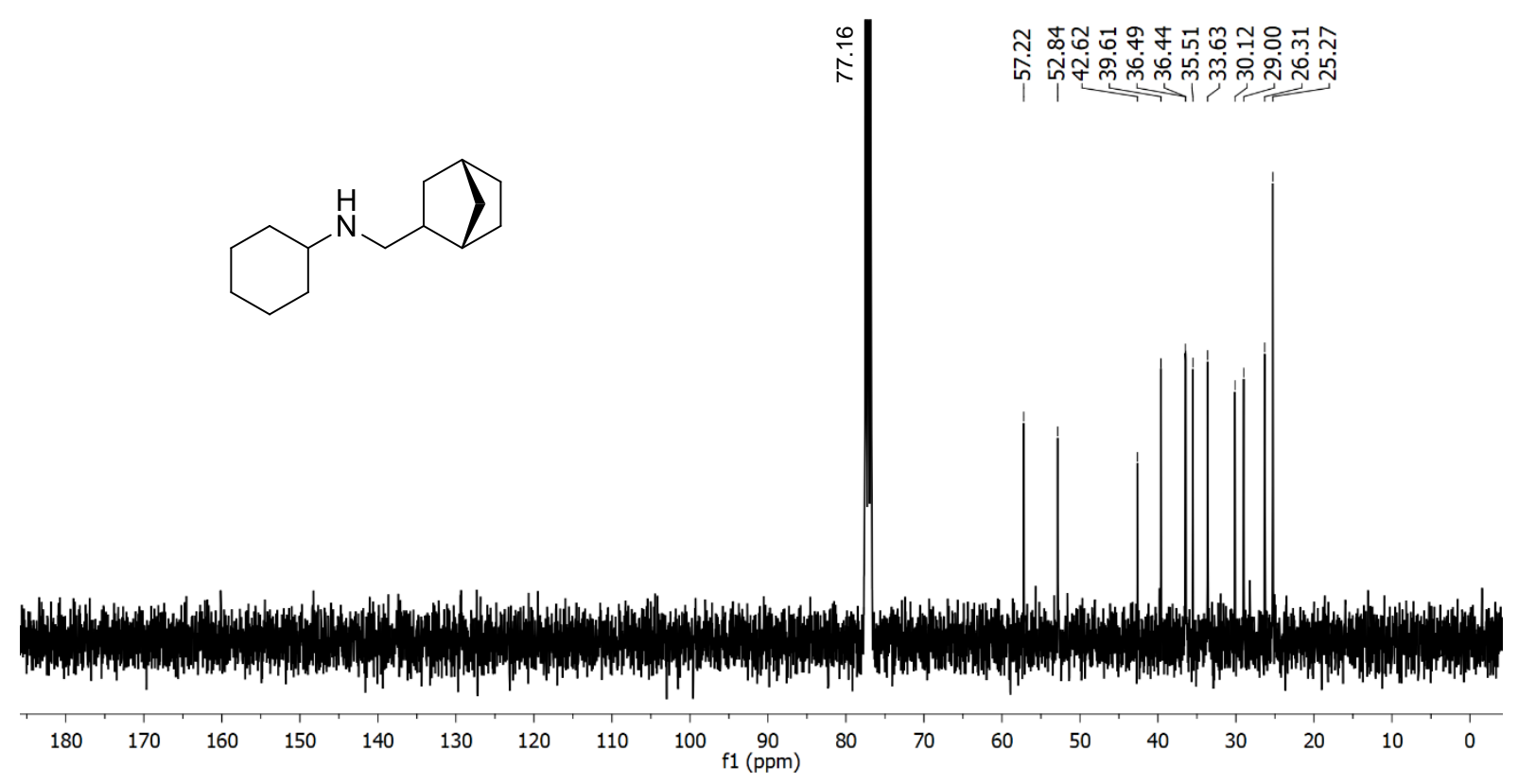

Figure S24. ${ }^{13} \mathrm{C}$ NMR spectrum (100 MHz, $\mathrm{CDCl}_{3}, 298 \mathrm{~K}$ ) of $\mathrm{N}$-(bicyclo[2.2.1] heptan-2-ylmethyl)cyclohexanamine (4). 


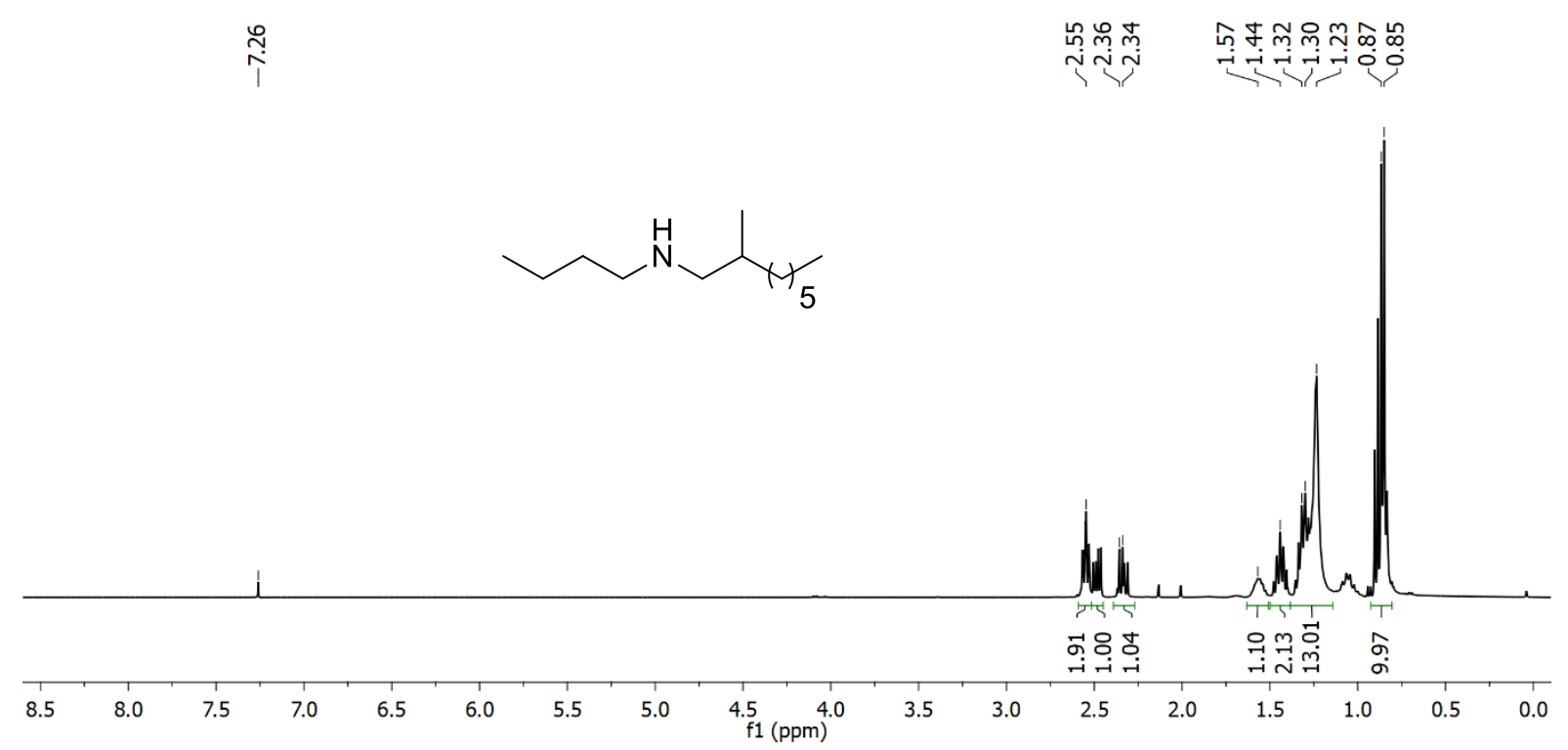

Figure S25. ${ }^{1} \mathrm{H}$ NMR spectrum $\left(400 \mathrm{MHz}, \mathrm{CDCl}_{3}, 298 \mathrm{~K}\right)$ of $\mathbf{N}$-butyl-2-methyloctan-1-amine (5).

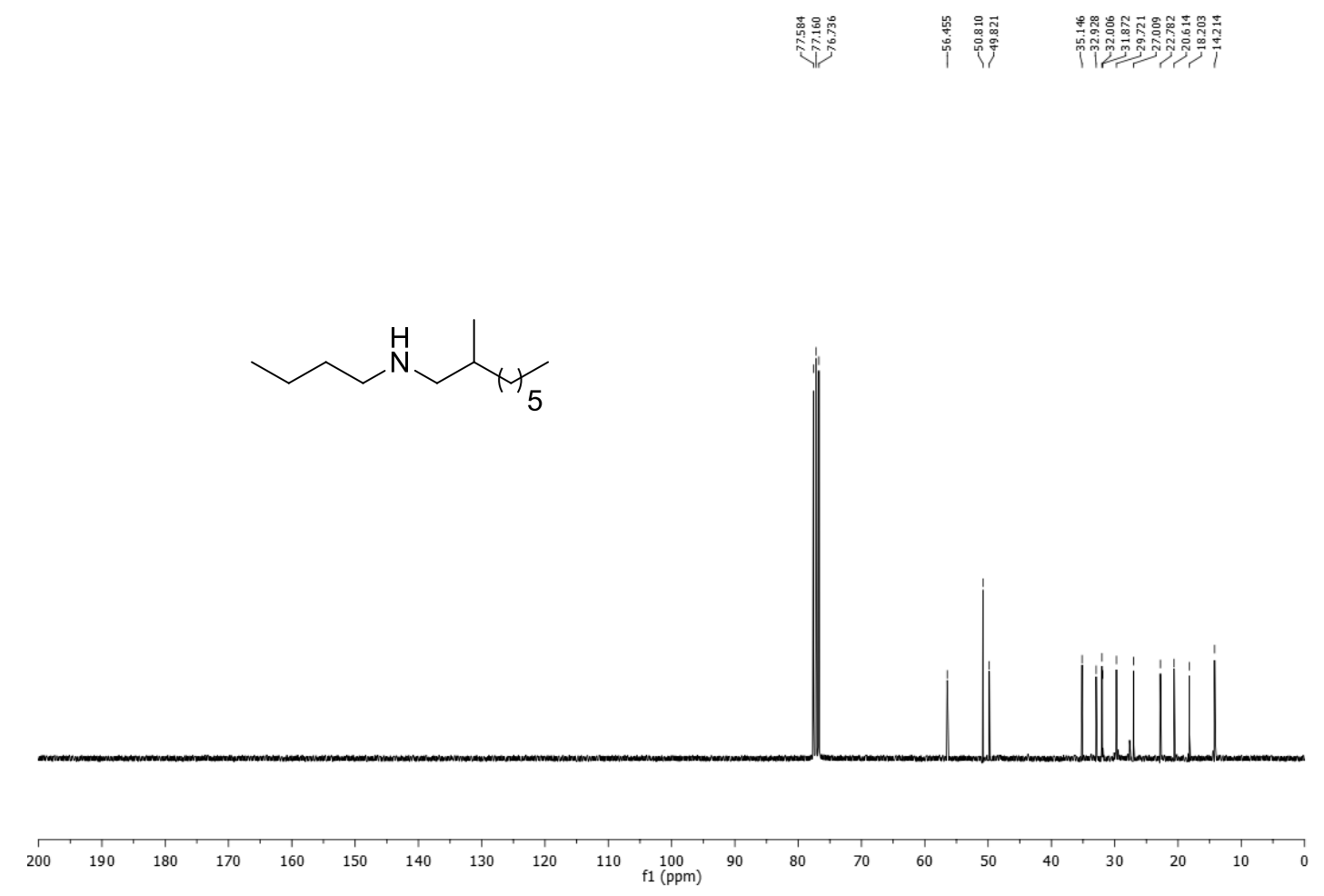

Figure S26. ${ }^{13} \mathrm{C}$ NMR spectrum $\left(100 \mathrm{MHz}, \mathrm{CDCl}_{3}, 298 \mathrm{~K}\right)$ of $\boldsymbol{N}$-butyl-2-methyloctan-1-amine (5). 


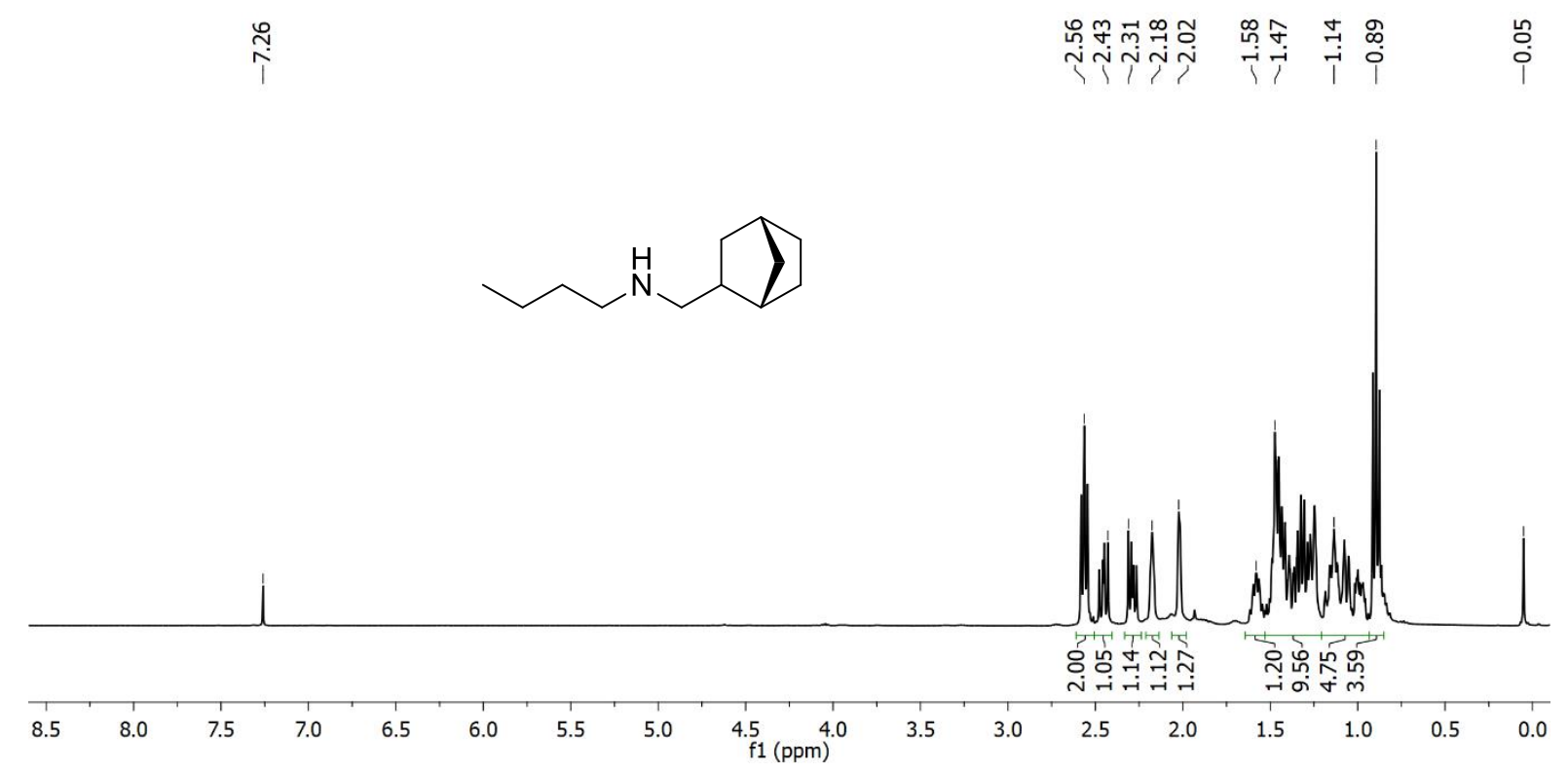

Figure S27. ${ }^{1} \mathrm{H}$ NMR spectrum $\left(400 \mathrm{MHz}, \mathrm{CDCl}_{3}, 298 \mathrm{~K}\right.$ ) of $\mathrm{N}$-bicyclo[2.2.1] heptan-2-ylmethyl)butan-1-amine (6).

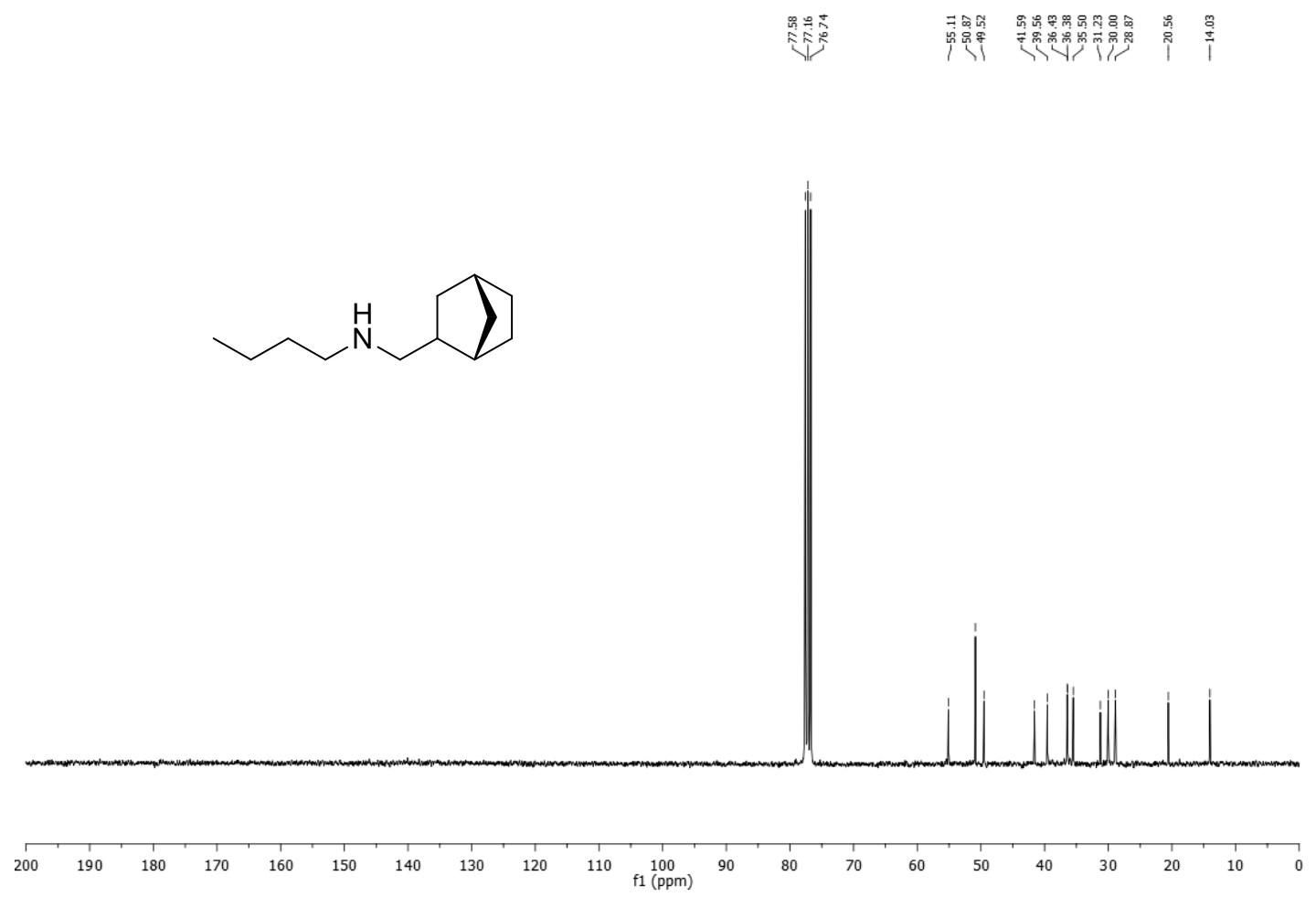

Figure S28. ${ }^{13} \mathrm{C}$ NMR spectrum (100 MHz, $\mathrm{CDCl}_{3}, 298 \mathrm{~K}$ ) of $\mathrm{N}$-bicyclo[2.2.1]heptan-2-ylmethyl)butan-1-amine (6). 


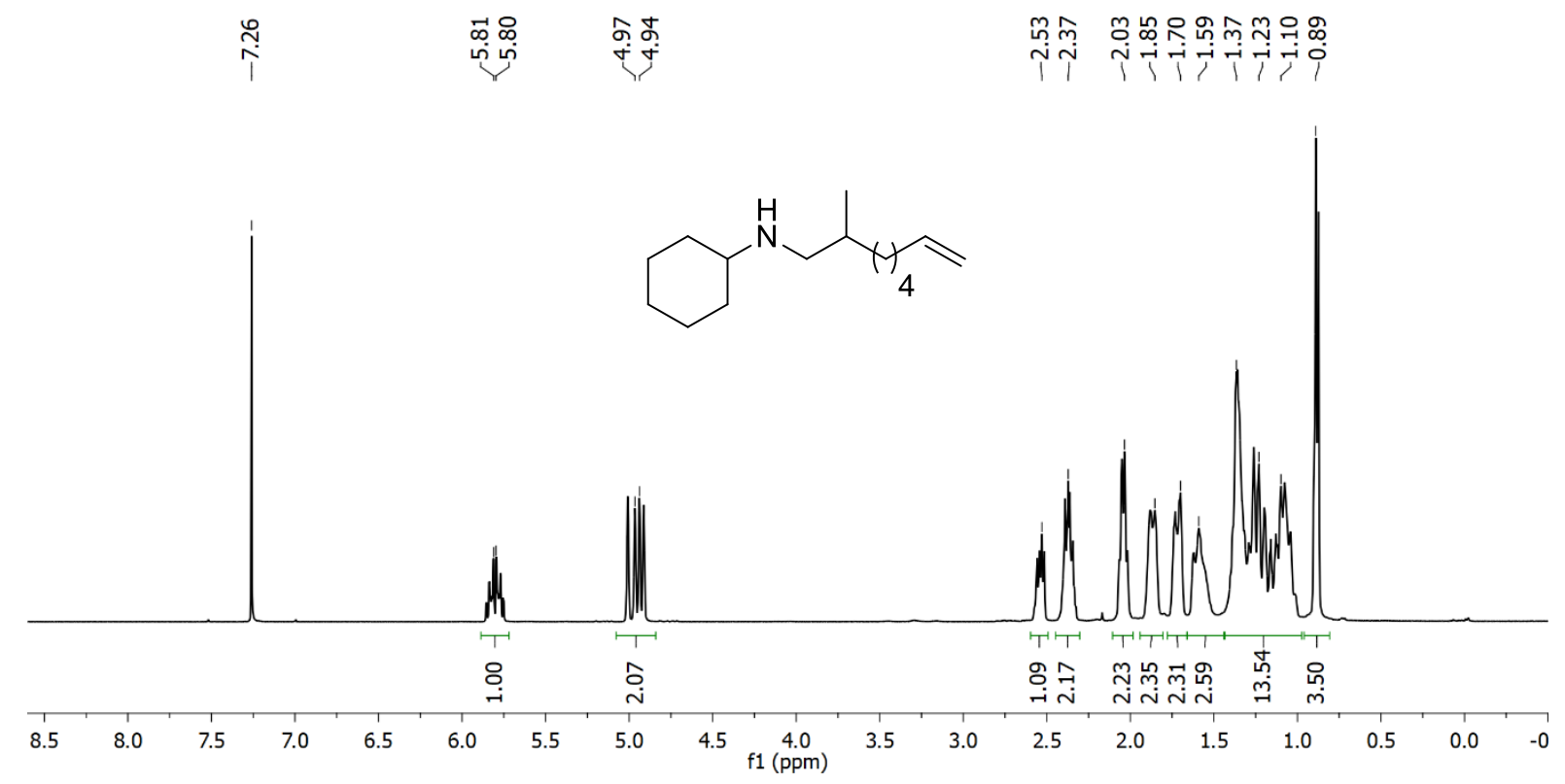

Figure S29. ${ }^{1} \mathrm{H}$ NMR spectrum $\left(400 \mathrm{MHz}, \mathrm{CDCl}_{3}, 298 \mathrm{~K}\right)$ of $\mathrm{N}$-(2-methyloct-7-en-1-yl)cyclohexanamine (7).

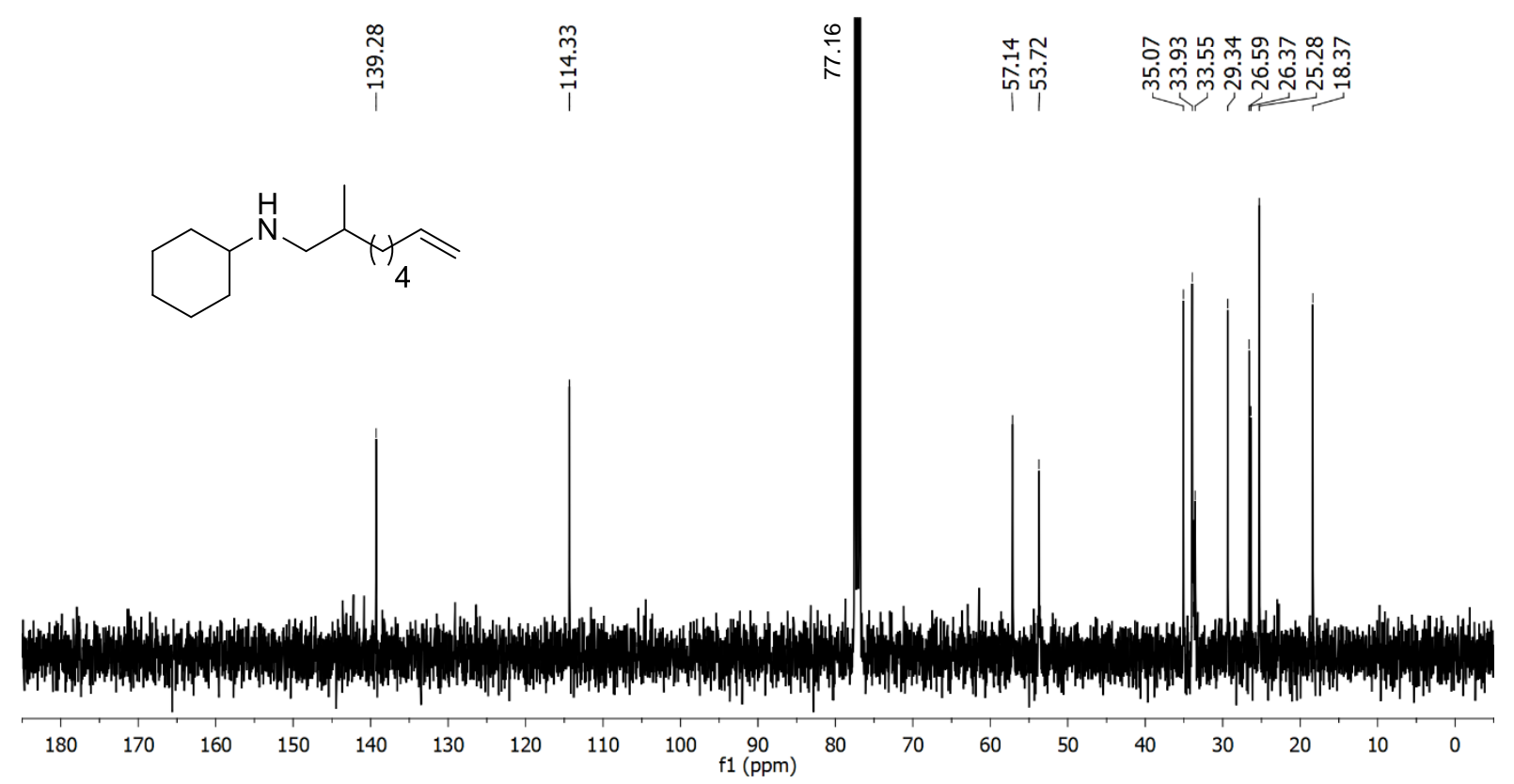

Figure S30. ${ }^{13} \mathrm{C}$ NMR spectrum (100 MHz, $\mathrm{CDCl}_{3}, 298 \mathrm{~K}$ ) of $\mathrm{N}$-(2-methyloct-7-en-1-yl)cyclohexanamine (7). 


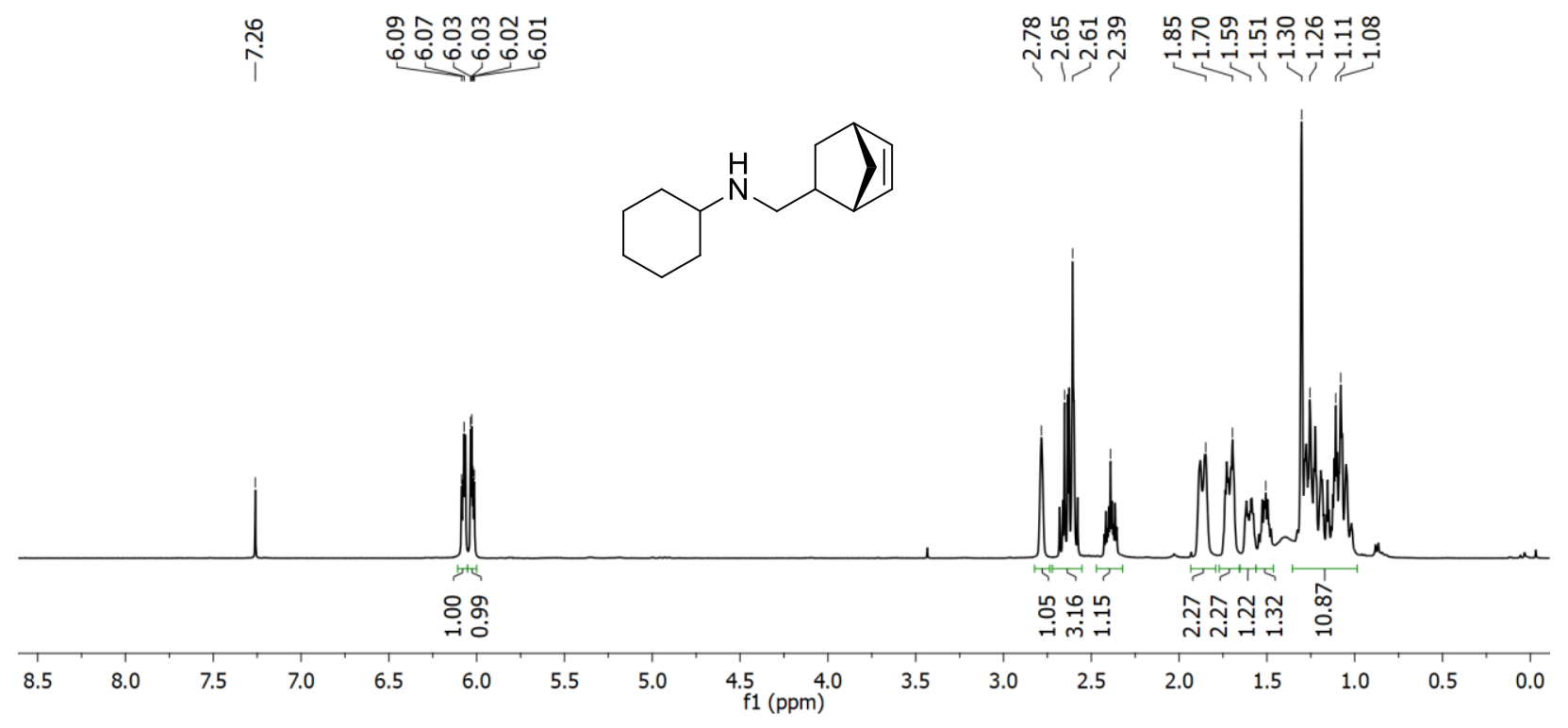

Figure S31. ${ }^{1} \mathrm{H}$ NMR spectrum (400 MHz, $\mathrm{CDCl}_{3}, 298 \mathrm{~K}$ ) of $\mathrm{N}$-bicyclo[2.2.1] hept-5-en-2-ylmethyl)cyclohexanamine (8).

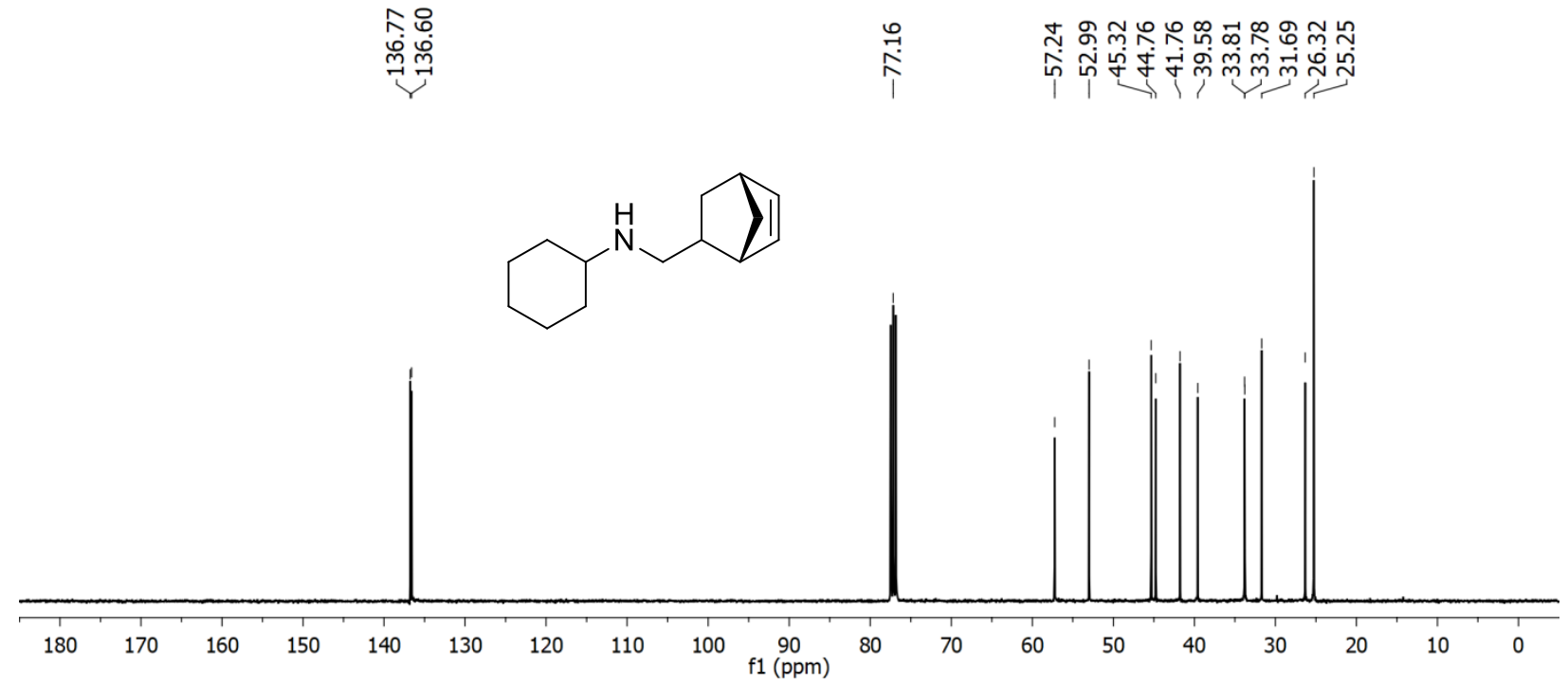

Figure S32. ${ }^{13} \mathrm{C}$ NMR spectrum (100 MHz, $\mathrm{CDCl}_{3}, 298 \mathrm{~K}$ ) of $\mathrm{N}$-bicyclo[2.2.1] hept-5-en-2-ylmethyl)cyclohexanamine (8). 


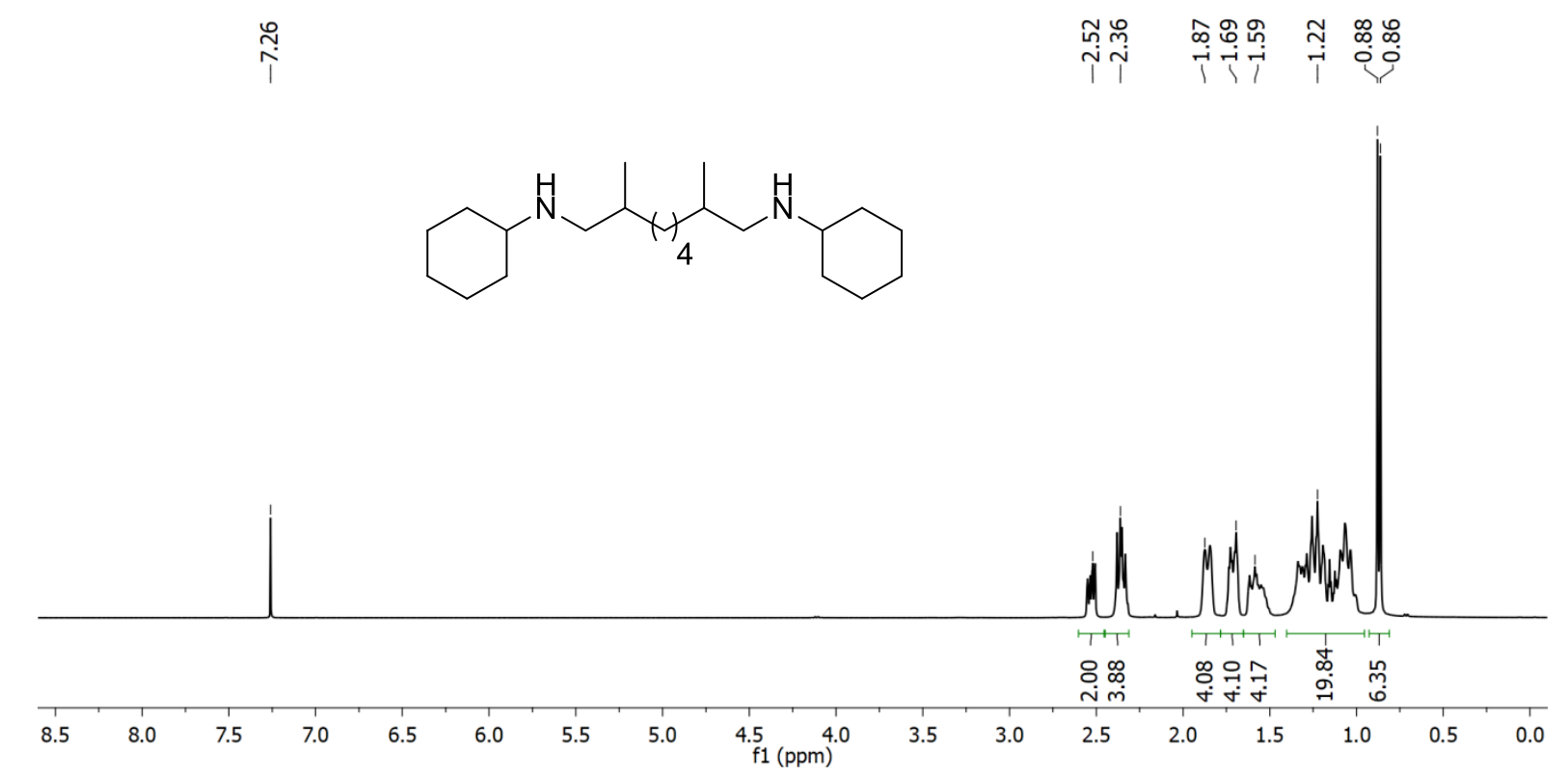

Figure S33. ${ }^{1} \mathrm{H}$ NMR spectrum $\left(400 \mathrm{MHz}, \mathrm{CDCl}_{3}, 298 \mathrm{~K}\right)$ of $N^{1}, N^{8}$-dicyclohexyl-2,7-dimethyloctane-1,8-diamine (9).

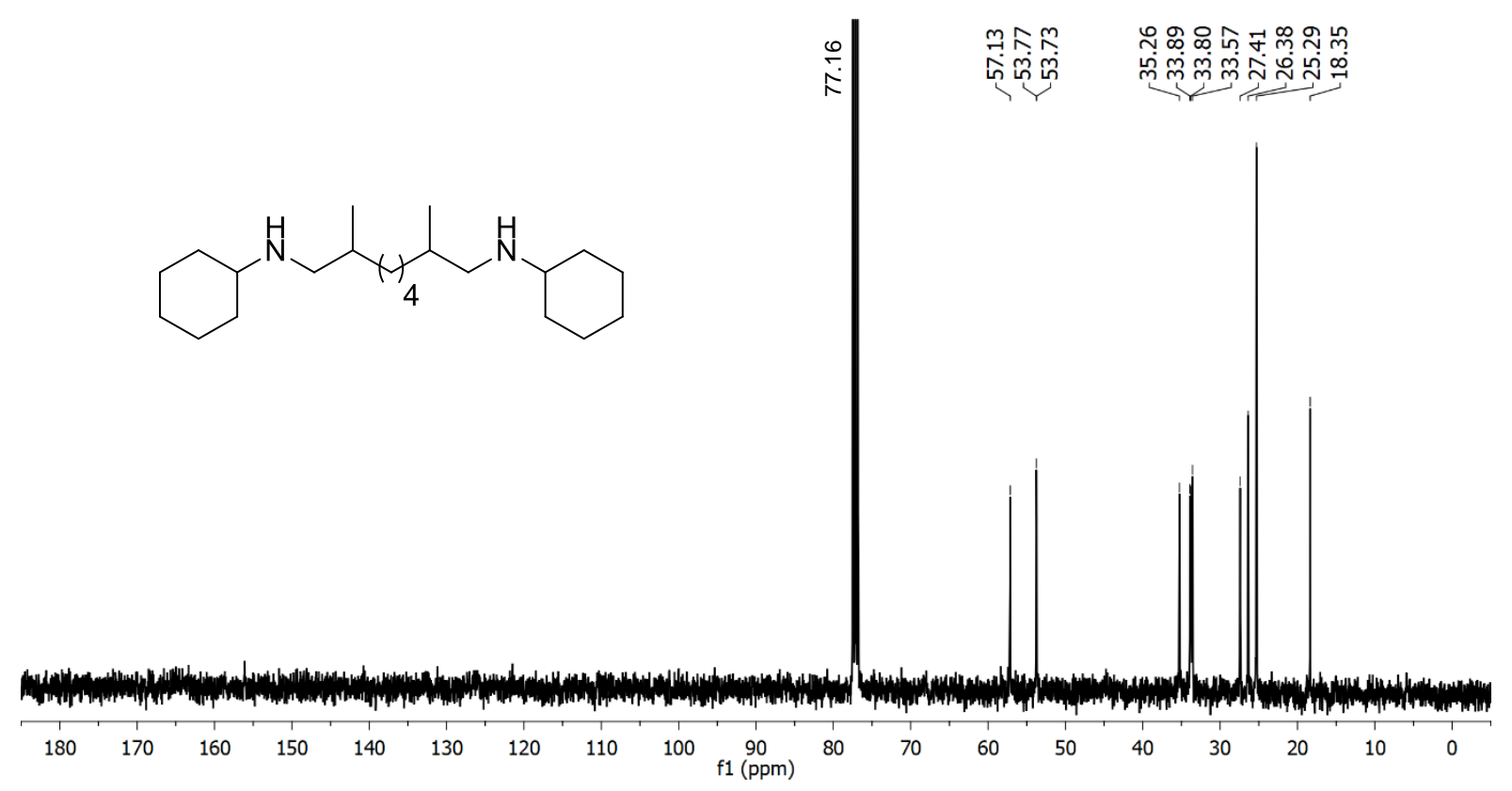

Figure S34. ${ }^{13} \mathrm{C}$ NMR spectrum $\left(100 \mathrm{MHz}, \mathrm{CDCl}_{3}, 298 \mathrm{~K}\right)$ of $\mathrm{N}^{1}, \mathrm{~N}^{8}$-dicyclohexyl-2,7-dimethyloctane-1,8-diamine (9). 


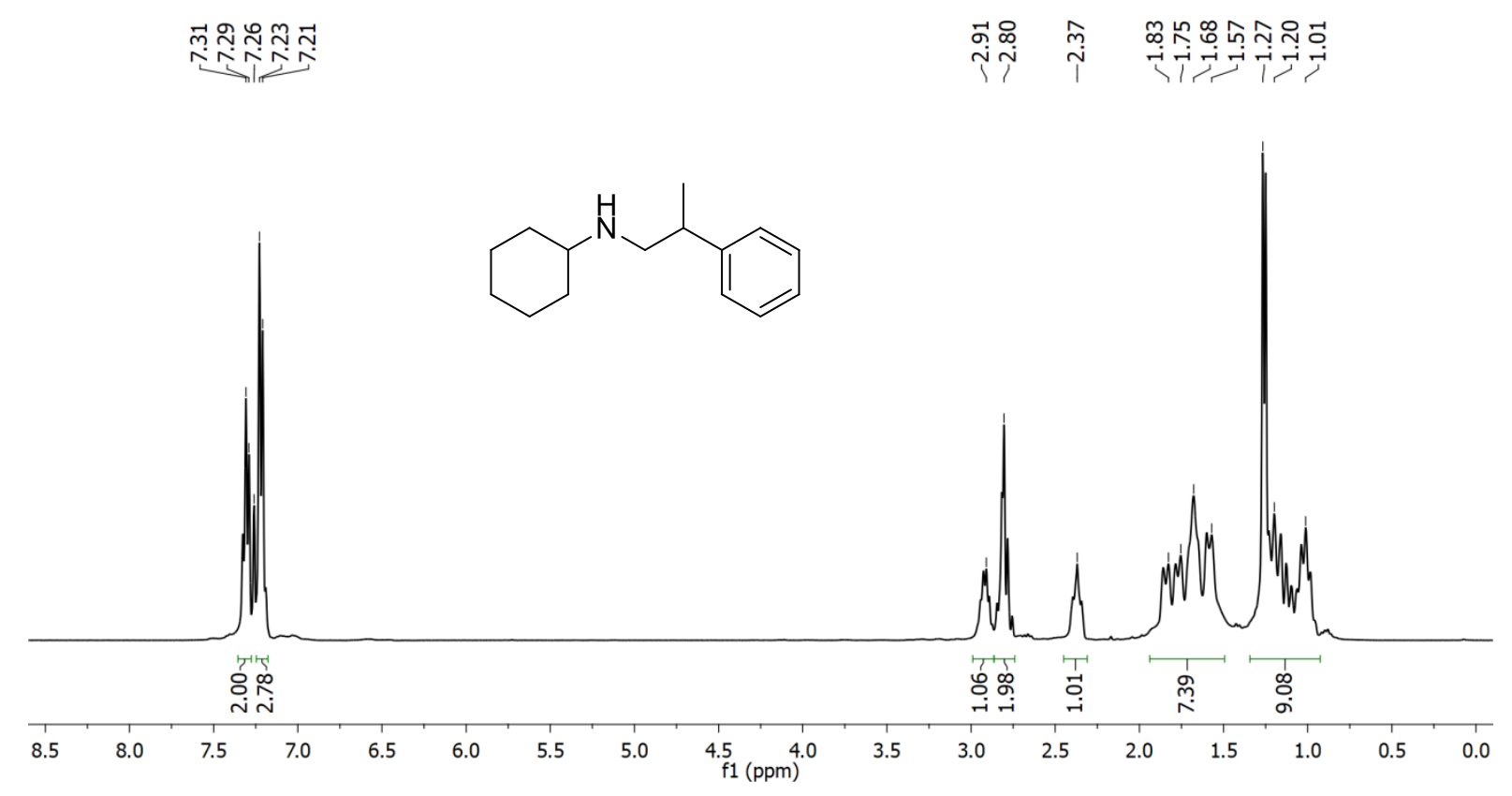

Figure S35. ${ }^{1} \mathrm{H}$ NMR spectrum $\left(400 \mathrm{MHz}, \mathrm{CDCl}_{3}, 298 \mathrm{~K}\right)$ of $\mathrm{N}$-(2-phenylpropyl)cyclohexanamine (10).

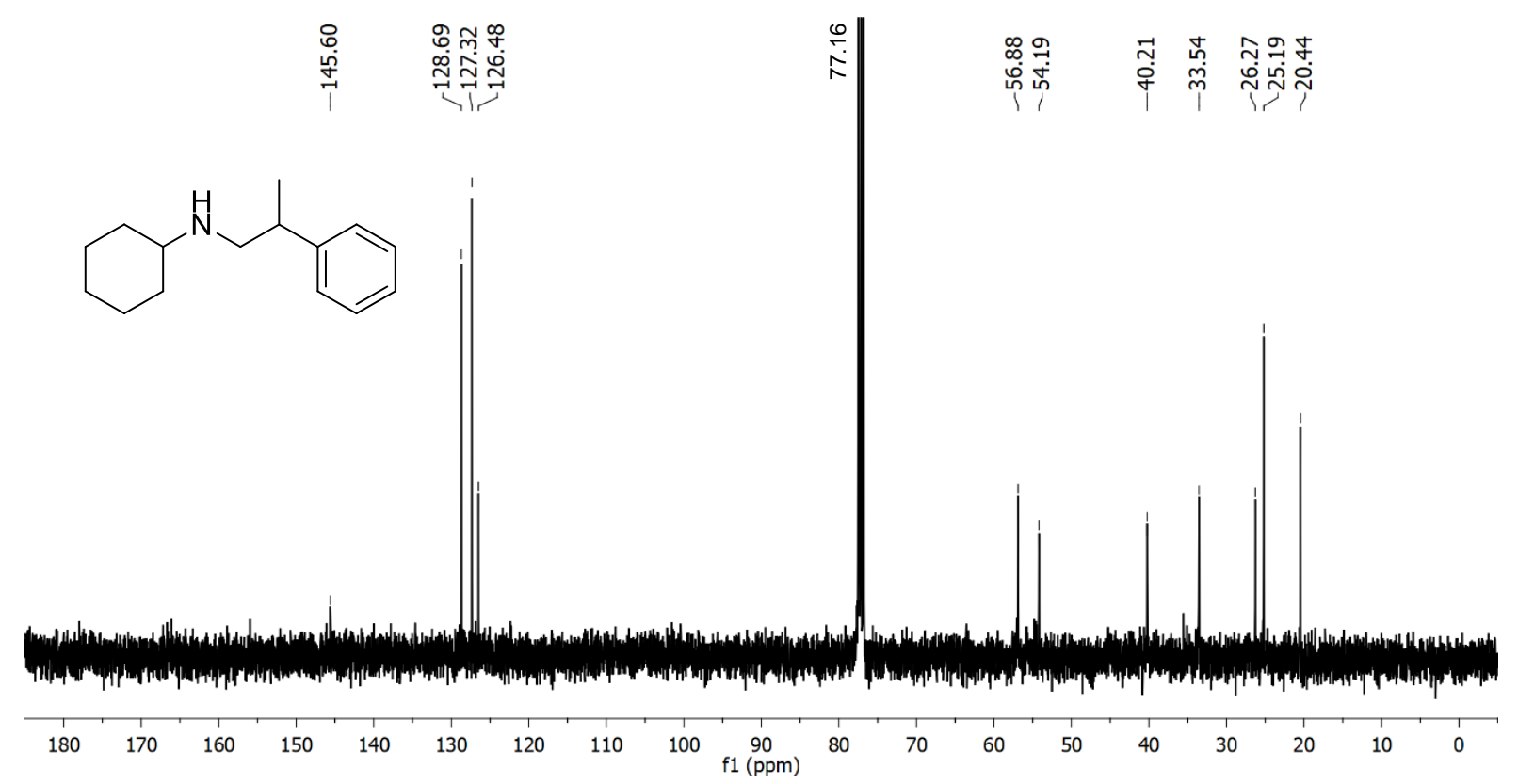

Figure S36. ${ }^{13} \mathrm{C}$ NMR spectrum (100 MHz, $\mathrm{CDCl}_{3}, 298 \mathrm{~K}$ ) of $\mathrm{N}$-(2-phenylpropyl)cyclohexanamine (10). 


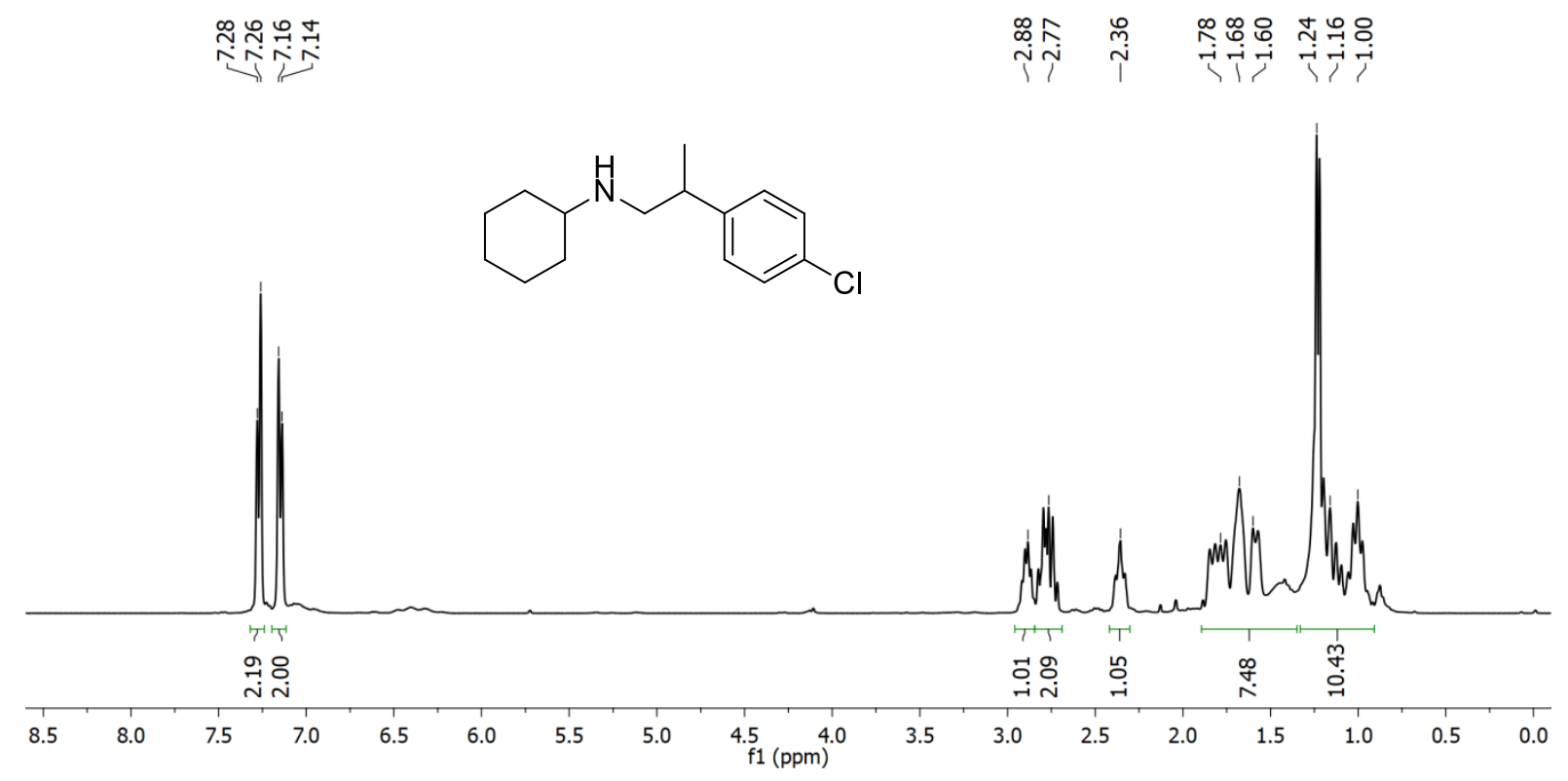

Figure S37. ${ }^{1} \mathrm{H}$ NMR spectrum $\left(400 \mathrm{MHz}, \mathrm{CDCl}_{3}, 298 \mathrm{~K}\right)$ of $\mathrm{N}$-(2-(4-chlorophenyl)propyl)cyclohexanamine (11).

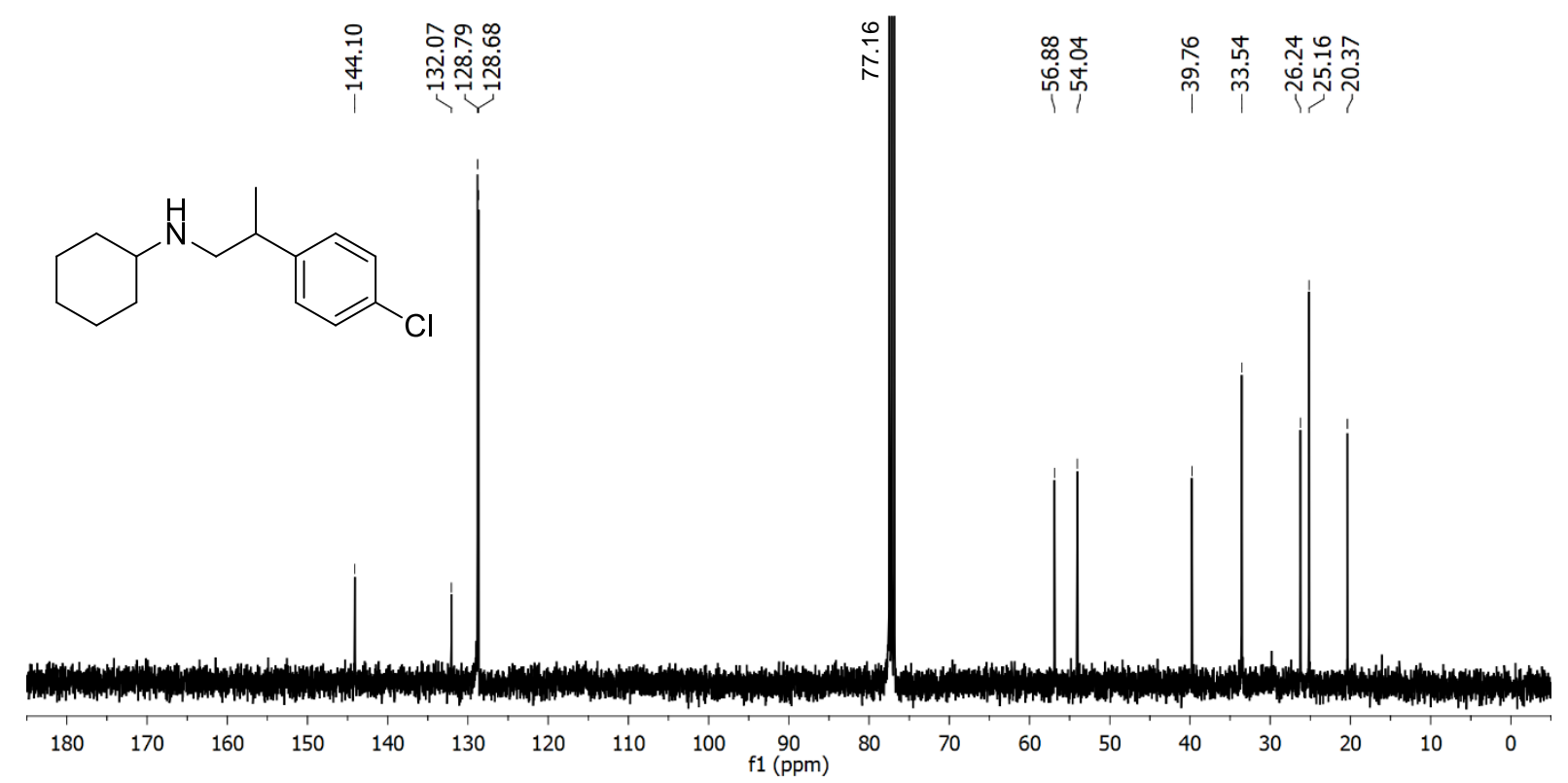

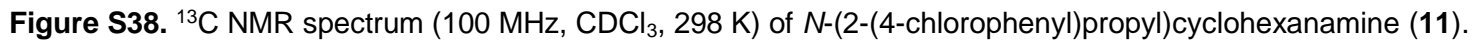


<smiles>C[Si](C)(C)CCCNC1CCCCC1</smiles>

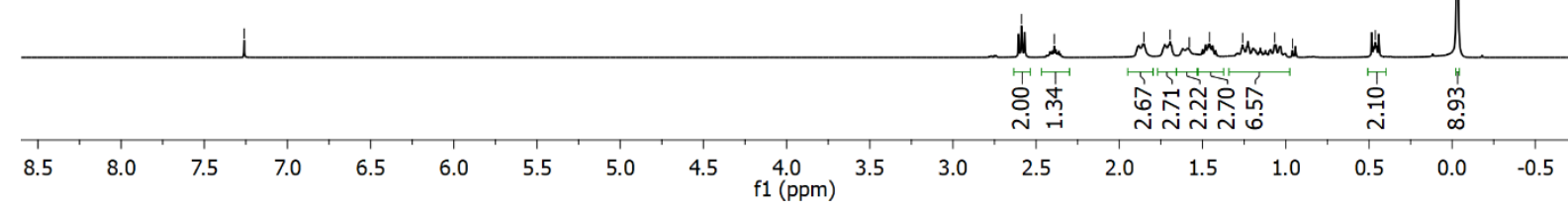

Figure S39. ${ }^{1} \mathrm{H}$ NMR spectrum $\left(400 \mathrm{MHz}, \mathrm{CDCl}_{3}, 298 \mathrm{~K}\right)$ of $\mathrm{N}$-(3-(trimethylsilyl)propyl)cyclohexanamine (12).

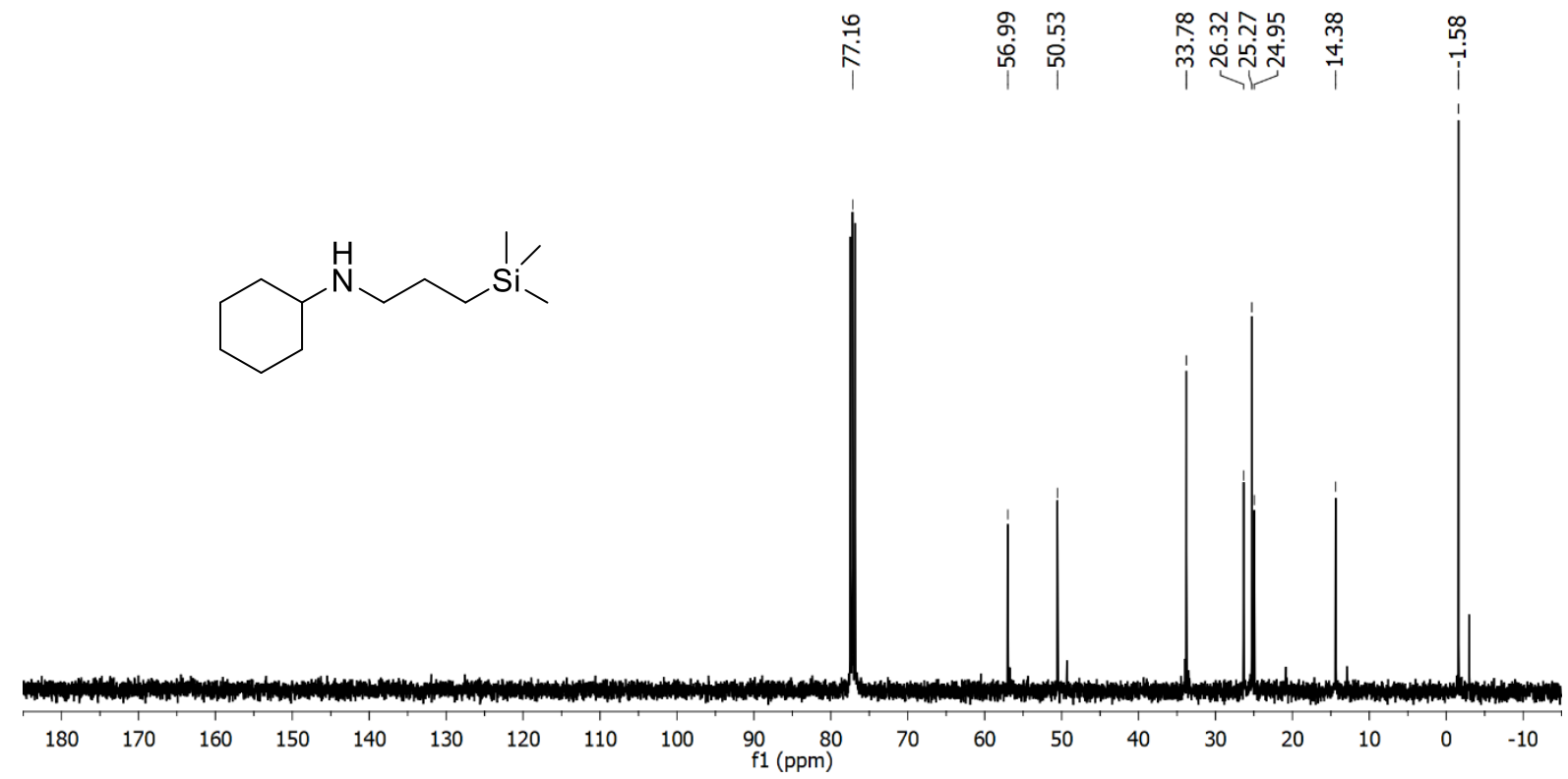

Figure S40. ${ }^{13} \mathrm{C}$ NMR spectrum $\left(100 \mathrm{MHz}, \mathrm{CDCl}_{3}, 298 \mathrm{~K}\right)$ of $\mathrm{N}$-(3-(trimethylsilyl)propyl)cyclohexanamine (12). 


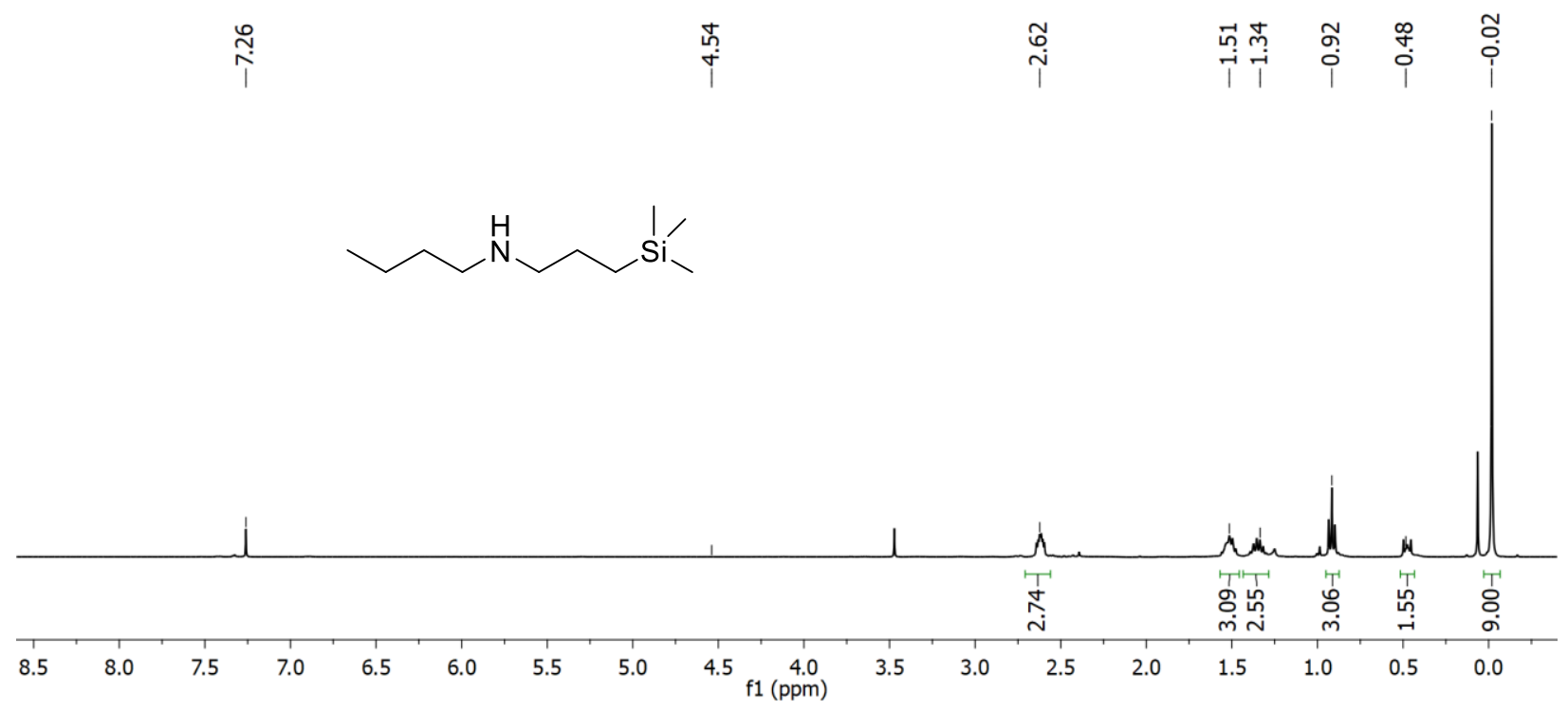

Figure S41. ${ }^{1} \mathrm{H}$ NMR spectrum $\left(400 \mathrm{MHz}, \mathrm{CDCl}_{3}, 298 \mathrm{~K}\right)$ of $\mathrm{N}$-(3-(trimethylsilyl)propyl)butan-1-amine (13).

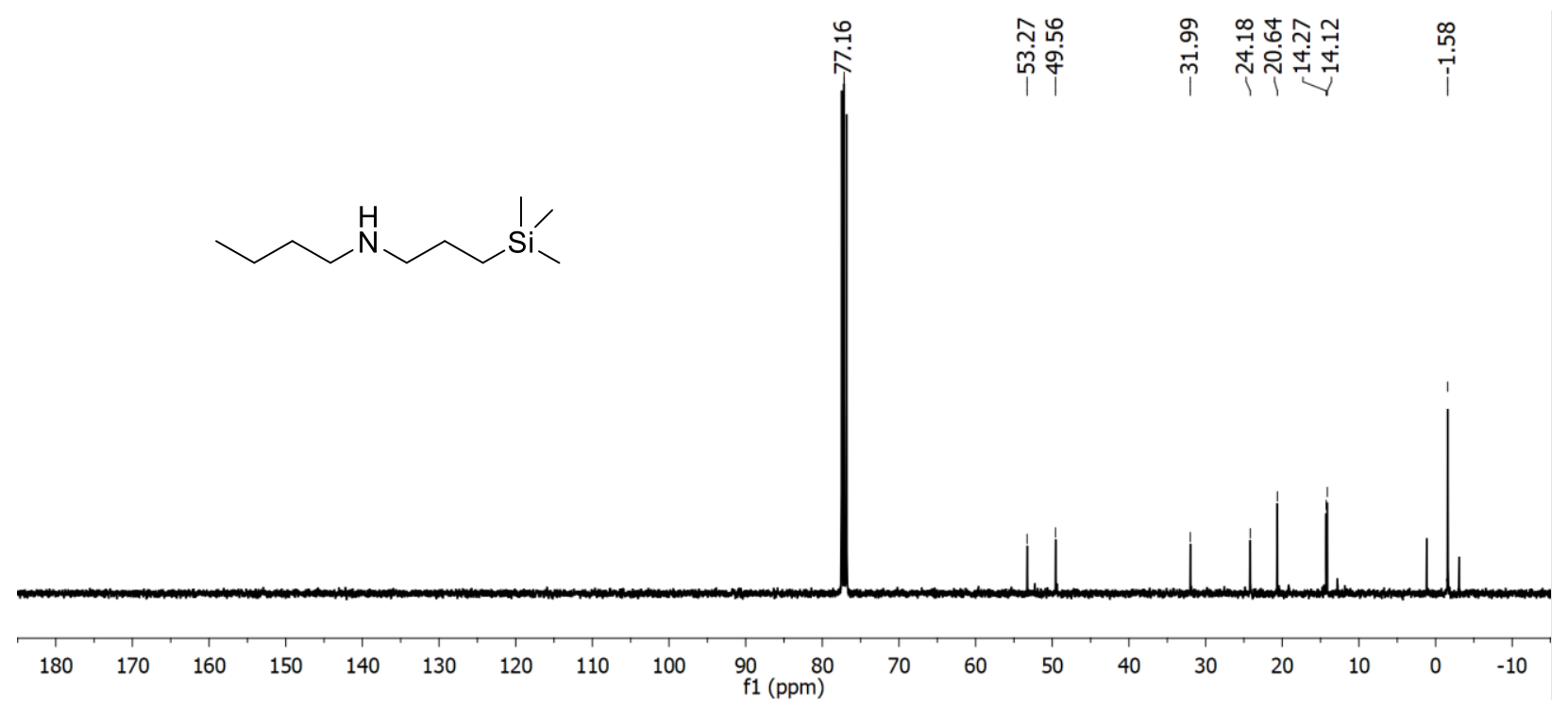

Figure S42. ${ }^{13} \mathrm{C}$ NMR spectrum (100 MHz, $\mathrm{CDCl}_{3}, 298 \mathrm{~K}$ ) of $\mathrm{N}$-(3-(trimethylsilyl)propyl)butan-1-amine (13). 
<smiles>CC(CNC1CCCCC1)C[Si](C)(C)C</smiles>

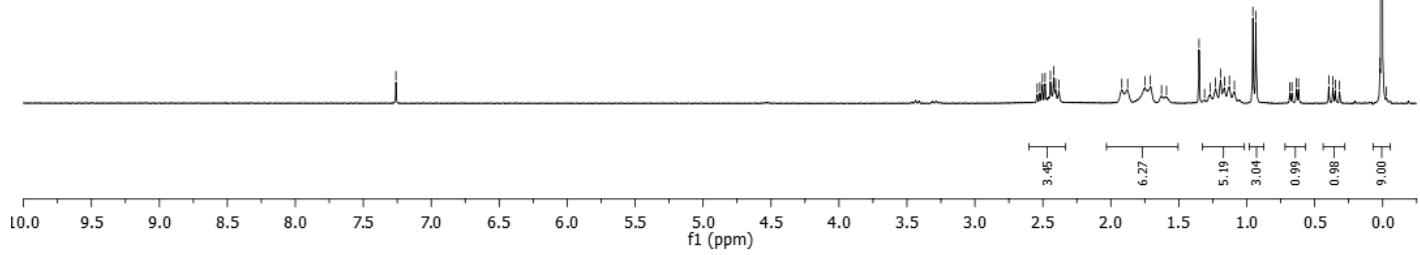

Figure S43. ${ }^{1} \mathrm{H}$ NMR spectrum $\left(300 \mathrm{MHz}, \mathrm{CDCl}_{3}, 298 \mathrm{~K}\right)$ of $\mathrm{N}$-(2-methyl-3-(trimethylsilyl)propyl)cyclohexanamine (14).
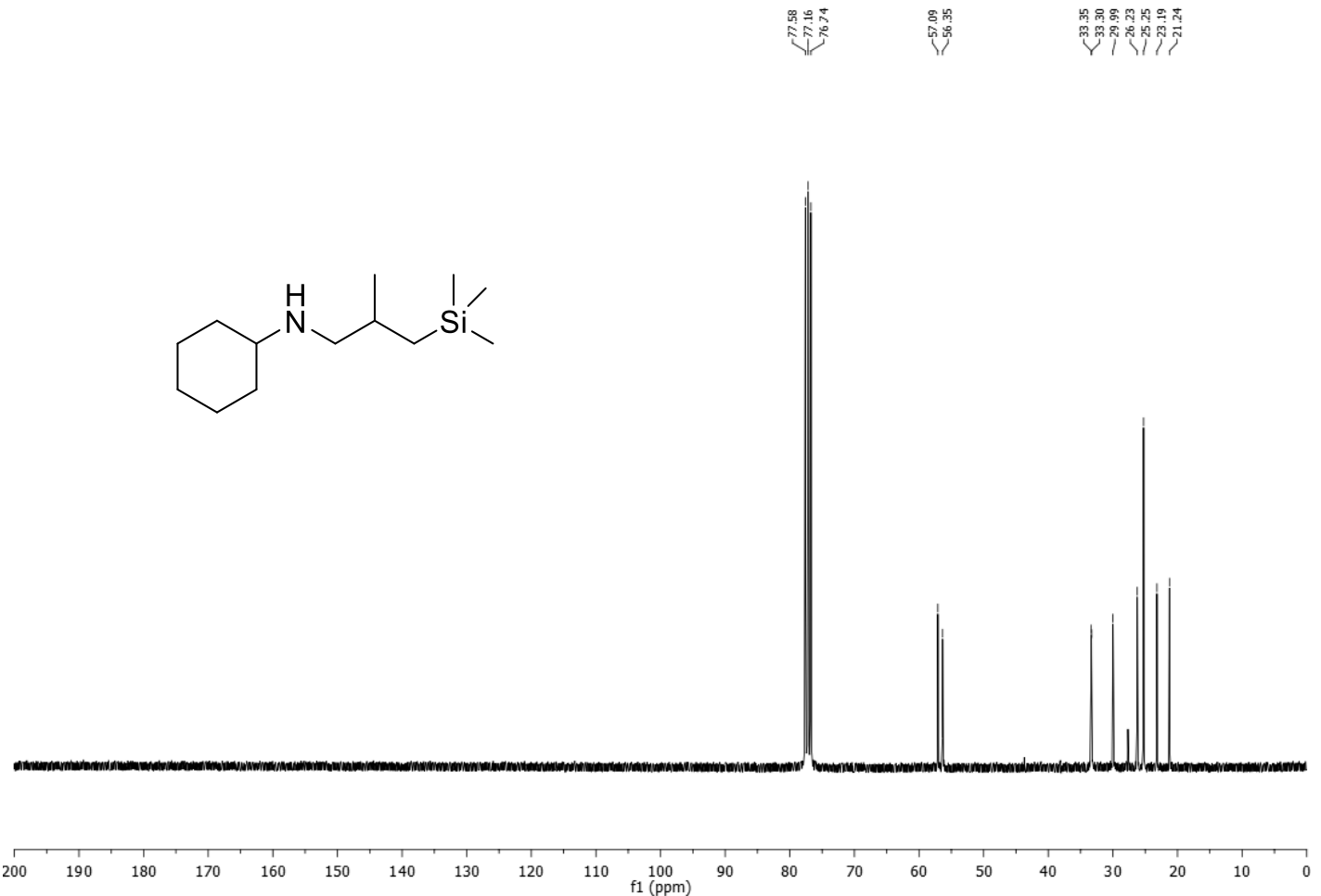

Figure S44. ${ }^{13} \mathrm{C}$ NMR spectrum $\left(75 \mathrm{MHz}, \mathrm{CDCl}_{3}, 298 \mathrm{~K}\right)$ of $\mathrm{N}$-(2-methyl-3-(trimethylsilyl)propyl)cyclohexanamine (14). 

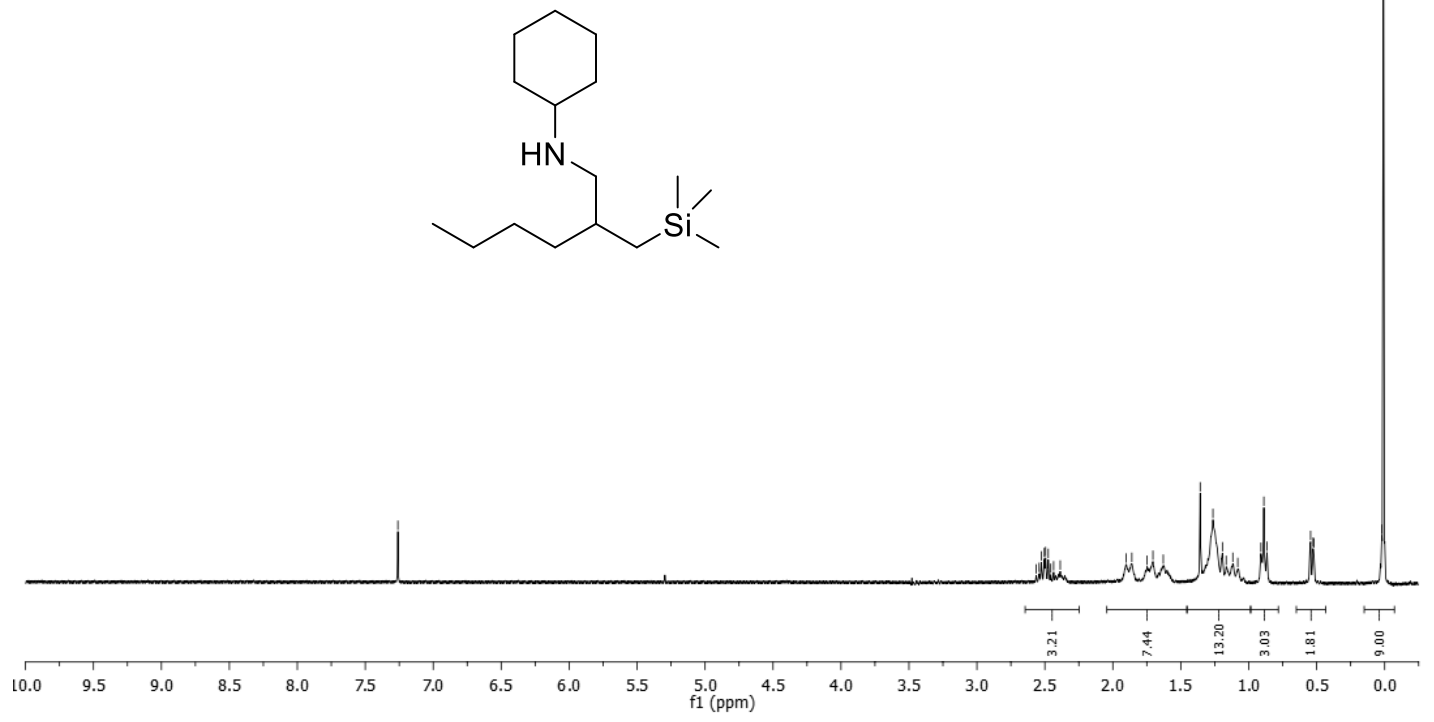

Figure S45. ${ }^{1} \mathrm{H}$ NMR spectrum $\left(300 \mathrm{MHz}, \mathrm{CDCl}_{3}, 298 \mathrm{~K}\right)$ of $\mathrm{N}-(2-(($ trimethylsilyl)methyl)hexyl)cyclohexanamine (15).

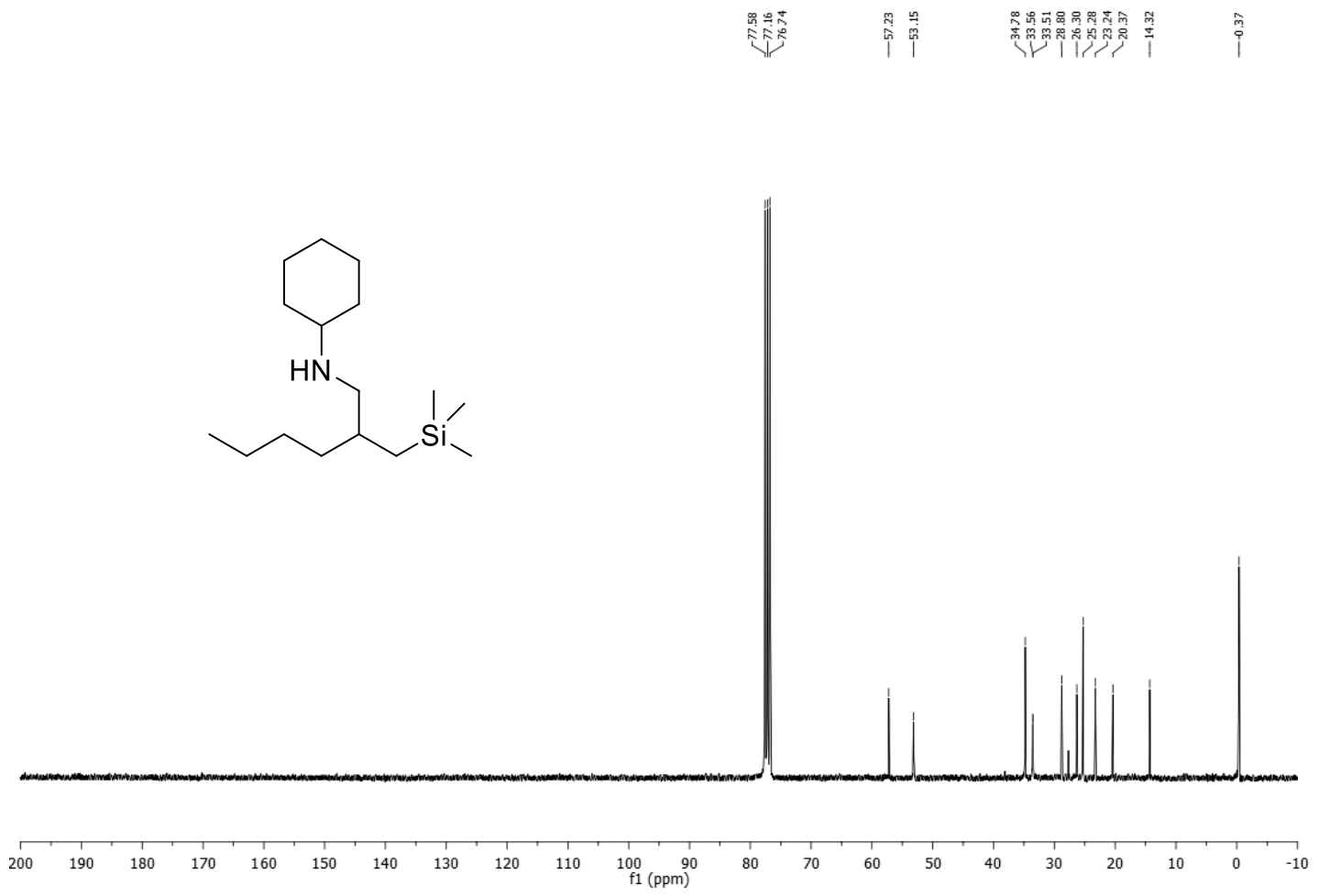

Figure S46. ${ }^{13} \mathrm{C}$ NMR spectrum $\left(75 \mathrm{MHz} \mathrm{CDCl}_{3}, 298 \mathrm{~K}\right)$ of $\mathrm{N}-(2-(($ trimethylsilyl)methyl)hexyl)cyclohexanamine (15). 


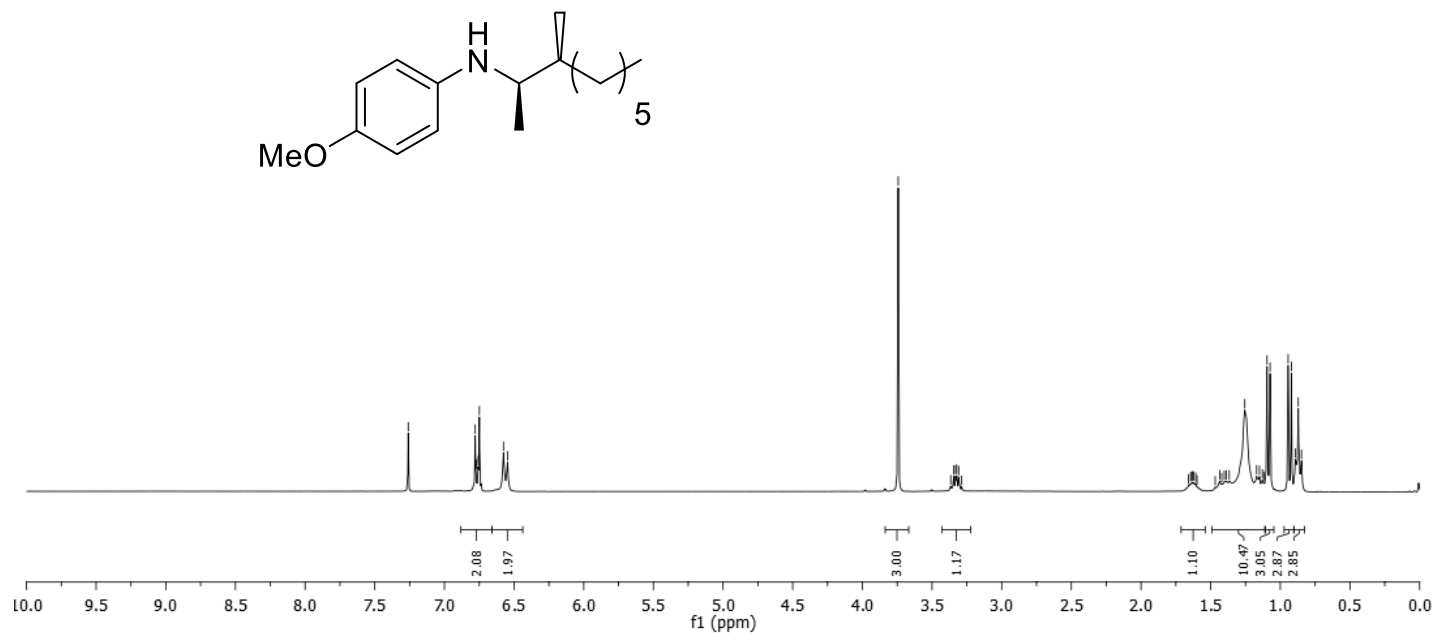

Figure S47. ${ }^{1} \mathrm{H}$ NMR spectrum (300 MHz, $\mathrm{CDCl}_{3}, 298 \mathrm{~K}$ ) of 4-methoxy- $N$-(3-methylnonan-2-yl)aniline (16).

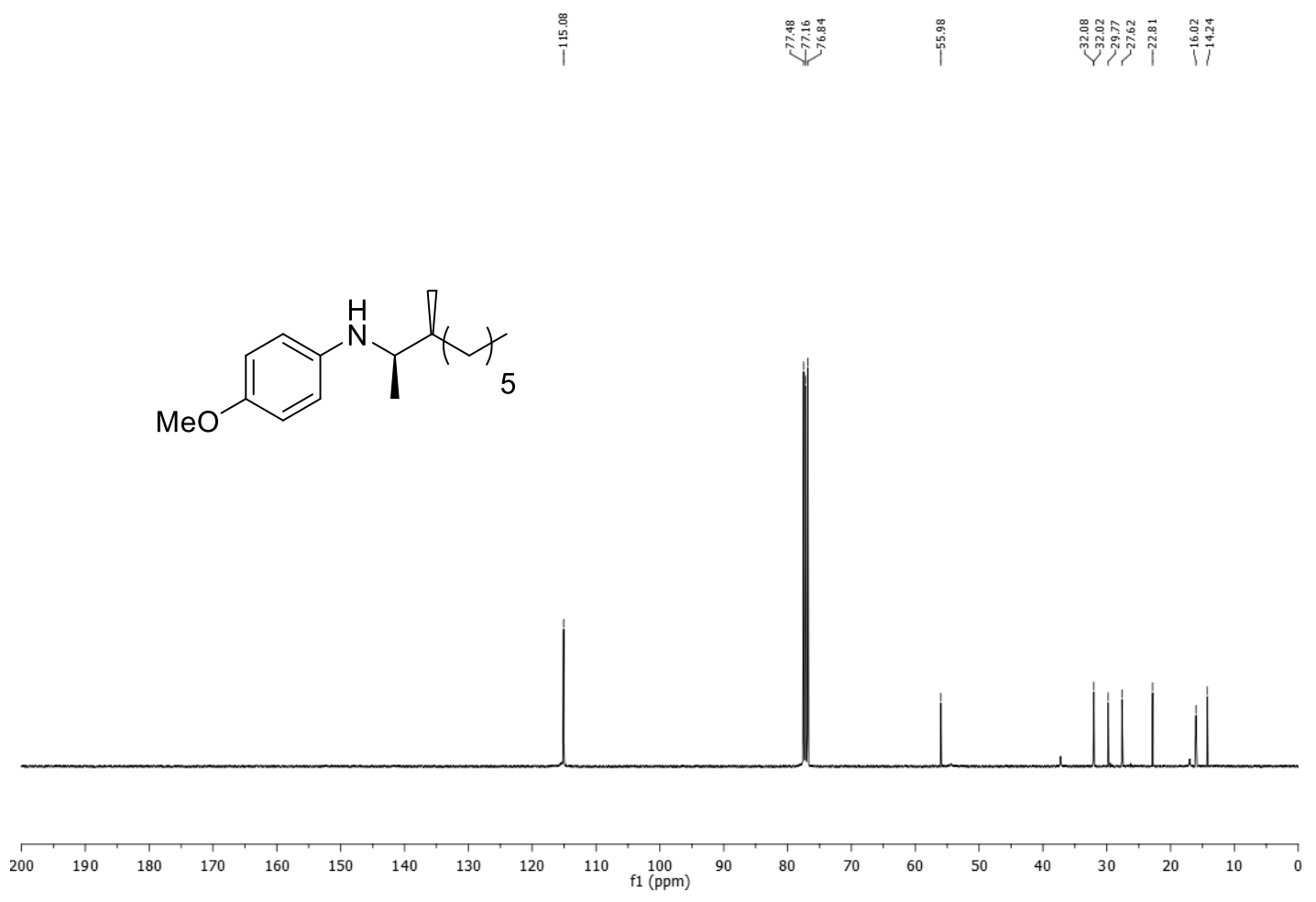

Figure S48. ${ }^{13} \mathrm{C}$ NMR spectrum $\left(75 \mathrm{MHz}, \mathrm{CDCl}_{3}, 298 \mathrm{~K}\right)$ of 4-methoxy- $\mathrm{N}$-(3-methylnonan-2-yl)aniline (16). 


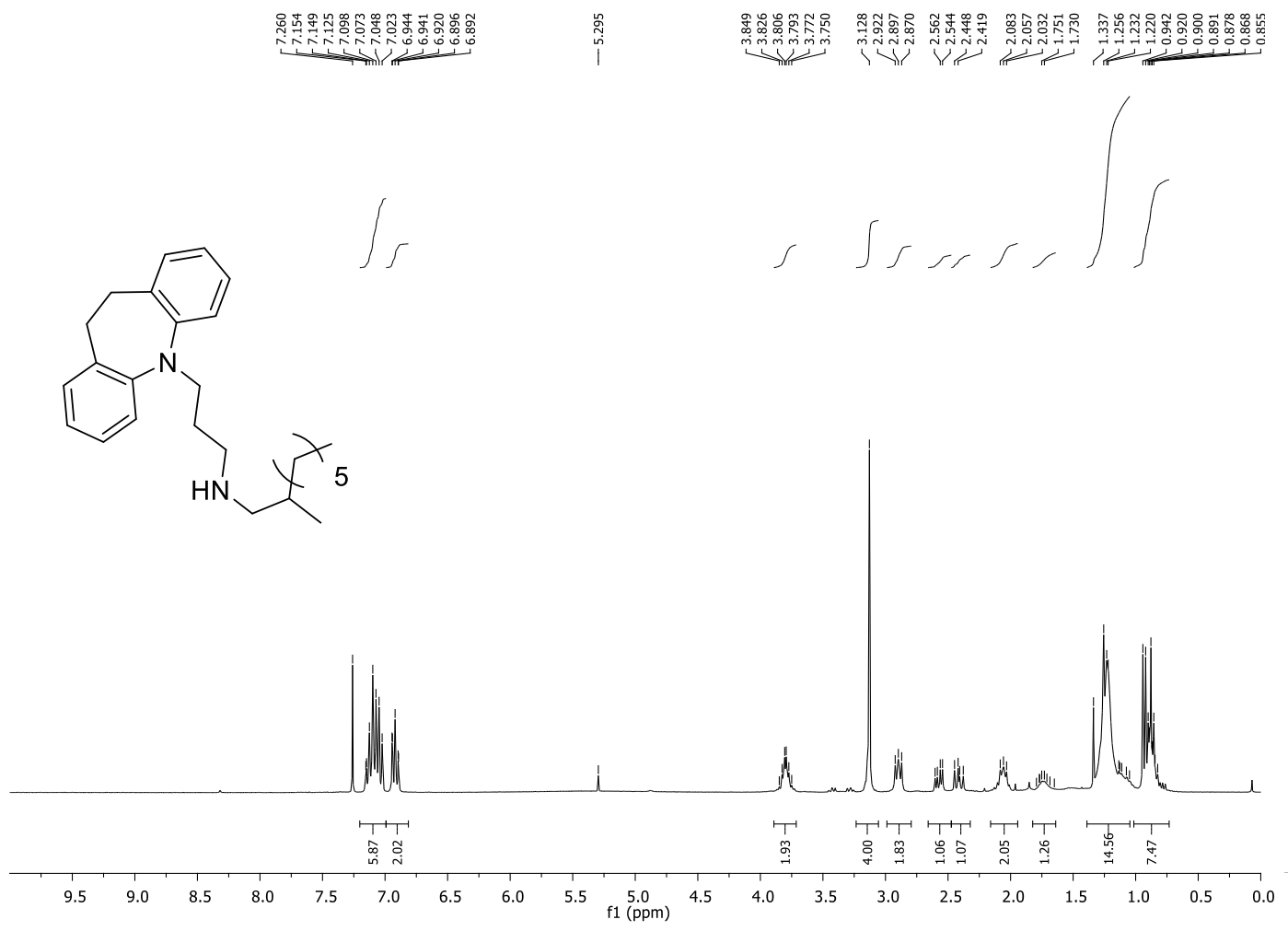

Figure S49. ${ }^{1} \mathrm{H}$ NMR spectrum $\left(300 \mathrm{MHz}, \mathrm{CDCl}_{3}, 298 \mathrm{~K}\right)$ of Desipramine- $N$-(2-methyl)octylamine (19).

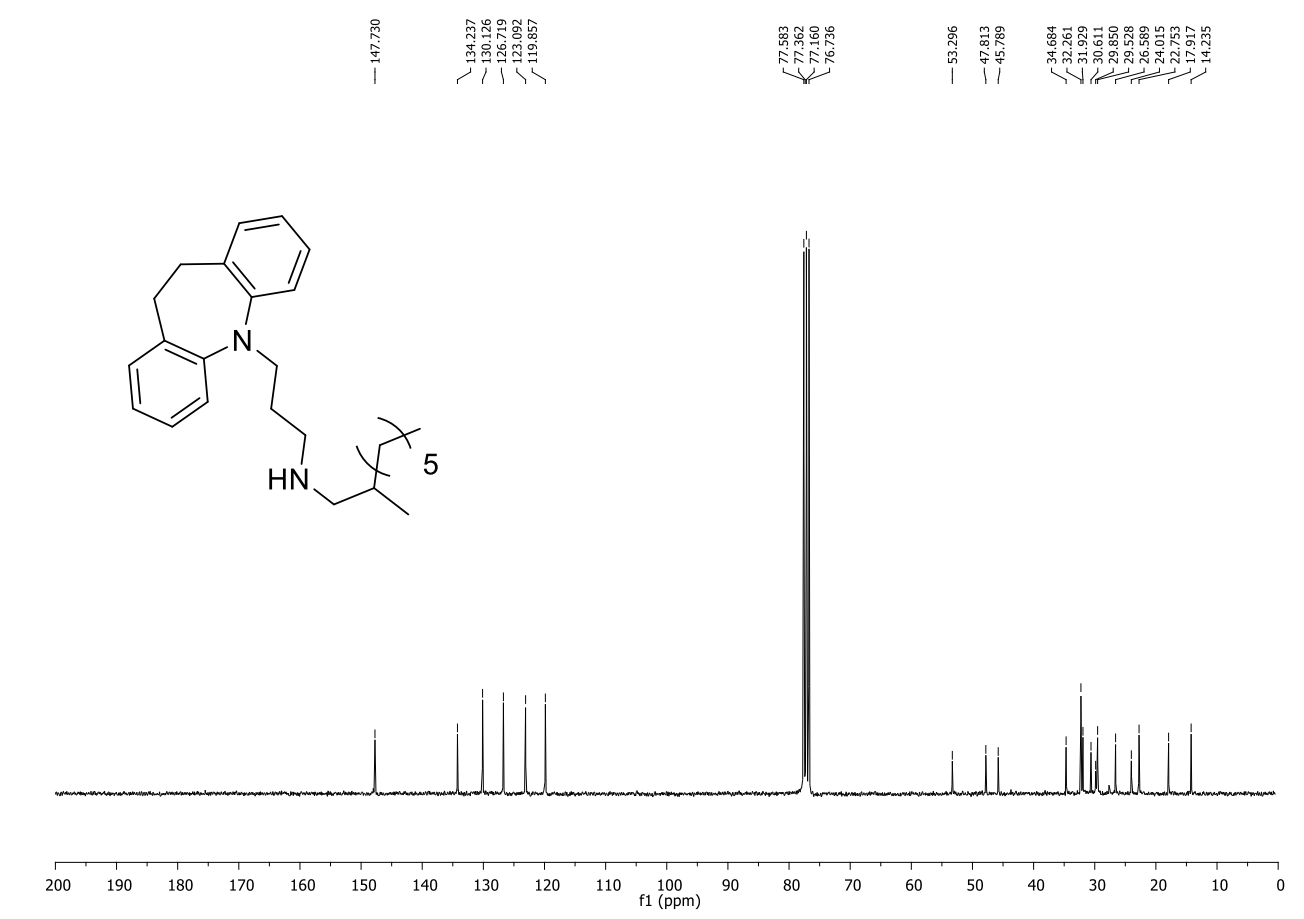

Figure S50. ${ }^{13} \mathrm{C}$ NMR spectrum $\left(75 \mathrm{MHz}, \mathrm{CDCl}_{3}, 298 \mathrm{~K}\right.$ ) of Desipramine- $\mathrm{N}$-(2-methyl)octylamine (19). 

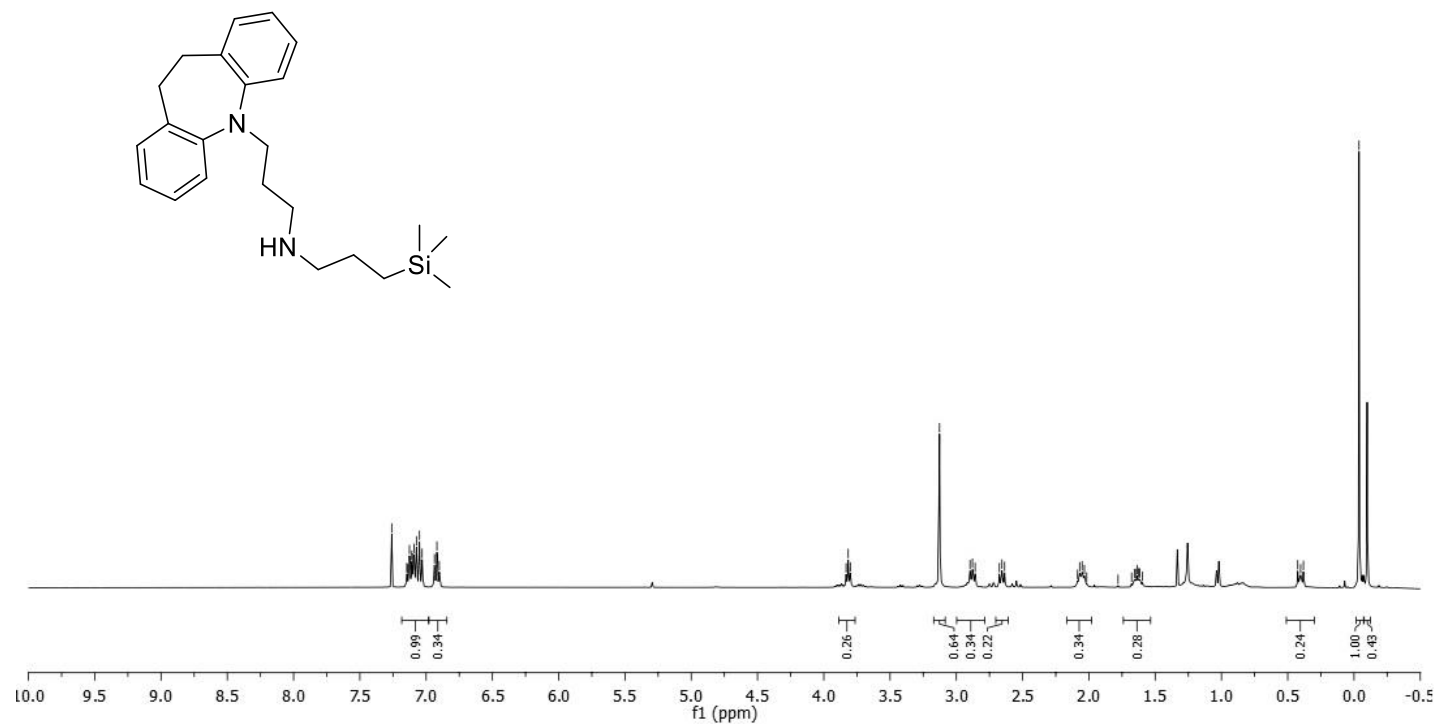

Figure S51. ${ }^{1} \mathrm{H}$ NMR spectrum (300 MHz, CDCl $\left.3,298 \mathrm{~K}\right)$ of Desipramine- $N$-(3-(trimethylsilyl)propylamine (20). (7:3 (linear:branched))

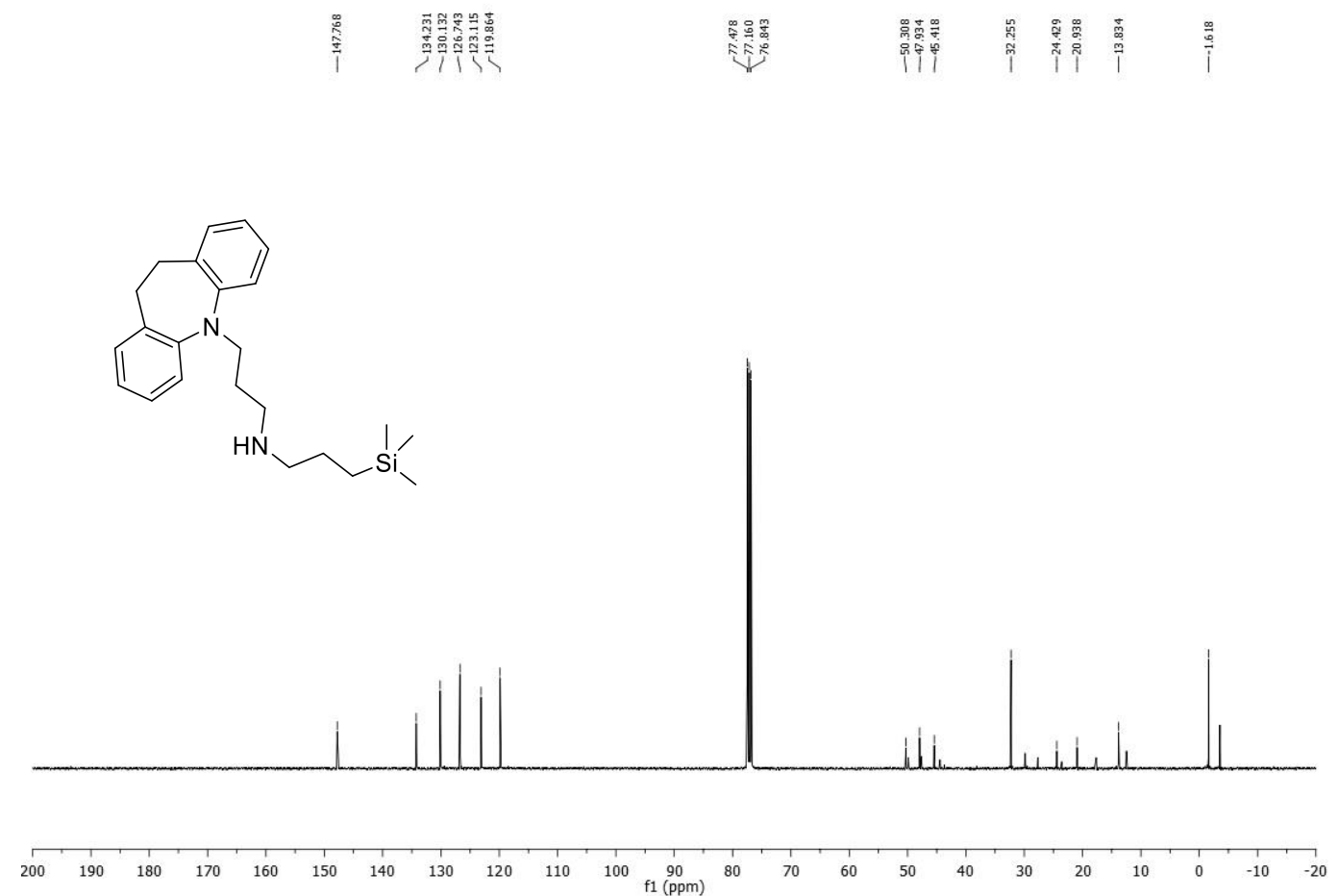

Figure S52. ${ }^{13} \mathrm{C}$ NMR spectrum (75 MHz, $\mathrm{CDCl}_{3}, 298 \mathrm{~K}$ ) of Desipramine- $\mathrm{N}$-(3-(trimethylsilyl)propylamine (20). (7:3 (linear:branched)) 

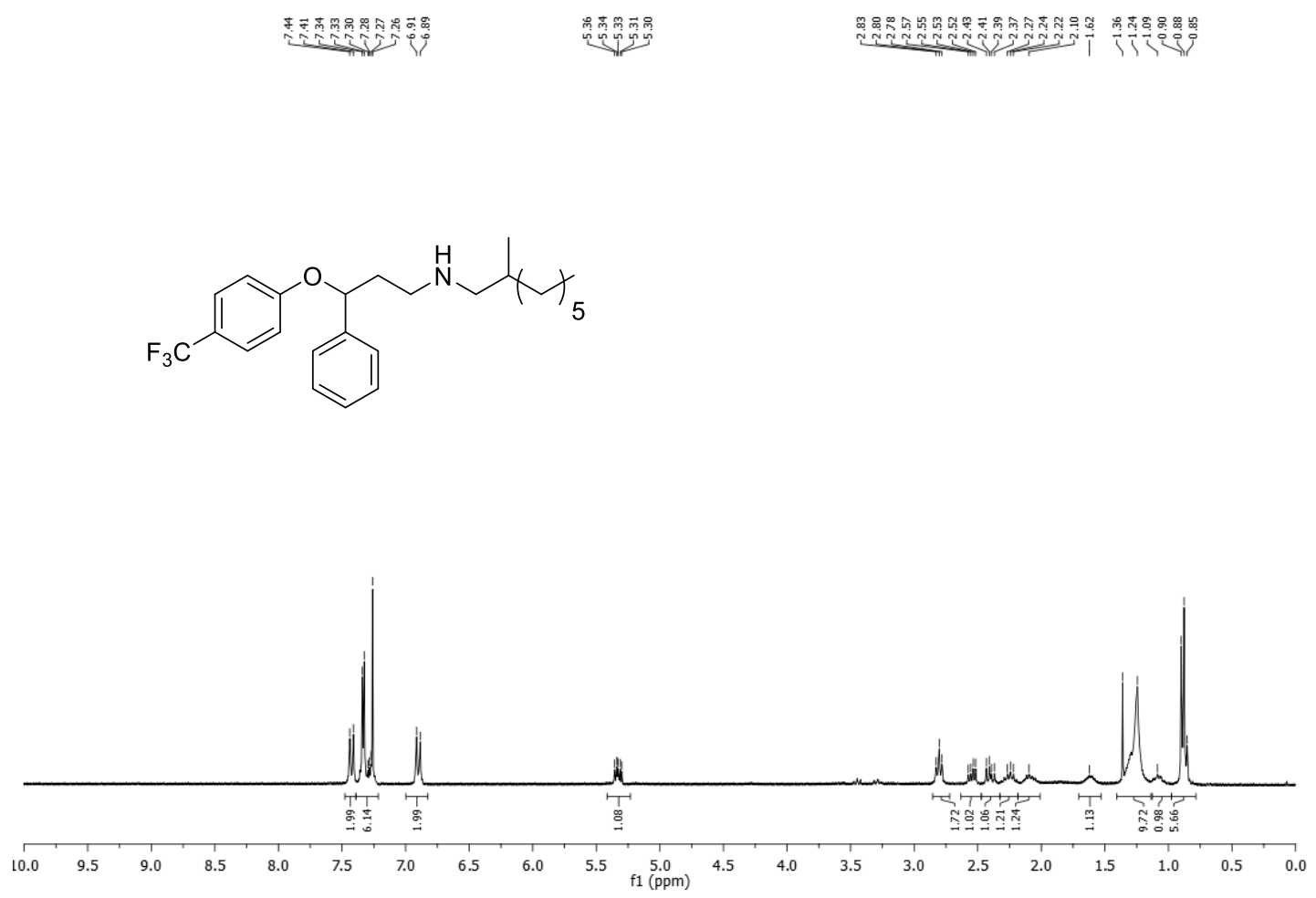

Figure S53. ${ }^{1} \mathrm{H}$ NMR spectrum ( $300 \mathrm{MHz}, \mathrm{CDCl}_{3}, 298 \mathrm{~K}$ ) of Fluoxetine- $\mathrm{N}$-(2-methyl)octylamine (22).

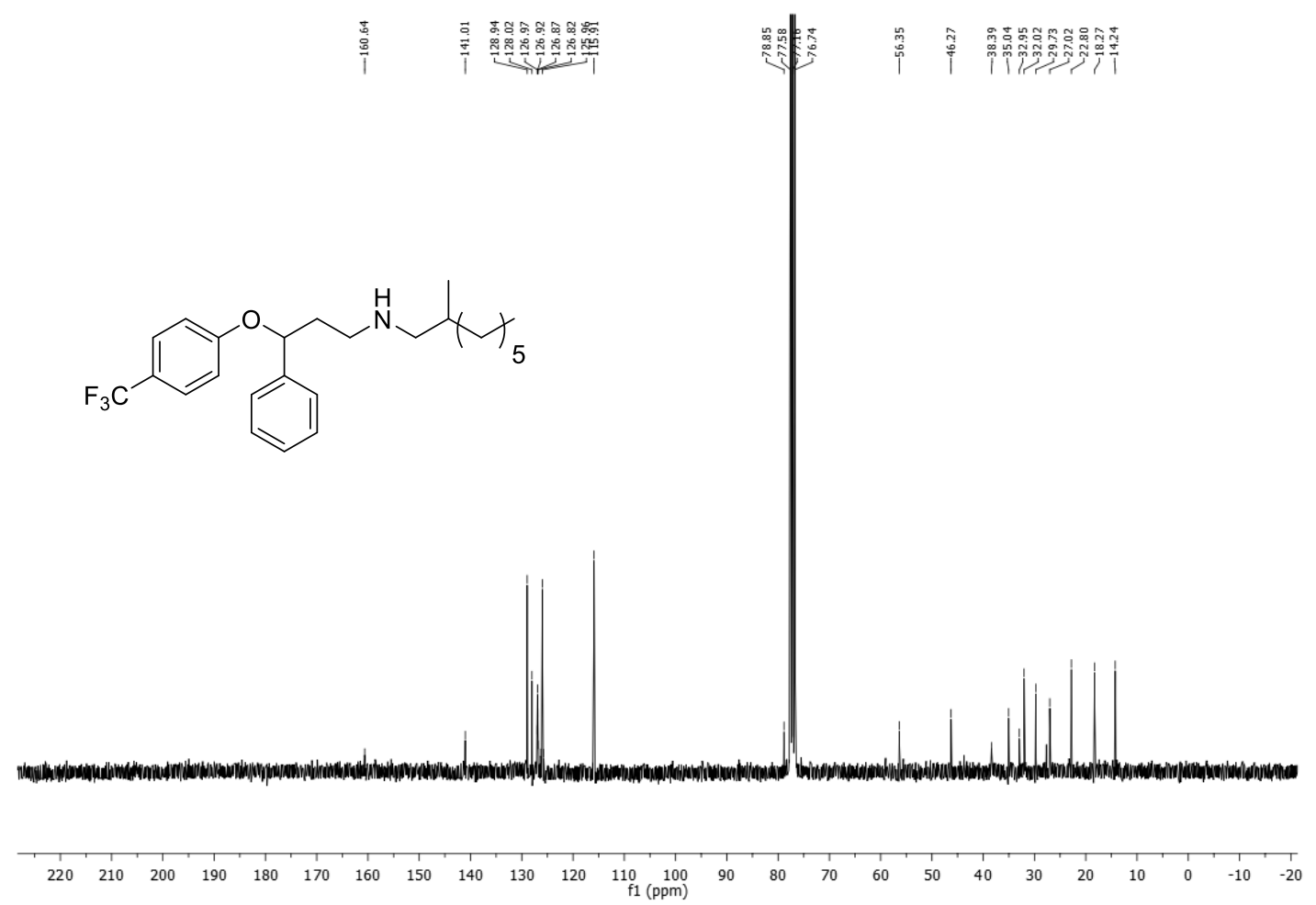

Figure S54. ${ }^{13} \mathrm{C}$ NMR spectrum $\left(75 \mathrm{MHz}, \mathrm{CDCl}_{3}, 298 \mathrm{~K}\right)$ of Fluoxetine- $\mathrm{N}$-(2-methyl)octylamine (22). 
<smiles>CC(C)(C)CNCCC(Oc1ccc(C(F)(F)F)cc1)c1ccccc1</smiles>

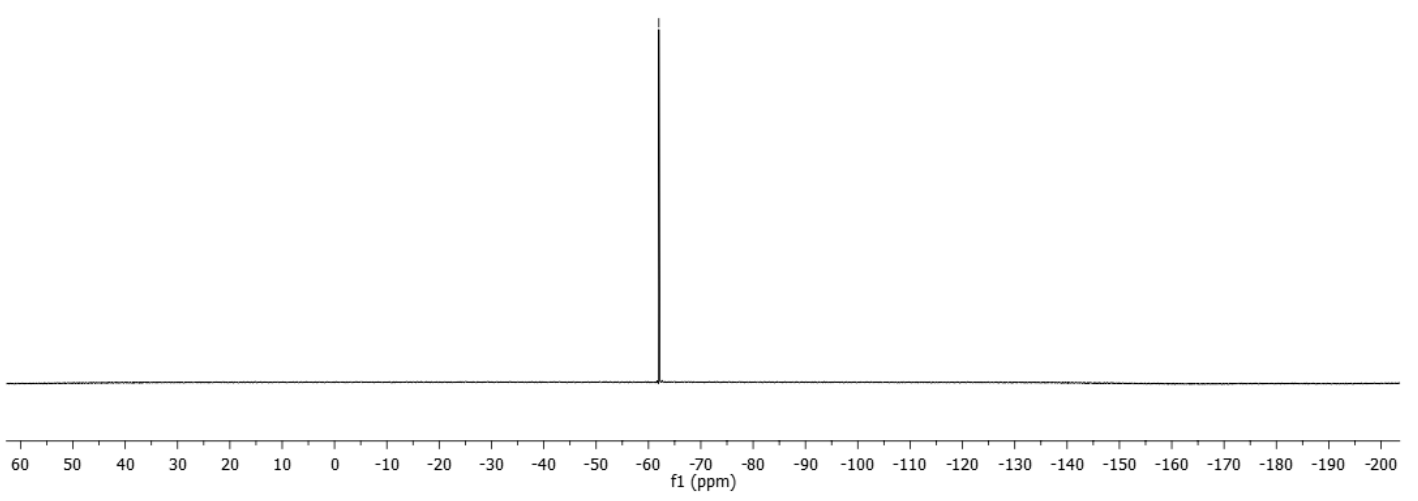

Figure S55. ${ }^{19} \mathrm{~F} \mathrm{NMR}$ spectrum (282 MHz, $\left.\mathrm{CDCl}_{3}, 298 \mathrm{~K}\right)$ of Fluoxetine- $\mathrm{N}$-(2-methyl)octylamine (22).
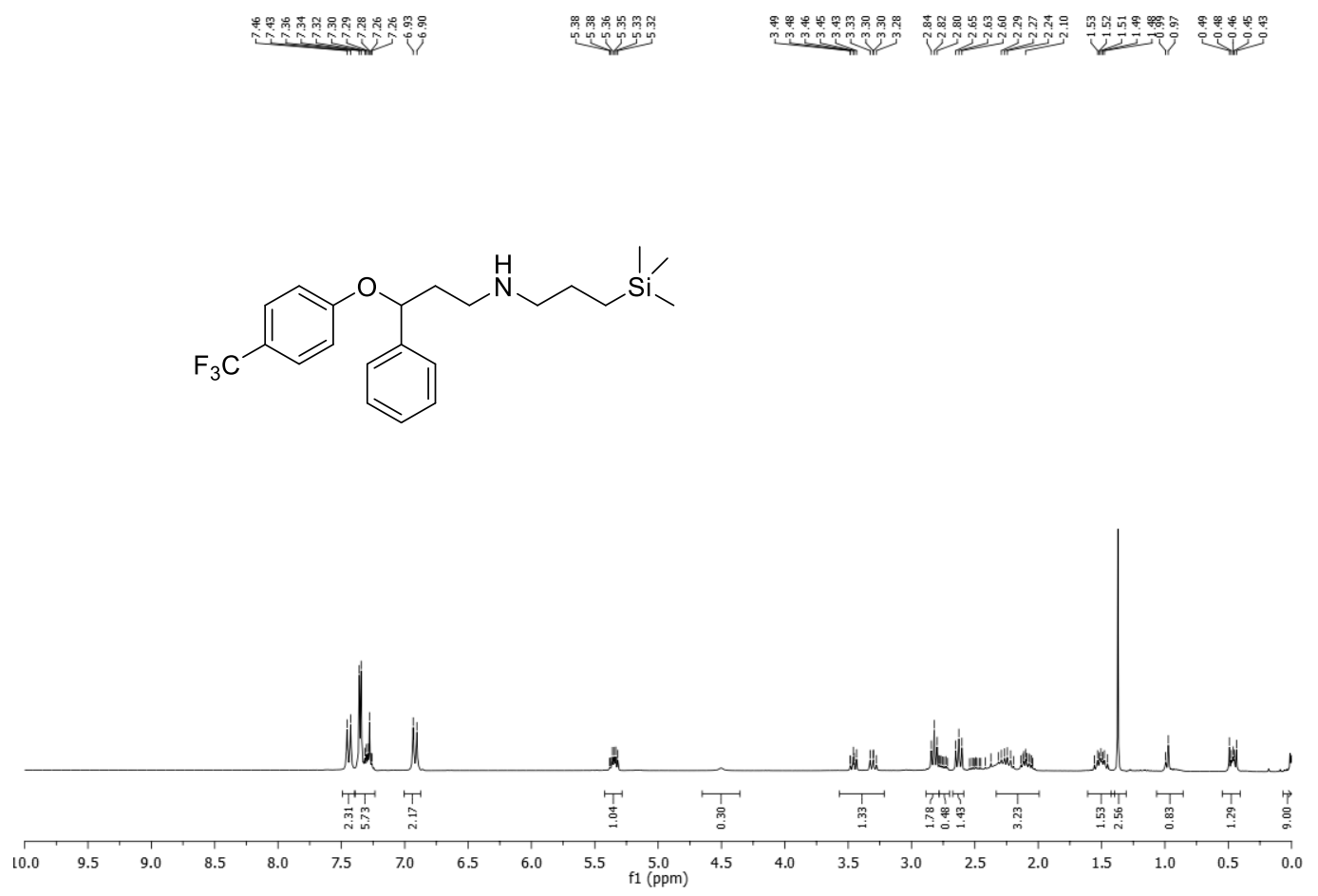

Figure S56. ${ }^{1} \mathrm{H}$ NMR spectrum $\left(300 \mathrm{MHz}, \mathrm{CDCl}_{3}, 298 \mathrm{~K}\right.$ ) of Fluoxetine- $\mathrm{N}$-(3-(trimethylsilyl)propylamine (23), contaminated with L2H. (7:3 (linear:branched)) 


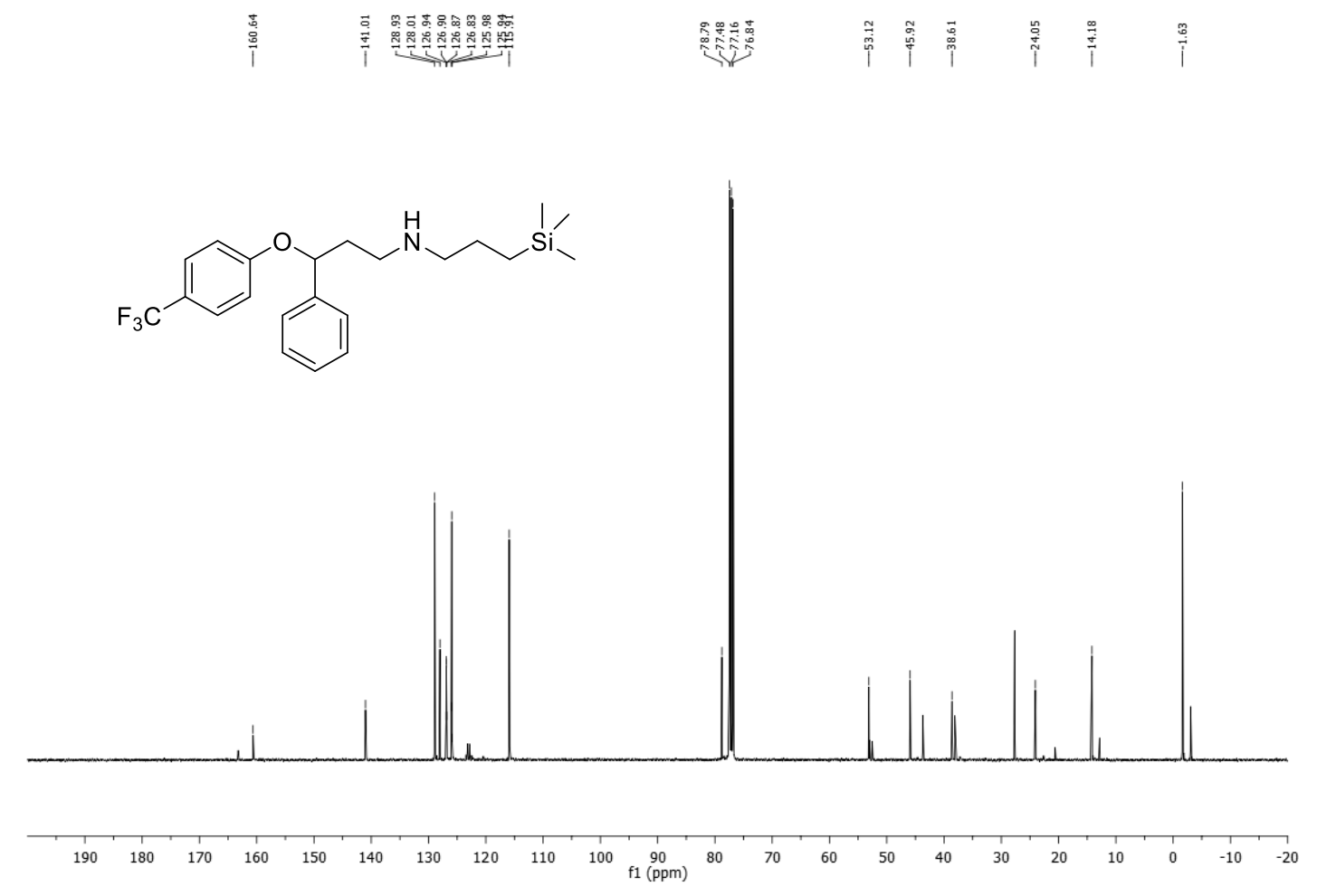

Figure S57. ${ }^{13} \mathrm{C}$ NMR spectrum (100 MHz, $\mathrm{CDCl}_{3}, 298 \mathrm{~K}$ ) of Fluoxetine- $\mathrm{N}$-(3-(trimethylsilyl)propylamine (23), contaminated with L2H. (7:3 (linear:branched))

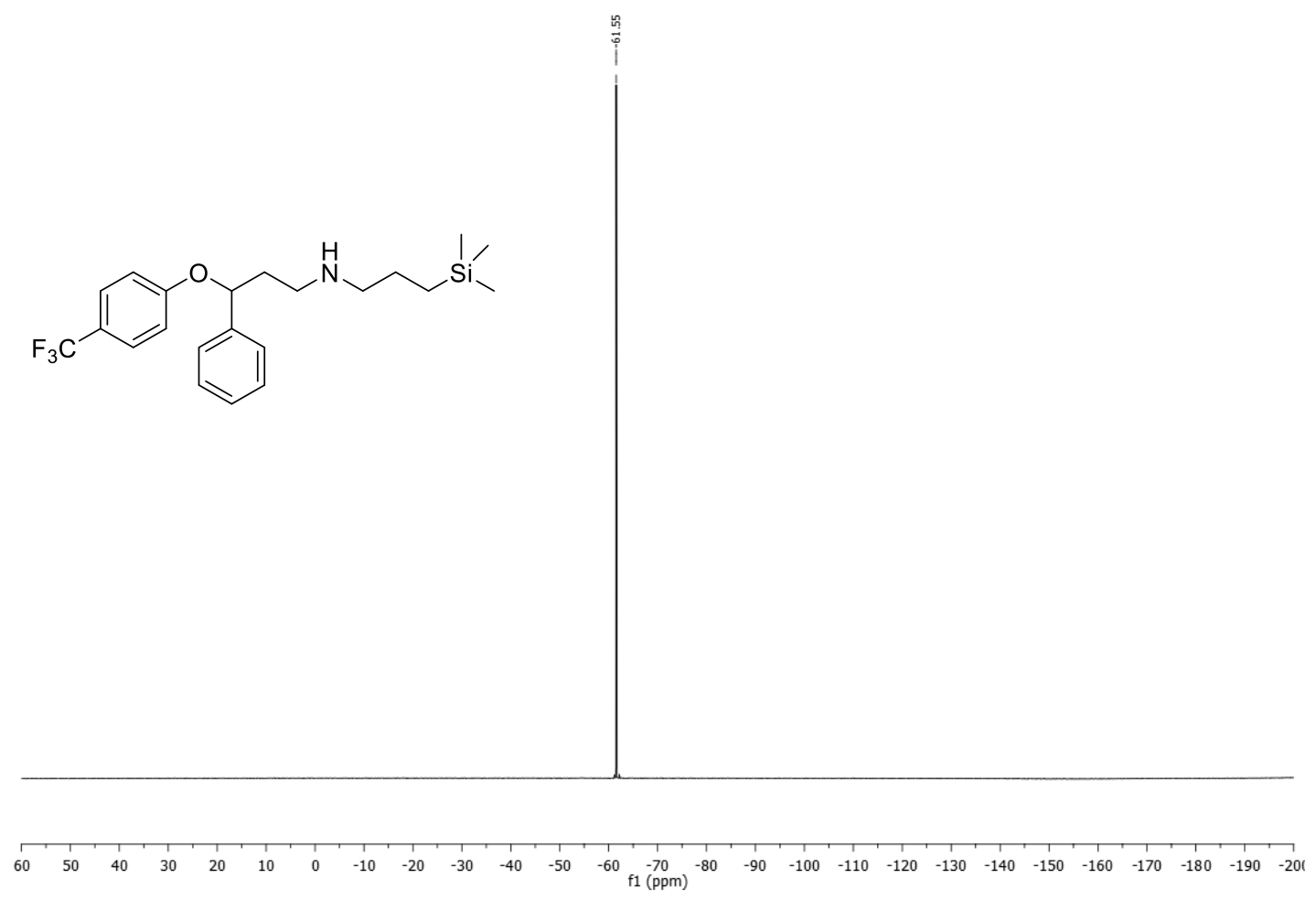

Figure S58. ${ }^{19} \mathrm{~F}$ NMR spectrum (282 MHz, $\mathrm{CDCl}_{3}, 298 \mathrm{~K}$ ) of Fluoxetine- $N$-(3-(trimethylsilyl)propylamine (23). 


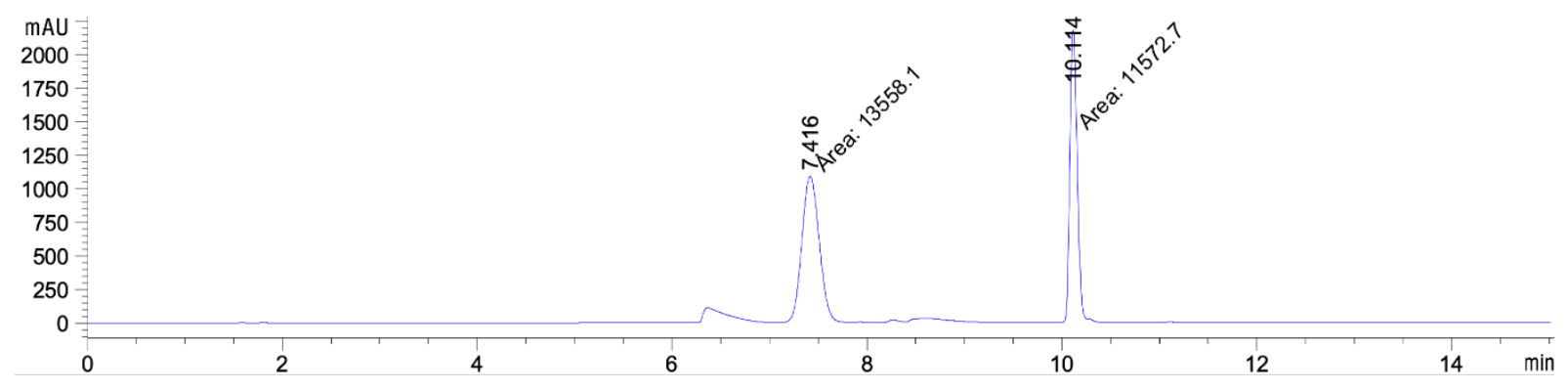

Figure S59. Crude mixture of hydroaminoalkylation of Fluoxetine $(0.25 \mathrm{mmol})$, vinylsilane $(0.37 \mathrm{mmol})$ with $5 \mathrm{~mol} \%$ of L2Ta $\left(\mathrm{CH}_{2} \mathrm{SiMe}_{3}\right)_{3} \mathrm{Cl}$ in $0.5 \mathrm{~mL}$ of $d_{8}$-toluene at $110{ }^{\circ} \mathrm{C}$ for $3 \mathrm{~h}$. HPLC-MS, 10 to $90 \%$ acetonitrile [0.1\% formic acid (FA)] in water $(0.1 \% \mathrm{FA})$ over $10 \mathrm{~min}$ followed by $90 \%$ acetonitrile $(0.1 \% \mathrm{FA})$ in water $(0.1 \% \mathrm{FA})$ over $5 \mathrm{~min}$, flow rate of $1 \mathrm{~mL} / \mathrm{min}$ on an Eclipse XDB-C18, $5 \mu \mathrm{m}, 4.6 \times 150 \mathrm{~mm}, \lambda=263 \mathrm{~nm}$, (Fluoxetine, $\mathrm{t}_{\mathrm{R}}=7.42 \mathrm{~min}$, area $\left.=54 \%,[\mathrm{M}+\mathrm{H}]^{+}=310\right),\left(\mathbf{2 3}, \mathrm{t}_{\mathrm{R}}=\right.$ $10.11 \mathrm{~min}$, area $\left.=46 \%,[\mathrm{M}+\mathrm{H}]^{+}=410\right)$ 

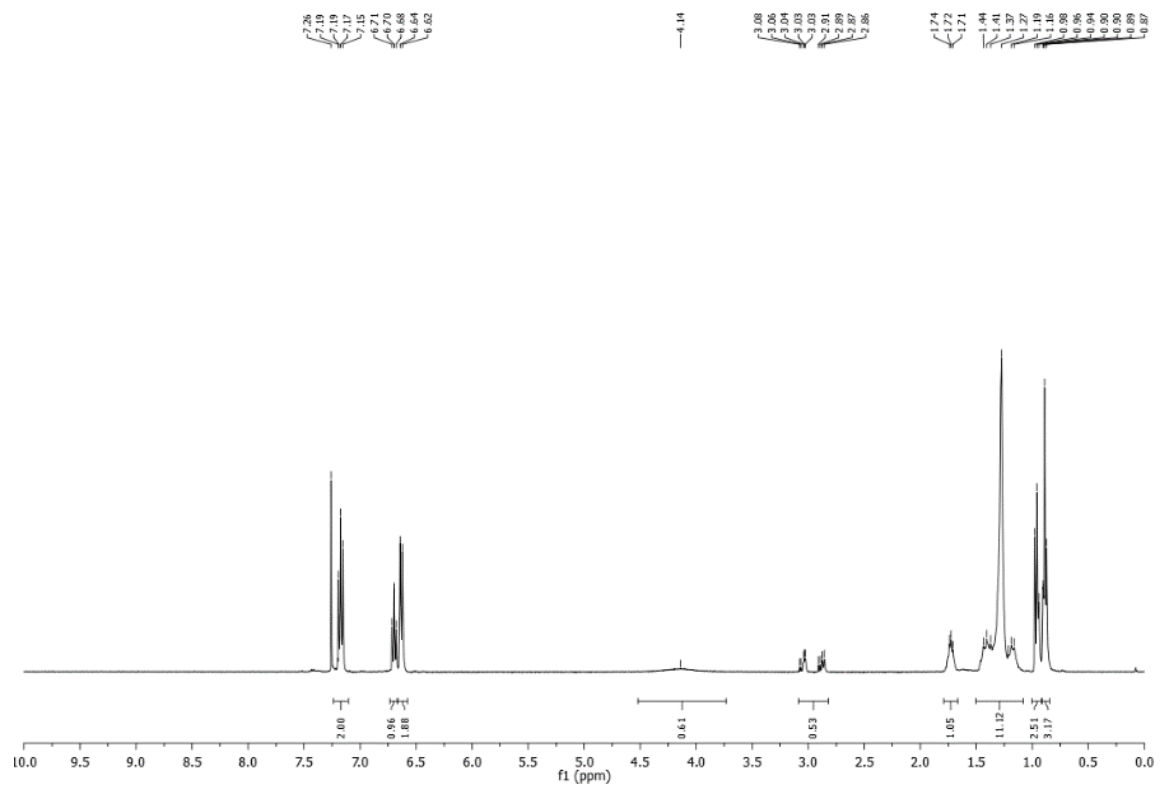

Figure S60. ${ }^{1} \mathrm{H}$ NMR spectrum $\left(300 \mathrm{MHz}, \mathrm{CDCl}_{3}, 298 \mathrm{~K}\right)$ of deuterium labeling experiment.

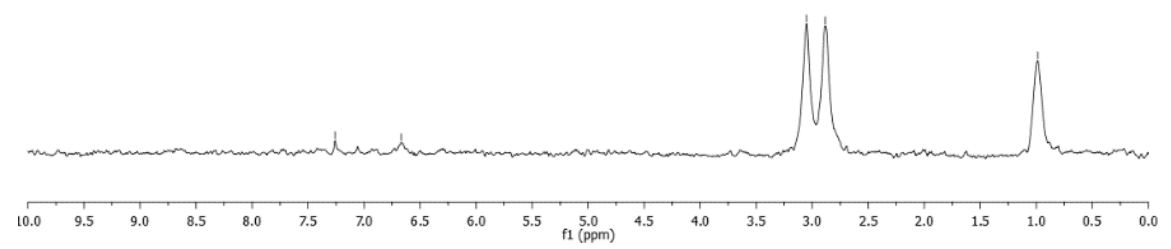

Figure S61. ${ }^{2} \mathrm{H}$ NMR spectrum $\left(400 \mathrm{MHz}, \mathrm{CHCl}_{3}, 298 \mathrm{~K}\right)$ of deuterium labeling experiment. 


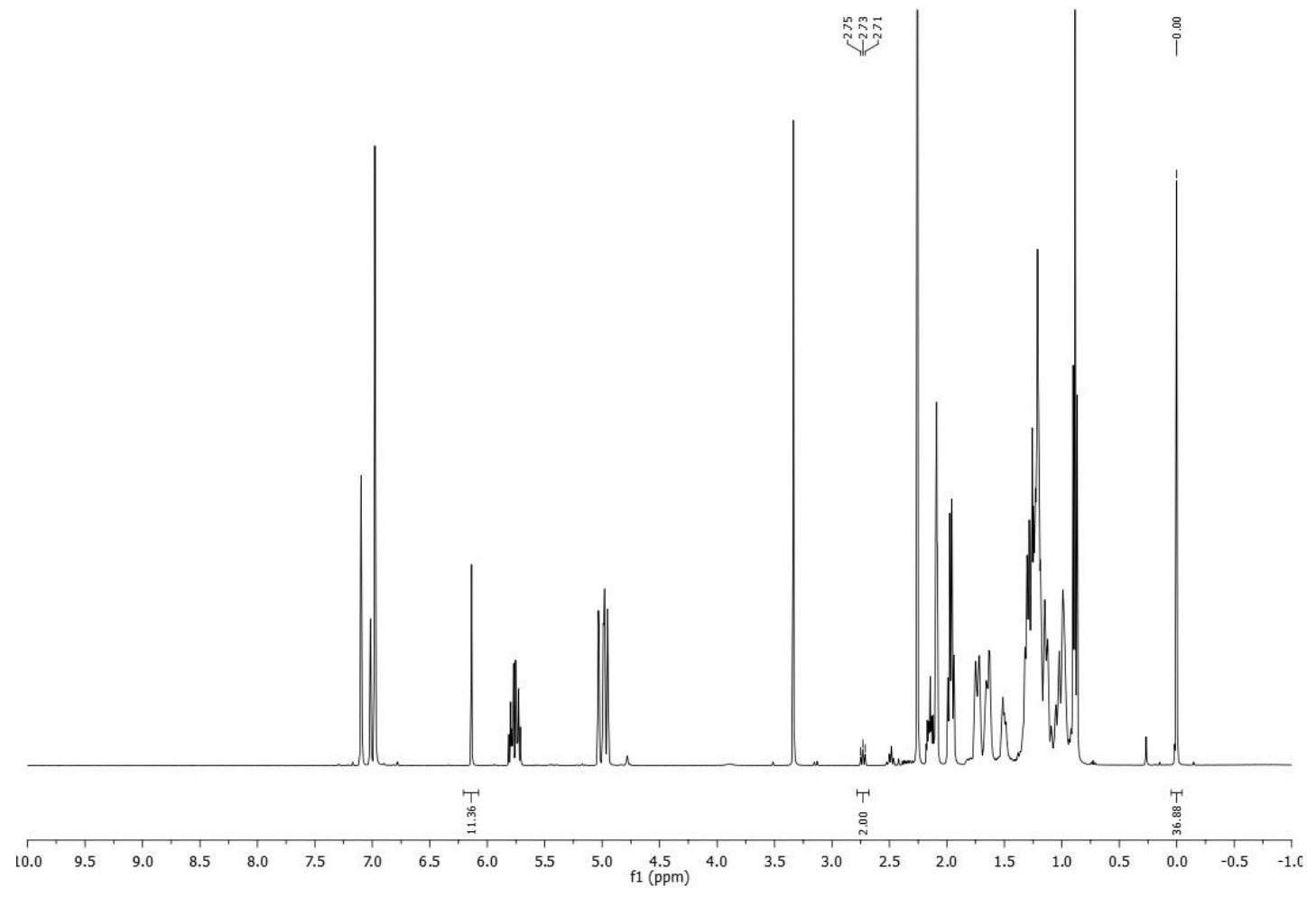

Figure S62. ${ }^{1} \mathrm{H}$ NMR ( $d_{8}$-toluene, $\left.400 \mathrm{MHz}, 298 \mathrm{~K}\right)$, L2Ta $\left(\mathrm{CH}_{2} \mathrm{SiMe}_{3}\right)_{3} \mathrm{Cl}(5 \mathrm{~mol} \%)$ in the presence of 1 equivalent of $\mathrm{N}$ methylcyclohexylamine and 1.5 equivalents of 1 -octene heated at $105{ }^{\circ} \mathrm{C}$ for $5 \mathrm{~min}$.
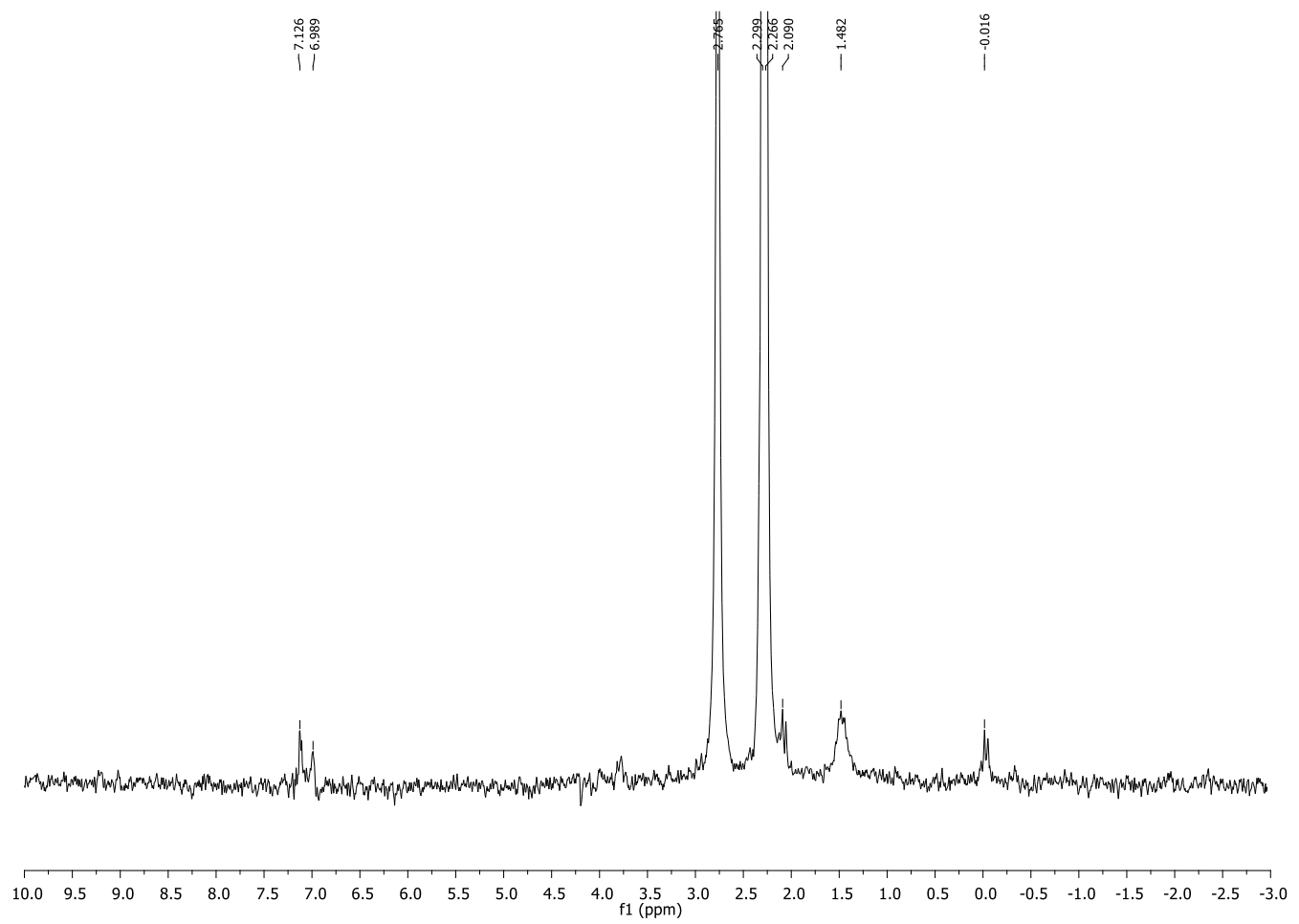

Figure S63. ${ }^{2} \mathrm{H}$ NMR (toluene, $400 \mathrm{MHz}, 298 \mathrm{~K}$ ), $\mathrm{L} 2 \mathrm{Ta}\left(\mathrm{CH}_{2} \mathrm{SiMe}_{3}\right)_{3} \mathrm{Cl}$ with 20 equivalents of $\mathrm{PhNHCD}_{3}$ at $105{ }^{\circ} \mathrm{C}$ for $1 \mathrm{~h}$. 


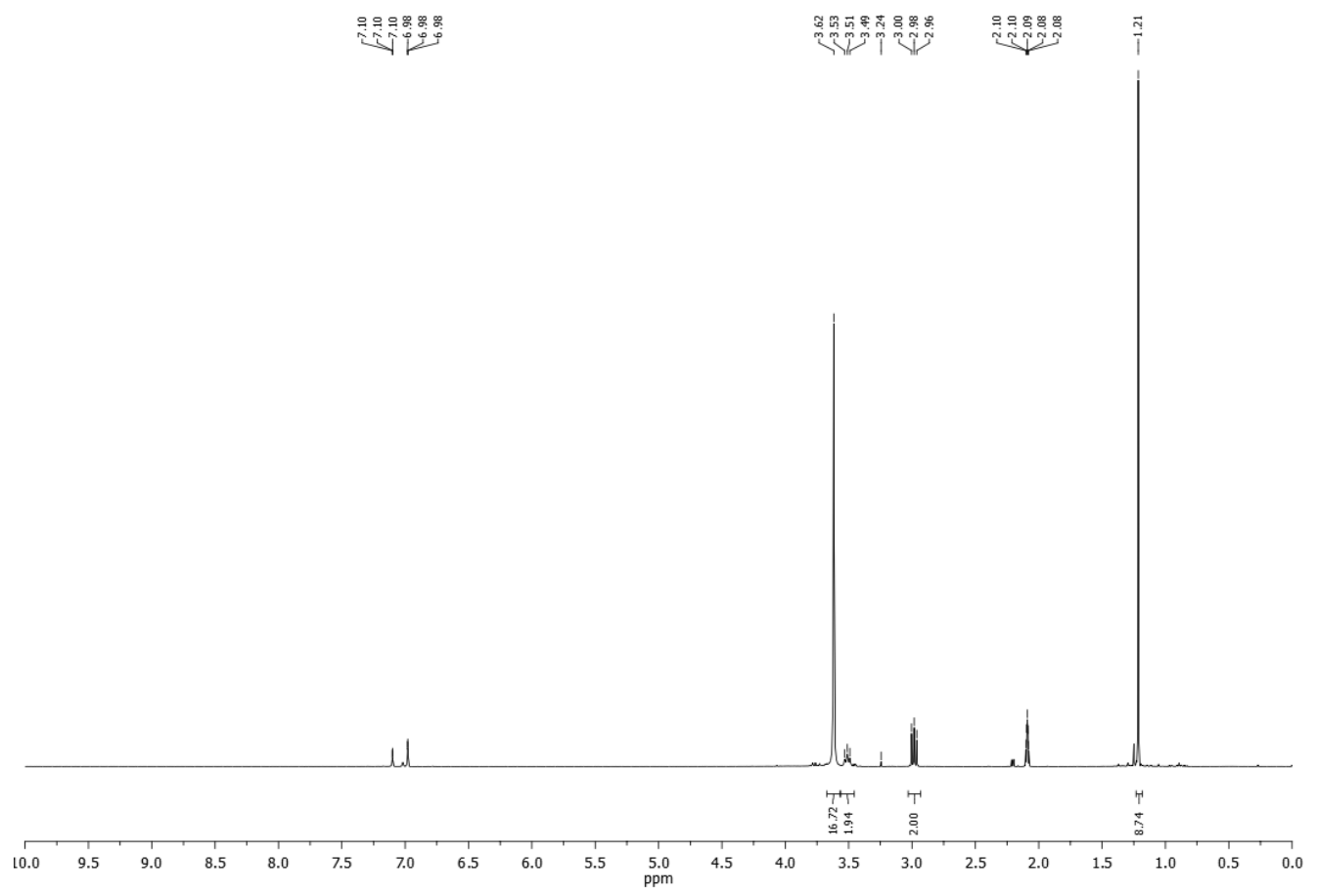

Figure S64. ${ }^{1} \mathrm{H}$ NMR ( $d_{8}$-toluene, $\left.400 \mathrm{MHz}, 298 \mathrm{~K}\right),\left[\mathrm{Ta}\left(\mathrm{NMe}_{2}\right)_{3} \mathrm{Cl}_{2}\right]_{2}+\mathrm{L} 2 \mathrm{Na}$ at $110{ }^{\circ} \mathrm{C}$ for $45 \mathrm{~min}$.

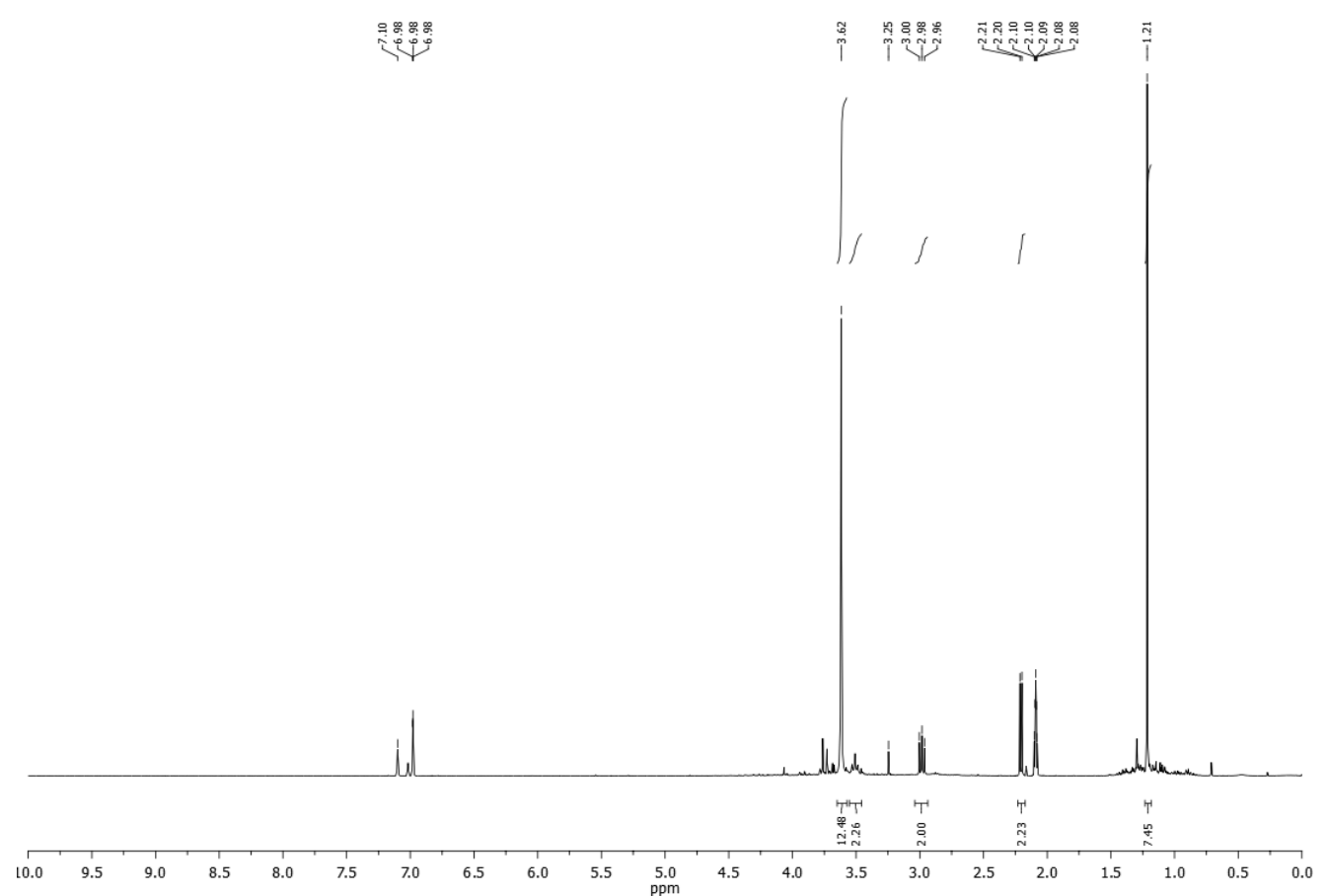

Figure S65. ${ }^{1} \mathrm{H}$ NMR $\left(d_{8}\right.$-toluene, $\left.400 \mathrm{MHz}, 298 \mathrm{~K}\right),\left[\mathrm{Ta}\left(\mathrm{NMe}_{2}\right)_{3} \mathrm{Cl}_{2}\right]_{2}+\mathrm{L} 2 \mathrm{Na}$ heated at $150{ }^{\circ} \mathrm{C}$ for $2 \mathrm{~h}$. 


\section{S5. Crystallographic details}

A summary of the crystallographic data for complexes 1, 2 and $\mathbf{2 4}$ is shown in Table S3. COSMO was used to determine the automatic data collection strategy and the cell determination and integration processes were done using SAINT. Using Olex2, ${ }^{[7]}$ the structures were solved with the ShelXT10 structure solution program using Intrinsic Phasing and the structures were refined using the ShelXL11 refinement package using the Least Squares method.

\section{S6. Crystallographic table:}

Table S3. List of crystallographic parameters for compounds 1, 2 and 24.

\begin{tabular}{|c|c|c|c|c|}
\hline \multicolumn{2}{|c|}{ Compound } & 1 & 2 & 24 \\
\hline \multicolumn{2}{|c|}{ Formula } & $\mathrm{C}_{19} \mathrm{H}_{46} \mathrm{CIN}_{2} \mathrm{OS} \mathrm{Si}_{3} \mathrm{Ta}$ & $\mathrm{C}_{28} \mathrm{H}_{55} \mathrm{CIN}_{5} \mathrm{OTa}$ & $\mathrm{C}_{16} \mathrm{H}_{37} \mathrm{CIN}_{5} \mathrm{OTa}$ \\
\hline \multicolumn{2}{|c|}{$M_{w}(\mathrm{~g} / \mathrm{mol}) ; d_{\text {calcd. }}\left(\mathrm{g} / \mathrm{cm}^{3}\right)$} & $619.25 ; 1.467$ & $694.17 ; 1.496$ & $531.90 ; 1.557$ \\
\hline \multicolumn{2}{|c|}{$T(K) ; F(000)$} & $90 ; 1256$ & $90 ; 1424$ & $90 ; 532$ \\
\hline \multicolumn{2}{|c|}{ Crystal System } & Monoclinic & Orthorhombic & Monoclinic \\
\hline \multicolumn{2}{|c|}{ Space Group } & $P\left(2_{1}\right) / n$ & $P 2{ }_{1} 2_{1} 2_{1}$ & $P 2_{1}$ \\
\hline \multirow[t]{6}{*}{ Unit Cell: } & $a(\AA)$ & $13.6655(7)$ & $11.4710(4)$ & $9.9984(6)$ \\
\hline & $b(\AA)$ & $12.2886(6)$ & $11.9421(4)$ & $8.1066(5)$ \\
\hline & $c(\AA)$ & $16.7490(8)$ & $22.4946(8)$ & $14.0375(9)$ \\
\hline & $\alpha\left({ }^{\circ}\right)$ & 90 & 90 & 90 \\
\hline & $\beta\left(^{\circ}\right)$ & $94.6700(10)$ & 90 & $94.491(3)$ \\
\hline & $Y\left({ }^{\circ}\right)$ & 90 & 90 & 90 \\
\hline \multicolumn{2}{|c|}{$V\left(\AA^{3}\right) ; Z$} & 2803.3(2); 4 & $3081.49(18) ; 4$ & $1134.29(12) ; 2$ \\
\hline \multicolumn{2}{|c|}{$\mu\left(\mathrm{mm}^{-1}\right)$; Abs. Corr. } & 4.156; multiscan & 3.682; multiscan & 4.975; multiscan \\
\hline \multicolumn{2}{|c|}{$\theta$ range $\left({ }^{\circ}\right)$; completeness } & $2.44-32.01 ; 1$ & 2.46-29.56; 1 & $2.42-30.09 ; 1$ \\
\hline \multicolumn{2}{|c|}{ collected reflections; $\mathbf{R}_{\boldsymbol{\sigma}}$} & $41312 ; 0.0332$ & $21575 ; 0.0905$ & $24148 ; 0.0268$ \\
\hline \multicolumn{2}{|c|}{ unique reflections; $\mathbf{R}_{\mathrm{int}}$} & $9738 ; 0.0381$ & $8638 ; 0.0597$ & $6631 ; 0.0310$ \\
\hline \multicolumn{2}{|c|}{$\mathrm{R} 1(\mathrm{~F})(\mathrm{I}>2 \sigma(\mathrm{I}))$} & 0.0204 & 0.0394 & 0.0322 \\
\hline \multicolumn{2}{|c|}{$w R\left(F^{2}\right)$ (all data) } & 0.0436 & 0.0633 & 0.0825 \\
\hline \multicolumn{2}{|c|}{ GoF $\left(F^{2}\right) ;$ Flack-x } & 1.023; - & $0.945 ; 0.092(11)$ & $1.126 ;-0.013(12)$ \\
\hline \multicolumn{2}{|c|}{ Residual electron density } & $0.961 ;-0.598$ & $1.188 ;-0.873$ & $0.994 ;-1.268$ \\
\hline
\end{tabular}


Figure S66. ORTEP representation of the molecular structure of 1. The hydrogen atoms are omitted for clarity. Ellipsoids are drawn at the $50 \%$ probability level. Selected bond lengths: Ta1-Cl1, 2.3898(4) $\AA$; Ta1-O1, 2.3240(12) A; Ta1-N2, 2.0694 (15) Å; Ta1-C8, 2.1821(16) Å; Ta1-C12, 2.1501(16) Å; Ta1-C16, 2.1553(18) Å. Selected bond angles: O1-Ta1-N2, 60.15(5) ${ }^{\circ}$; O1-Ta1-Cl1, 137.76(3) ${ }^{\circ}$ N N2-Ta1-Cl1, 161.93(4) .

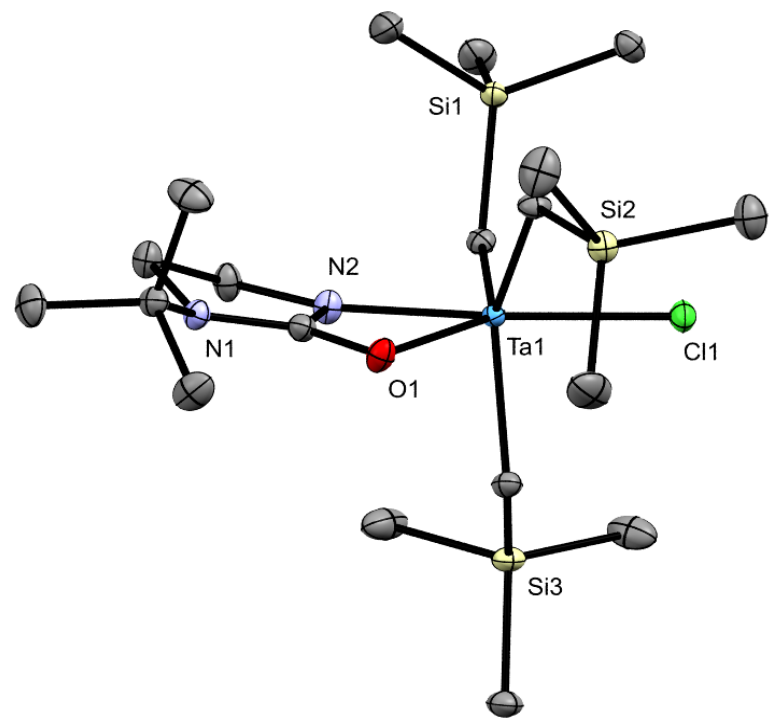

Figure S67. ORTEP representation of the molecular structure of 2. The hydrogen atoms are omitted for clarity. Ellipsoids are drawn at the $50 \%$ probability level. Selected bond lengths: Ta1-Cl1, 2.5505(15) $\AA$; Ta1-O1, 2.230(4) $\AA$; Ta1-N2,

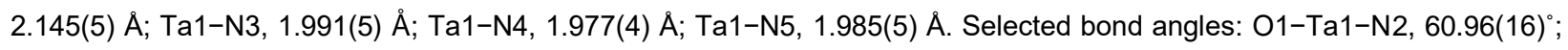
O1-Ta1-Cl1, 82.68(13) ${ }^{\circ}$ N2-Ta1-Cl1, 81.48(18) ${ }^{\circ}$.

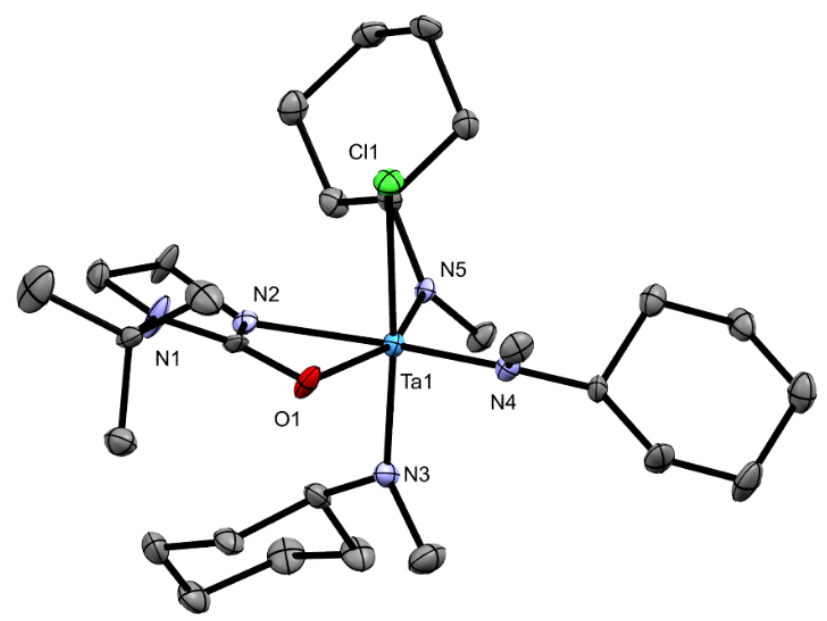


Figure S68. ORTEP representation of the molecular structure of $\mathbf{2 4}$. The hydrogen atoms are omitted for clarity. Ellipsoids are drawn at the $50 \%$ probability level. Selected bond lengths: Ta1-Cl1, 2.5165(13) A; Ta1-O1, 2.244(4) Å; Ta1-N2, 2.160(4) Å; Ta1-N3, 1.994(5) Å; Ta1-N4, 1.990(5) Å; Ta1-N5, 1.977(4) Å. Selected bond angles: O1-Ta1-N2, 61.26(15) ; O1-Ta1-Cl1, 84.05(11) ; N2-Ta1-Cl1, 82.00(12) .

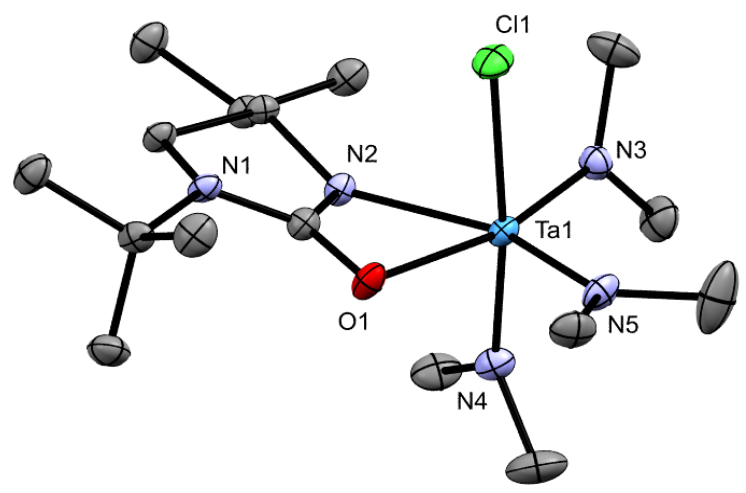

\section{References:}

[1] J. W. Brandt, E. Chong, L. L. Schafer, ACS Catalysis 2017, 7, 6323-6330.

[2] G. Zweifel, H. P. On, Synthesis 1980, 1980, 803-805.

[3] aR. C. DiPucchio, S.-C. Roşca, L. L. Schafer, Angewandte Chemie International Edition 2018, 57, 3469-3472; bS. Moorhouse, G. Wilkinson, Journal of the Chemical Society, Dalton Transactions 1974, 2187-2190.

[4] G. L. Khatik, V. Kumar, V. A. Nair, Organic Letters 2012, 14, 2442-2445.

[5] P. R. Payne, J. A. Bexrud, D. C. Leitch, L. L. Schafer, Canadian Journal of Chemistry 2011, 89, 1222-1229.

[6] X. Qi, R. Zhou, J.-B. Peng, J. Ying, X.-F. Wu, European Journal of Organic Chemistry 2019, 2019, 5161-5164.

[7] O. V. Dolomanov, L. J. Bourhis, R. J. Gildea, J. A. K. Howard, H. Puschmann, Journal of Applied Crystallography 2009, 42, 339-341. 